\title{
Fixing All Moduli in a Simple F-theory Compactification
}

\section{Citation}

Denef, Frederik, Michael R. Douglas, Bogdan Florea, Antonella Grassi, and Shamit Kachru. 2005. Fixing all moduli in a simple F-theory compactification. Advances in Theoretical and Mathematical Physics 9(6): 861-929.

\section{Published Version}

http://www.intlpress.com/ATMP/ATMP-issue_9_6.php

\section{Permanent link}

http://nrs.harvard.edu/urn-3:HUL.InstRepos:3138322

\section{Terms of Use}

This article was downloaded from Harvard University's DASH repository, and is made available under the terms and conditions applicable to Other Posted Material, as set forth at http:// nrs.harvard.edu/urn-3:HUL.InstRepos:dash.current.terms-of-use\#LAA

\section{Share Your Story}

The Harvard community has made this article openly available.

Please share how this access benefits you. Submit a story.

Accessibility 
hep-th/0503124

SLAC-PUB-11015

SU-ITP-05/07

\title{
Fixing All Moduli in a Simple F-Theory Compactification
}

\author{
Frederik Denef ${ }^{\natural 1}$, Michael R. Douglas ${ }^{b 1, \&}$, Bogdan Florea ${ }^{\sharp 1}$, Antonella Grassi ${ }^{\exists 2}$ and Shamit Kachru $^{\forall 3}$ \\ ${ }^{1}$ Department of Physics and Astronomy, Rutgers University, \\ Piscataway, NJ 08855-0849, USA \\ ${ }^{\&}$ I.H.E.S., Le Bois-Marie, Bures-sur-Yvette, 91440 France \\ ${ }^{2}$ Department of Mathematics, University of Pennsylvania, \\ Philadelphia, PA 19104-6395, USA \\ ${ }^{3}$ Department of Physics and SLAC, Stanford University, \\ Stanford, California, CA 94305, USA
}

We discuss a simple example of an F-theory compactification on a Calabi-Yau fourfold where background fluxes, nonperturbative effects from Euclidean D3 instantons and gauge dynamics on D7 branes allow us to fix all closed and open string moduli. We explicitly check that the known higher order corrections to the potential, which we neglect in our leading approximation, only shift the results by a small amount. In our exploration of the model, we encounter interesting new phenomena, including examples of transitions where D7 branes absorb O3 planes, while changing topology to preserve the net D3 charge.

March 2005

\footnotetext{
औ denef@physics.rutgers.edu

b mrd@physics.rutgers.edu

\# florea@physics.rutgers.edu

$\exists$ grassi@math.upenn.edu

$\forall$ skachru@stanford.edu
} 


\section{Introduction}

All well-studied quasi-realistic string compactifications come, in the leading approximation, with large numbers of scalar moduli fields. It has long been thought that this is an artifact of the leading approximation, and that in many backgrounds non-perturbative effects generate potentials which lift all of these fields.

More recently, it has been emphasized that such potentials can have a very large number of minima, which combined with other choices made in the constructions gives rise to an immense 'landscape' of string vacua [22, 77, 1, 65, 96, 43]. Indeed, very simple genericity arguments, based on knowledge of effective field theory and of the contributions of various stringy ingredients to the effective potential, suggest that this should be true.

Since typical constructions have dozens or hundreds of moduli fields, and many different effects contributing to the scalar potential, the explicit calculations required to verify this expectation are daunting in almost all examples. However it is important to pursue explicit examples in as much detail as possible. Such examples allow one both to verify the hypothesis that there are many models with stabilized moduli, and to gain further intuition about stringy potentials which may be relevant in developing models of inflation or particle physics.

A proposal for stabilizing a wide class of type IIB or F-theory compactifications was put forth in [65], and fairly explicit examples were provided in [37]. In F-theory on an elliptic Calabi-Yau fourfold, the moduli of interest are the complex structure moduli and the Kähler moduli (which come from $h^{1,1}$ of the base of the elliptic fibration). In the limit where one can think of these constructions in terms of IIB Calabi-Yau orientifolds, these moduli include the moduli of the Calabi-Yau threefold, the axio-dilaton, and the positions of D7-branes in the threefold geometry.

In the constructions described in [65], one approaches the problem of moduli stabilization in two steps. First, one turns on background fluxes to stabilize the complex structure moduli of the fourfold at some energy scale $E$ - the fluxes can in general stabilize both the complex structure and D7 brane moduli, from the IIB perspective. Then, one incorporates exponentially small effects arising from Euclidean D3 instantons (corresponding to M5 instantons wrapping vertical divisors of holomorphic Euler characteristid $1 \chi_{h}=1$ ) or infrared gauge dynamics on D7 branes, to generate a potential for the Kähler moduli

1 This is often (but not quite accurately) called the arithmetic genus in the physics literature in this context. 
at the scale $\tilde{E}$. At any moderately large radius in string units, one finds $E>>\tilde{E}$, and it is a good approximation to treat the complex moduli as fixed when one evaluates the effects generating a potential for the Kähler modes (so any complex structure-dependent determinants multiplying the instanton action, should be evaluated at the critical point of the flux potential). As described in [65], as long as the gravitino mass $e^{K / 2}|W|$ resulting from the first step is moderately small in string units, one has a small parameter to use in the next step, which can result in radii stabilized within the regime of convergence of the instanton expansion. In fact, as we shall see explicitly, even $\mathcal{O}(1)$ values of $e^{K / 2}|W|$ can suffice in some concrete models.

In this paper, we provide an explicit example where this recipe is carried out in complete detail. We construct an orientifold of a Calabi-Yau threefold and its F-theory dual fourfold, which contains enough rigid $\chi_{h}=1$ divisors and pure non-abelian D7 brane gauge theories, to generate a potential for all Kähler modes. The Calabi-Yau threefold is the resolved orbifold $T^{6} / \mathbb{Z}_{2} \times \mathbb{Z}_{2}$, which has 51 Kähler moduli and 3 complex structure moduli. It is not difficult to construct explicit flux vacua in the complex structure sector of this model, and we provide some examples with moderately small $e^{K}|W|^{2}$. We solve for the stabilized values of all 51 Kähler moduli in the leading approximation, and show that the subleading corrections which we neglected are indeed expected to be quite small. Our example was chosen for its simplicity, especially as regards its complex structure moduli space (since we wished to provide explicit flux vacua), and hence it admits far fewer flux vacua than more generic fourfolds - only $\mathcal{O}\left(10^{13}\right)$, in contrast to the more impressive numbers like $\mathcal{O}\left(10^{300}\right)$ that arise in more complicated examples. In suitable cases with larger numbers of flux vacua, one expects similar constructions to be even more controlled, but of course the higher-dimensional complex structure moduli space becomes harder to work with explicitly.

The organization of this paper is as follows. In $\S 2$, we give a first description of our main model. We find that the Kähler moduli space has a rather intricate structure, with different phases that meet in a singular orbifold geometry. We argue that each blow-up mode of the orbifold which contributes a twisted Kähler mode in the $4 D$ theory, comes along with its own vertical divisor of holomorphic Euler characteristic one, and that the gauge theory sector is pure $S O(8)^{12} \mathcal{N}=1$ SYM without any matter. In $\S 3$, we describe the geometry of the F/M-theory dual fourfold in great detail, and put the arguments of $\S 2$ on firm mathematical footing. In $\S 4$, we discuss the relation of our model to some similar toroidal orientifolds which have (in absence of flux) exactly soluble worldsheet definitions at 
the orbifold point. In $\S 5$, we construct explicit flux vacua in this example, which stabilize the complex structure moduli and provide the needed moderately small $e^{K}|W|^{2}$. We choose to saturate the entire D3-tadpole by turning on fluxes, so no wandering D3 branes are introduced in the geometry. In $\S 6$, we then include the leading non-perturbative effects, and see that once one includes both the D3-instanton effects and the gauge dynamics on the D7 branes in this model, all untwisted and twisted Kähler moduli are stabilized. We check the magnitude of the known corrections to the leading result, from perturbative [10] and worldsheet instanton corrections to the Kähler potential as well as multi-instanton contributions to the superpotential, and see that even with our only moderately small $e^{K}|W|^{2}$ these corrections are expected to be at the one percent level. In the penultimate section, we briefly describe some other explicit models with only a single Kähler mode which are good candidates for stabilization. We conclude in $\S 8$. In the appendix, we describe our normalization conventions.

\section{Orientifold Geometry}

Our model is a type IIB orientifold of a smooth Calabi-Yau threefold $Y$, which is a resolution of the orbifold $\bar{Y}=T^{6} / \mathbb{Z}_{2} \times \mathbb{Z}_{2}$. The orbifold group acts as

$\begin{array}{cccc} & z_{1} & z_{2} & z_{3} \\ \alpha & + & - & - \\ \beta & - & + & - \\ \alpha \circ \beta & - & - & +\end{array}$

There are $3 \times 16=48$ fixed lines under the action of a group element, and 64 fixed points where three fixed lines meet. Blowing up the fixed lines resolves the geometry and introduces 48 twisted Kähler moduli in addition to the 3 untwisted Kähler moduli descending from $\left(T^{2}\right)^{3}$. There are just 3 complex structure moduli, given by the modular parameters of the $T^{2}$ factors. Thus the resulting smooth Calabi-Yau threefold $Y$ has $h^{1,1}=51, h^{2,1}=3$. This is one of the Borcea-Voisin models [21,100].

We will see in the following that we can choose an orientifold involution of $Y$ and D7-brane embeddings in such way that we get an $S O(8)^{12}$ gauge group without matter, and a D3 tadpole $Q_{3}=-28$ (on the quotient space) induced by non-exotic O3-planes and 7-brane curvature. 
Before proceeding, let us briefly explain why we do not define our model as a toroidal orientifold, i.e. by orientifolding the orbifold $\bar{Y}$. Despite the geometric singularities, orientifolds of an orbifold can often be described directly by perturbative string CFT's, and the case of $T^{6} / \mathbb{Z}_{2} \times \mathbb{Z}_{2}$ has been studied extensively.

At first sight, the toroidal orientifold appears promising: the involution $\Omega: z_{i} \rightarrow-z_{i}$, which (modulo the $\mathbb{Z}_{2} \times \mathbb{Z}_{2}$ equivalences) has $3 \times 4=12 \mathrm{O} 7$ fixed planes and 64 fixed points at the triple intersections of the O7 planes, each of which corresponds to an O3 plane, roughly matching the smooth geometry we are about to describe. This comparison will be carried much further in $\S 4$, where it is shown that the $S O(8)^{12}$ gauge group and many other features of our model can be realized by a toroidal orientifold.

However, in general, not every large radius orientifold model has an orbifold limit with a well-behaved CFT description. When trying to go continuously from large radii to the orbifold point, nonperturbative massless states could arise from D-branes wrapping vanishing cycles, rendering perturbation theory singular. In the parent $\mathcal{N}=2$ theories, such singularities are of complex codimension one in moduli space, so one can go around them and continuously connect various phases. However, after orientifolding, these singularities become of real codimension 1, preventing different phases from being connected smoothly. Therefore, orientifolds constructed geometrically in the large radius regime do not necessarily all have orbifold CFT counterparts, and in particular may have different discrete properties.

Indeed, we will see that this is the case for our model in $\S 4$. For this reason, and because we will stabilize all radii at finite distance away from the orbifold point anyway, we will first blow up the orbifold into a smooth, large Calabi-Yau threefold $Y$, using standard techniques in algebraic geometry. We then consider the string theory whose world-sheet definition is the sigma model with this Calabi-Yau target space, and then define an orientifold of this model. The result is similar to a toroidal orientifold but realizes discrete choices not possible in the CFT orientifold framework. Still, readers familiar with that framework may find it useful to read $\S 4$ to get an overview of the construction before proceeding.

In the following, we will first study a local description valid near the orbifold fixed points. We give a completely explicit description of the resolution, the orientifold involution, and the brane embeddings. We also review how intersection numbers, important for example to derive the Kähler potential, are computed in this setup. We then move on to the compact Calabi-Yau and discuss its lift to F-theory on an elliptically fibered 
Calabi-Yau fourfold (which we define as M-theory in the limit of vanishing elliptic fiber area). Finally, we calculate the D3 tadpole for our model, and note an interesting geometrical transition where a 7-brane stack "eats" an O3-plane while changing its topology to preserve the net D3-brane charge.

\subsection{Local model}

To understand the resolved geometry and the orientifold involution, it is useful to consider first a local model of the singularities, given by $\bar{X}=\mathbb{C}^{3} / \mathbb{Z}_{2} \times \mathbb{Z}_{2}$. The resolution of this orbifold can be described explicitly as a toric variety, following the general construction outlined in [34].

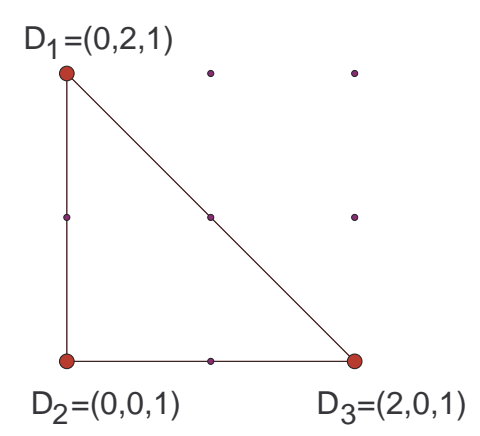

Fig. 1: Top view of fan of $\mathbb{C}^{3} / \mathbb{Z}_{2} \times \mathbb{Z}_{2}$ (unresolved).

The data underlying any three dimensional toric variety is given by a lattice $N=\mathbb{Z}^{3}$ and the choice of a fan $\Delta$, which is a collection of cones generated by lattice points in $N$, satisfying the condition that every face of a cone is also a cone, and that the intersection of two cones is a face of each.

The singular orbifold $\mathbb{C}^{3} / \mathbb{Z}_{2} \times \mathbb{Z}_{2}$ is described by the simple fan given in fig. 1 , consisting of a single 3 -dimensional cone generated by the lattice vectors $D_{1}=(0,2,1)$, $D_{2}=(0,0,1)$ and $D_{3}=(2,0,1)$. As usual for Calabi-Yau varieties, the third component of each vector equals 1 , so we can restrict our attention to the other two coordinates, as we did in the figure. To each vertex $D_{i}$ a complex variable $z_{i}$ is assigned, and to each dimension $r=1, \ldots, 3$ a monomial $U_{r} \equiv \prod_{i} z_{i}^{\left(D_{i}\right)_{r}}$. In this case, $U_{1}=z_{3}^{2}, U_{2}=z_{1}^{2}$, $U_{3}=z_{1} z_{2} z_{3}$. The toric variety $\bar{X}$ is then simply given by all $\left(z_{1}, z_{2}, z_{3}\right)$ not in a certain set $F$, modulo complex rescalings that leave the $U_{r}$ invariant. The excluded set $F$ is given by the values of $z_{i}$ which have simultaneous zeros of coordinates not belonging to the same cone. Since there is only one three dimensional cone here, $F$ is empty. The only rescalings that leave the $U_{r}$ invariant are given precisely by (2.1). Thus, $\bar{X}$ is indeed $\mathbb{C}^{3} / \mathbb{Z}_{2} \times \mathbb{Z}_{2}$. 


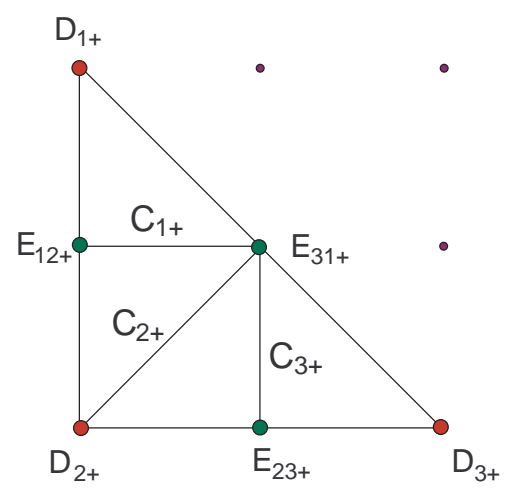

(a)

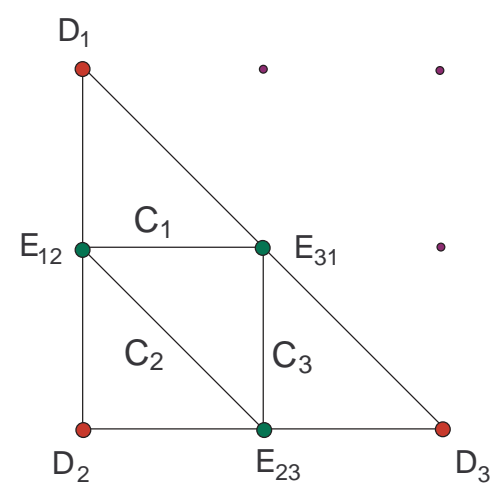

(b)

Fig. 2: Fan for $(a)$ asymmetric and (b) symmetric resolution of $\mathbb{C}^{3} / \mathbb{Z}_{2} \times \mathbb{Z}_{2}$.

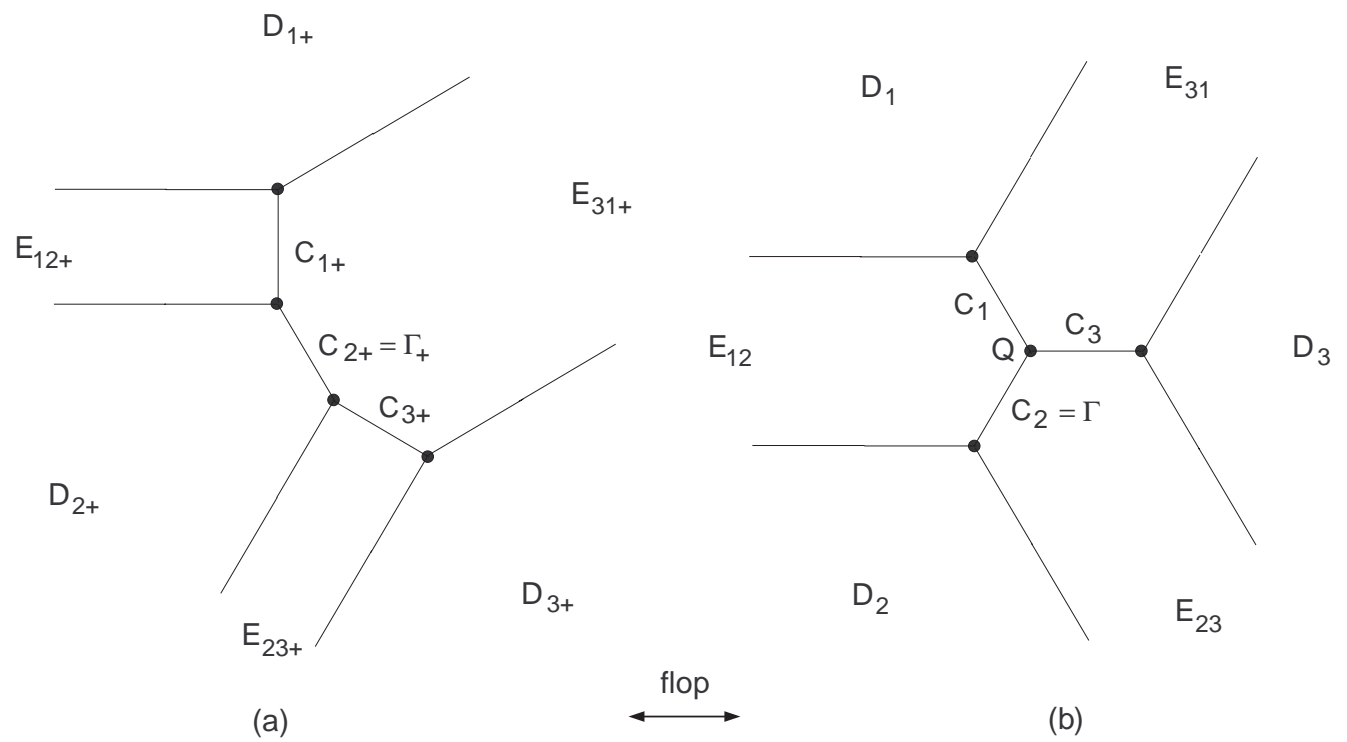

Fig. 3: Dual graph for $(a)$ asymmetric and (b) symmetric resolution.

The fact that $\bar{X}$ is singular can be traced back to the fact that the top dimensional cone generators do not span the full lattice $N$, since $\operatorname{det}\left(D_{i, r}\right)=4$. To resolve the variety, one has to refine the fan such that all top dimensional cones have determinant 1 . There are two distinct ways of doing this in the case at hand, one symmetric and one asymmetric, as shown in fig. $2(b)$ resp. (a). The dual graphs are shown in fig. 3. As we will review below, the vertices in fig. 2 can be thought of as divisors, the lines as curves at the intersections of two divisors, and the faces as points at the intersections of three divisors. In the dual graphs, the role of faces and vertices is interchanged. 


\section{Symmetric resolution}

As shown in fig. $2(b)$, there are now 6 vertices and 4 cones $\left(D_{1}, E_{12}, E_{31}\right)$, $\left(D_{2}, E_{12}, E_{2,3}\right),\left(D_{3}, E_{23}, E_{3,1}\right),\left(E_{12}, E_{23}, E_{31}\right)$ all of determinant 1 . The vertices are given by the matrix

$\begin{array}{cccccc}D_{1} & D_{2} & D_{3} & E_{23} & E_{31} & E_{12} \\ 0 & 0 & 2 & 1 & 1 & 0 \\ 2 & 0 & 0 & 0 & 1 & 1 \\ 1 & 1 & 1 & 1 & 1 & 1\end{array}$.

We associate complex variables $z_{i}$ to the $D_{i}$ and $y_{i}$ to the $E_{j k}$. The powers in the monomials $U_{r}$ are simply given by the rows of this matrix, i.e. $U_{1}=z_{3}^{2} y_{1} y_{2}, U_{2}=z_{1}^{2} y_{2} y_{3}, U_{3}=$ $z_{1} z_{2} z_{3} y_{1} y_{2} y_{3}$. The rescalings leaving the $U_{r}$ invariant are

$$
\left(z_{1}, z_{2}, z_{3}, y_{1}, y_{2}, y_{3}\right) \rightarrow\left(\lambda_{1} z_{1}, \lambda_{2} z_{2}, \lambda_{3} z_{3}, \frac{\lambda_{1}}{\lambda_{2} \lambda_{3}} y_{1}, \frac{\lambda_{2}}{\lambda_{3} \lambda_{1}} y_{2}, \frac{\lambda_{3}}{\lambda_{1} \lambda_{2}} y_{3}\right)
$$

with $\lambda_{i} \in \mathbb{C}^{*}$. The set of excluded points $F$ is again given by simultaneous zeros of coordinates not in the same cone, for example $\left(z_{1}, y_{1}\right)=(0,0)$ and $\left(z_{2}, y_{1}, z_{3}\right)=(0,0,0)$ are excluded, but $\left(z_{2}, y_{1}\right)=(0,0)$ is not. The toric variety is thus given by $X=\left(\mathbb{C}^{6} \backslash F\right) / \mathbb{C}^{* 3}$, with the $\mathbb{C}^{*}$ actions given in (2.3).

To each vertex corresponds a toric divisor, by setting the associated coordinate equal to zero. Curves are obtained by intersecting divisors, i.e. setting two coordinates to zero. To avoid being on the excluded locus $F$, the corresponding vertices must be part of the same cone, in other words they have to be joined by a line in fig. 2. Compact curves correspond to internal lines. In the case at hand, there are three such curves, which we denote by $C_{i}$, where $C_{1}=E_{31} \cdot E_{12}$ and cyclic permutations thereof. Finally, triple intersection points of divisors are obtained by setting the 3 coordinates associated to a single cone to zero. Thus the triple intersection number is 1 for 3 distinct divisors belonging to the same cone, and 0 otherwise.

The divisors $D_{i}$ are the original divisors we had in the unresolved variety, the $E_{i j}$ are the exceptional divisors produced by the resolution, and the $C_{i}$ are the exceptional curves. The latter have topology $\mathbb{P}^{1}$. This can be seen as follows. $C_{1}$ for example is given by $y_{2}=y_{3}=0$. To avoid the excluded set $F$, we must take $z_{2} \neq 0, z_{3} \neq 0$ and $\left(z_{1}, y_{1}\right) \neq(0,0)$. This allows choosing a gauge with $z_{2}=z_{3}=1$, so

$$
C_{1}=\left\{\left(z_{1}, 1,1, y_{1}, 0,0\right) \mid\left(z_{1}, y_{1}\right) \in \mathbb{C}^{2} \backslash(0,0)\right\} /\left(z_{1}, y_{1}\right) \sim \lambda\left(z_{1}, y_{1}\right)
$$

which is of course $\mathbb{P}^{1}$. 


\section{Orientifold action and D-brane embedding}

Let us now look at the orientifold involution. There are several choices. We choose $\Omega:(z, y) \rightarrow(-z, y)$. The fixed points are then given by the $(z, y)$ for which

$$
(-z, y)=\left(\lambda_{1} z_{1}, \lambda_{2} z_{2}, \lambda_{3} z_{3}, \frac{\lambda_{1}}{\lambda_{2} \lambda_{3}} y_{1}, \frac{\lambda_{2}}{\lambda_{3} \lambda_{1}} y_{2}, \frac{\lambda_{3}}{\lambda_{1} \lambda_{2}} y_{3}\right)
$$

for some $\lambda_{i}$. The following possibilities arise:

(1) If all $z_{i} \neq 0$, we need $\lambda_{i}=-1$ and therefore $y=-y=0$. Because $\left(y_{1}, y_{2}, y_{3}\right)$ belong to the same cone, this is an allowed point. Thus, we get an isolated fixed point $Q$ that can be represented by $(1,1,1,0,0,0)$. This corresponds to an O3-plane.

(2) If say $z_{1}=0$, then to avoid the excluded set $F$, we must take $z_{2} \neq 0, z_{3} \neq 0$, $y_{1} \neq 0$. Then (2.5) implies $\lambda_{2}=\lambda_{3}=-1$ and $\lambda_{1}=1$, and imposes no further constraints. Therefore the entire divisor $D_{1}: z_{1}=0$ is fixed. This gives us an O7-plane. Similarly, there will be $\mathrm{O} 7$ planes on $D_{2}$ and $D_{3}$. The topology of these divisors is easily determined. Since $z_{2}, z_{3}$ and $y_{1}$ are all nonzero, we can fix the scaling gauge by setting these variables equal to 1 . The divisor is then parametrized by the remaining variables $y_{2}$ and $y_{3}$ without further identifications, so it is a copy of $\mathbb{C}^{2}$.

The action of $\Omega$ on the exceptional $\mathbb{P}^{1} \mathrm{~s}$ is also straightforward to determine. After a gauge transformation $\lambda_{i}=-1$, the orientifold action can be written as $(z, y) \rightarrow(z,-y)$, which acts on (2.4) as

$$
\left[z_{1}, y_{1}\right] \rightarrow\left[z_{1},-y_{1}\right]
$$

That is, the $\mathbb{P}^{1} \mathrm{~s}$ are mapped to themselves in an orientation preserving way, with fixed points at the poles, where the $\mathbb{P}^{1}$ intersects the $\mathrm{O} 3$ or $\mathrm{O} 7$ planes. Note that one cannot wrap a closed string once around a pole of the quotient $\mathbb{P}^{1} / \mathbb{Z}_{2}$, since the endpoints of a string can only be identified by an orientifold $\mathbb{Z}_{2}$ if the orientation of the string is reversed. Therefore the minimal closed string instanton wraps $\mathbb{P}^{1} / \mathbb{Z}_{2}$ twice (or the original $\mathbb{P}^{1}$ once). The instanton phase should furthermore be invariant under the orientifold action $B \rightarrow-B$, which implies

$$
e^{2 \pi i \int_{\mathbb{P}^{1}} B}= \pm 1
$$

We still have to specify how we embed D-branes in this geometry. We will put D7branes on top of the O7-planes such that D7 tadpole is canceled locally. We choose the O7-planes to be non-exotic, so each induces -8 units of D7-brane charge in the Calabi-Yau $X$ (or -4 in the quotient $X / \mathbb{Z}_{2}$ ), and we need a stack of 8 coincident D7-branes on each 
$D_{i} \subset X$ to cancel this. This gives rise to an $S O(8)$ gauge group on each divisor $D_{i}$. Note that since the $D_{i}$ are disjoint in the resolved manifold, there are no massless bifundamentals from strings stretching between the D7-branes. To decide if there is massless adjoint matter, we need to know the topology of the $D_{i}$ in the compact geometry. We will get to this further on.

\section{Intersection numbers}

To construct the Kähler potential on moduli space, we will need the triple intersection numbers of the divisors, including triple intersections involving identical divisors. These numbers also determine self-intersections of curves inside divisors, which characterize the local geometry. In this subsection we will review how to obtain these numbers. The reader who is only interested in the results can safely skip this part however.

We can derive the intersection numbers in the local model for compact intersections. Linear combinations of divisors whose associated line bundle is trivial on the noncompact variety $X$ will give zero compact intersections with any combination of other divisors. Denoting the divisors collectively as $R_{a}, a=1, \ldots, 6$, and the corresponding coordinates by $x_{a}$, we have that $f=\prod_{a} x_{a}^{n_{a}}$ is a section of the line bundle $R=\sum n_{a} R_{a}$. The invariant monomials $U_{r}$ are functions, so the corresponding $R$ is trivial. For the purpose of computing compact intersections, this implies three linear relations between our divisors 2 : $2 D_{1}+E_{31}+E_{12}=0$, and so on. We should emphasize that this relation does not mean that this linear combination of divisors will also be trivial in the compact geometry. Rather, it means that this linear combination does not intersect the compact curves, and hence can be moved away from the origin - in the compact geometry, such a divisor corresponds to a "sliding divisor" such as $R_{1}:\left\{z_{1}=c\right\} \cup\left\{z_{1}=-c\right\}$. These divisors descend directly from the unresolved $T^{6} / \mathbb{Z}_{2} \times \mathbb{Z}_{2}$, and are in this sense independent of the blowup.

At any rate, these relations together with the triple intersection numbers of distinct divisors obtained directly from the fan are sufficient to determine all compact triple intersection numbers. This gives for example $E_{12}^{2} \cdot E_{23}=-\left(2 D_{1}+E_{31}\right) \cdot E_{12} \cdot E_{23}=-1$. From this, we also obtain the intersection numbers of the divisors with the compact curves

2 Despite the abuse of notation, these relations between divisors should not be confused with the relations between the corresponding vertices (2.2)! 
$C_{i}$ defined above. These curves form a basis of the Mori cone i.e. the cone of effective holomorphic curves in $X$. We get for their intersections:

$$
\begin{array}{ccccccc} 
& D_{1} & D_{2} & D_{3} & E_{23} & E_{31} & E_{12} \\
E_{31} \cdot E_{12} \equiv C_{1} & 1 & 0 & 0 & 1 & -1 & -1 \\
E_{12} \cdot E_{23} \equiv C_{2} & 0 & 1 & 0 & -1 & 1 & -1 \\
E_{23} \cdot E_{31} \equiv C_{3} & 0 & 0 & 1 & -1 & -1 & 1
\end{array}
$$

Note that the entries are precisely the charges of the rescalings (2.3). Indeed, the Mori cone intersection numbers always form a basis of the rescaling charges. This is an elementary algebraic consequence of the various definitions we made.

The triple intersection numbers also give the self-intersection numbers of the curves inside the exceptional divisors, for example $\left.C_{1}^{2}\right|_{E_{12}}=E_{12} \cdot E_{31}^{2}=-1$.

Finally, apart from one subtlety, it is straightforward to deduce the intersection numbers of the orientifold quotient $X / \mathbb{Z}_{2}$. The subtlety is the following. Denote the projection from $X$ to $X / \mathbb{Z}_{2}$ by $\pi$. Naively one might think one should take the toric divisors $\widetilde{R}_{a}$ of the quotient, considered as 2 -forms, to be related to those of the double cover by $R_{a}=\pi^{*} \widetilde{R}_{a}$. This is correct for the divisors $E_{i j}$, but not for the $D_{i}$, for which we should take $D_{i}=\pi^{*} \widetilde{D}_{i} / 2$. This can be seen as follows. Because $D_{i}$ is fixed by the $\mathbb{Z}_{2}$, its volume in $X$ must equal the volume of $\widetilde{D}_{i}$ in the quotient. But the volume of $\widetilde{D}_{i}$ is given by

$$
\int_{X / \mathbb{Z}_{2}} \widetilde{D}_{i} \wedge \frac{J \wedge J}{2}=\frac{1}{2} \int_{X} \pi^{*} \widetilde{D}_{i} \wedge \frac{J \wedge J}{2}
$$

which is half the volume of $\pi^{*} \widetilde{D}_{i}$. So we must take $D_{i}=\pi^{*} \widetilde{D}_{i} / 2$ to correct for this. For the divisors $E_{i j}$ on the other hand, whose volume does indeed get halved, there is no such correction factor of 2 . Thus we get for example

$$
\begin{aligned}
& \widetilde{D}_{1} \cdot \widetilde{E}_{31} \cdot \widetilde{E}_{12}=\frac{1}{2} \int_{X}\left(2 D_{1}\right) \wedge E_{12} \wedge E_{31}=D_{1} \cdot E_{12} \cdot E_{31}=1 \\
& \widetilde{E}_{12} \cdot \widetilde{E}_{23} \cdot \widetilde{E}_{31}=\frac{1}{2} \int_{X} E_{12} \wedge E_{23} \wedge E_{31}=\frac{1}{2} E_{12} \cdot E_{23} \cdot E_{31}=\frac{1}{2} .
\end{aligned}
$$

The half integral triple intersection product is possible because the intersection point coincides with the $\mathbb{Z}_{2}$ fixed point singularity $Q$ (the O3). For the intersections of the Mori cone generators, we thus get

$$
\begin{array}{lccccccc} 
& \widetilde{D}_{1} & \widetilde{D}_{2} & \widetilde{D}_{3} & \widetilde{E}_{23} & \widetilde{E}_{31} & \widetilde{E}_{12} \\
\widetilde{E}_{31} \cdot \widetilde{E}_{12} \equiv \widetilde{C}_{1} & 1 & 0 & 0 & 1 / 2 & -1 / 2 & -1 / 2 \\
\widetilde{E}_{12} \cdot \widetilde{E}_{23} \equiv \widetilde{C}_{2} & 0 & 1 & 0 & -1 / 2 & 1 / 2 & -1 / 2 \\
\widetilde{E}_{23} \cdot \widetilde{E}_{31} \equiv \widetilde{C}_{3} & 0 & 0 & 1 & -1 / 2 & -1 / 2 & 1 / 2
\end{array}
$$




\section{Asymmetric resolution}

The asymmetric resolution in fig. $2(a)$ can be treated in a completely analogous manner. The vertices of the fan remain the same, so the scalings (2.3) remain the same too. The cones themselves do change, so the excluded region $F$ will be different, as well as the intersection products. The generators of the Mori cone and their intersections are now given by 3

$\begin{array}{ccccccc} & D_{1} & D_{2} & D_{3} & E_{23} & E_{31} & E_{12} \\ E_{31} \cdot E_{12} \equiv C_{1} & 1 & 1 & 0 & 0 & 0 & -2 \\ D_{2} \cdot E_{31} \equiv C_{2} & 0 & -1 & 0 & 1 & -1 & 1 \\ E_{23} \cdot E_{31} \equiv C_{3} & 0 & 1 & 1 & -2 & 0 & 0\end{array}$

From this, we again get the self-intersections of the curves in the divisors: $\left.C_{1}^{2}\right|_{E_{12}}=$ $E_{31}^{2} \cdot E_{12}=0,\left.C_{1}^{2}\right|_{E_{31}}=-2,\left.C_{2}^{2}\right|_{E_{31}}=-1,\left.C_{2}^{2}\right|_{D_{2}}=-1$. At the level of the intersections, the curves $C_{i}$ are related to those of the symmetric resolution $C_{i}^{\prime}$ by $C_{1}^{\prime}=C_{1}+C_{2}, C_{2}^{\prime}=-C_{2}$, $C_{3}^{\prime}=C_{3}+C_{2}$. These relations are characteristic of a flop; indeed, the symmetric and asymmetric resolutions are related by flopping the curve $C_{2}$.

The orientifold action is again $\Omega:(z, y) \rightarrow(-z, y)$. As in the symmetric resolution, the divisors $D_{i}$ support O7-planes. Now however there is no isolated fixed point: $y=0$ lies in the excluded set $F$. All $\mathbb{P}^{1}$ 's are acted on by $\Omega$ as in (2.6), except $C_{2}$, which is pointwise fixed, since it is embedded in an O7-plane. The triple intersections of the quotient are obtained by the rules given earlier (i.e. add an overall factor of $1 / 2$ and $D_{i} \rightarrow 2 D_{i}$ ). This gives for the Mori cone

$$
\begin{array}{lcccccc} 
& \widetilde{D}_{1} & \widetilde{D}_{2} & \widetilde{D}_{3} & \widetilde{E}_{23} & \widetilde{E}_{31} & \widetilde{E}_{12} \\
\widetilde{E}_{31} \cdot \widetilde{E}_{12} \equiv \widetilde{C}_{1} & 1 & 1 & 0 & 0 & 0 & -1 \\
\widetilde{D}_{2} \cdot \widetilde{E}_{31} \equiv \widetilde{C}_{2} & 0 & -2 & 0 & 1 & -1 & 1 \\
\widetilde{E}_{23} \cdot \widetilde{E}_{31} \equiv \widetilde{C}_{3} & 0 & 1 & 1 & -1 & 0 & 0
\end{array}
$$

\subsection{Compact model}

To get the compact model $Y$, one simply glues the 64 local models together, with transition functions determined by the transition functions between the $z$-coordinates in the original $T^{6}$. This gives $3 \times 16=48$ exceptional divisors $E_{i \alpha, j \beta}$ and $3 \times 4=12$ O7-planes on divisors $D_{i \alpha}$. Here $i, j=1, \ldots, 3$ (with $i<j$ ) and $\alpha, \beta=1, \ldots, 4$. On each O7-plane, we furthermore put an $S O(8)$ stack of D7-branes. This locally cancels the D7-tadpole, so

3 To avoid cluttering, we drop the index ' + ' here. In section 3 , where the relation between the two resolutions will be studied in more detail, the ' + ' index will be reinstated. 
the axio-dilaton is constant on $Y$. In the symmetric resolution, there are 64 O3-planes. In the asymmetric resolution, these are absent.0

The global topology of the various divisors is easily deduced. Let us consider for example the divisors $D_{i \alpha}$ in the symmetric resolution. The topology of the resolved manifold with the exceptional divisors removed is the same as the topology of $T^{6} / \mathbb{Z}_{2} \times \mathbb{Z}_{2}$ with its singularities removed. In this space, the divisors $D_{i \alpha}$ have topology $T^{2} / \mathbb{Z}_{2} \times T^{2} / \mathbb{Z}_{2}$ with the singularities removed, that is $\mathbb{P}^{1} \times \mathbb{P}^{1}$ with four points removed in each $\mathbb{P}^{1}$ factor. In each local patch, this looks like $\mathbb{C} \times \mathbb{C}$ with the origin in each $\mathbb{C}$ factor removed. From the explicit construction of the local model given above, it is clear that in the resolved space, the origin of each $\mathbb{C}$ factor is simply put back as a point (as opposed to being replaced by some exceptional curve). Therefore, in the resolved compact model, the divisors $D_{i \alpha}$ are simply $\mathbb{P}^{1} \times \mathbb{P}^{1}$.

For the topology of the exceptional divisors $E_{i \alpha, j \beta}$ we get similarly $\mathbb{P}^{1} \times \mathbb{P}^{1}$ blown up in 4 points (corresponding to the four intersections of a fixed line with the fixed planes in $\left.T^{6} / \mathbb{Z}_{2} \times \mathbb{Z}_{2}\right)$. In the asymmetric resolution on the other hand the $D_{1}$ and $D_{3}$ divisors still have topology $\mathbb{P}^{1} \times \mathbb{P}^{1}$, but the $D_{2}$ divisors are now $\mathbb{P}^{1} \times \mathbb{P}^{1}$ blown up in 16 points. The $E_{12}$ and $E_{23}$ divisors are $\mathbb{P}^{1} \times \mathbb{P}^{1}$, and $E_{31}$ is $\mathbb{P}^{1} \times \mathbb{P}^{1}$ blown up in 8 points.

All these divisors evidently have $h^{1,0}=h^{2,0}=0$, since $\mathbb{P}^{1} \times \mathbb{P}^{1}$ has this property, and blowing up only changes $h^{1,1}$. This has important consequences:

(1) There is no massless adjoint matter in the $S O(8)^{12}$ gauge theory. Since moreover the $D_{i \alpha}$ do not intersect, there is no massless bifundamental matter either. So the gauge theory is pure $\mathcal{N}=1$ super Yang-Mills, and in particular will give rise to gaugino condensation and the generation of a nonperturbative superpotential for the Kähler moduli governing the size of the $D_{i \alpha}$.

(2) D3-instantons wrapping the exceptional divisors will have the minimal number of fermionic zero modes, and therefore contribute to the superpotential. To make this more precise, we need to consider the dual M-theory on a smooth Calabi-Yau fourfold, where the D3-instantons lift to M5-instantons. In this context it has been shown that if the M5 wraps a divisor satisfying $h^{1,0}=h^{2,0}=h^{3,0}=0$ (which in particular implies that its holomorphic Euler characteristic $\chi_{h} \equiv \sum_{i}(-1)^{i} h^{0, i}$ equals 1$)$, there is a contribution to

4 By asymmetric resolution in the compact model, we mean the resolution obtained by blowing up each local patch in the same asymmetric way. In principle there could be mixed symmetric/asymmetric resolutions, but we will not consider these. 
the superpotential [101]. In section 3, we will prove in detail that this is indeed the case for the lifts of the D3-instantons wrapped on the exceptional divisors. We also give a short argument below.

There are in fact other consistency conditions that need to be fulfilled. We will discuss these in section 6 .

\section{3. $M / F$-theory description of the model}

Type IIB string theory on the $Y / \mathbb{Z}_{2}$ orientifold is dual to M-theory on an elliptically fibered Calabi-Yau fourfold with base $B=Y / \mathbb{Z}_{2}$, in the limit of vanishing fiber area. The dual fourfold is easily constructed in this case [90]: it is simply $Z=\left(Y \times T^{2}\right) / \mathbb{Z}_{2}$, where the $\mathbb{Z}_{2}$ acts as our orientifold involution $\Omega$ on $Y$, and as $z \rightarrow-z$ on $T^{2}$. This gives a singular fourfold, with elliptic fibers degenerating to a $D_{4}$ singularity on top of the divisors $D_{i \alpha}$, and, in the symmetric resolution, a degenerate fiber with four terminal $\mathbb{Z}_{2}$ singularities on top of each fixed point in $B$. It can be considered as a partial resolution of $T^{8} / \mathbb{Z}_{2}^{3}$. Again this is an example of a Borcea-Voisin model [21, 100].

To rigorously address the question whether the lifts of the D3-instantons have the required properties mentioned in the previous subsection, one needs to resolve this fourfold in a way that preserves the elliptic fibration. This is somewhat tricky, and will be the subject of section 3. The basic idea is simple however. On the double cover $T^{2} \times Y$ of $Z$, the M5-brane lift of a D3 instanton wrapped on a divisor $E$ is $\widetilde{W}=T^{2} \times E$. As argued in section $2.2, h^{i, 0}(E)=0$ for $i>0$ and $E$ any of the divisors of interest discussed there. So the only harmonic $(i, 0)$-form on $\widetilde{W}$ is the $(1,0)$-form $d z$ living on $T^{2}$. Considering now the quotient $W=\widetilde{W} / \mathbb{Z}_{2}$ in $Z$, we see that $d z$ is odd and thus gets projected out. Moreover, blowing up the quotient singularities of $W$ will only change $h^{1,1}$. Hence, also after resolving the fourfold, $h^{i, 0}(W)=0$.

Another important point in the arguments we will give for the nonvanishing of the instanton conributions is the fact that the M5-branes under considerations have trivial third cohomology. This can be argued similarly. On $\widetilde{W}=T^{2} \times E$, the third cohomology is given by the product of $H^{1}\left(T^{2}\right)$ and $H^{2}(E)$. But quotienting by $\mathbb{Z}_{2}$ projects out every such class because the elements of $H^{2}(E)$ are even and those of $H^{1}\left(T^{2}\right)$ are odd. Furthermore, blowing up will not add any new 3-cycles. So $H^{3}(W)$ is trivial also after resolving the fourfold. A more precise discussion will be given in section 3 . 


\subsection{D3 tadpole and O3-curvature transition}

We now compute the D3 tadpole $Q_{3}$ measured in the quotient $Y / \mathbb{Z}_{2}$ (as usual, in the double cover $Y, Q_{3}$ is twice this). In the symmetric resolution, we have O3-planes. Choosing these to be non-exotic, their contribution to the D3-brane charge is

$$
Q_{3}\left(\mathrm{O}^{-}\right)=-\frac{1}{4} \times 64=-16
$$

The 7-branes also contribute to the D3 tadpole, through the "anomalous" couplings of RR-fields to worldvolume curvature [55,28, 95, 29,23, 24]. In a Calabi-Yau threefold, a single D-brane wrapped around a divisor $D$ thus contributes a D3-charge $-\chi(D) / 24$, and an O7plane $-4 \chi(D) / 24$. The total contribution from an $\mathrm{O} 7+S O(8) \mathrm{D} 7$ stack wrapped on $D$ is therefore $-12 \chi(D) / 24$ in $Y$, and half of that in the quotient. Hence the total 7-brane contribution is

$$
Q_{3}(7)=-\frac{1}{4} \sum_{i, \alpha} \chi\left(D_{i \alpha}\right) .
$$

In the symmetric resolution, $D_{i \alpha}$ has topology $\mathbb{P}^{1} \times \mathbb{P}^{1}$, so $\chi\left(D_{i \alpha}\right)=4$ and

$$
Q_{3}(7, \mathrm{symm})=-12 .
$$

There can also be contributions from the (half-integral quantized) $B$-field to various tadpoles, as well as from gauge instantons on the D7-branes, but we will take $\left.(B-F)\right|_{D_{i}}=0$ here, in which case there are no tadpole contributions of this kind. Combining O3 and 7 -brane contributions in this case gives

$$
Q_{3}(\text { tot })=-28
$$

In the asymmetric resolution $\chi\left(D_{1}\right)=\chi\left(D_{3}\right)=4$ and $\chi\left(D_{2}\right)=4+16=20$, so

$$
Q_{3}(\text { tot })=Q_{3}(7, \text { asymm })=-4-4-20=-28 \text {. }
$$

This agreement of tadpoles in symmetric and asymmetric resolutions can be understood locally: when flopping one local patch from fig. $3(a)$ to $(b)$, one O3 disappears from the corresponding orientifold, so $Q_{3}$ increases by $1 / 4$ in (2.12), but at the same time the Euler characteristic of the 7-branes wrapped around $D_{2}$ changes: a point gets blown up, which increases $h^{1,1}$ and therefore $\chi$ by 1 , so $Q_{3}$ decreases by $1 / 4$ in (2.13), and the total charge $Q_{3}$ is conserved. If this transformation can be realized physically, this is a rather 
interesting phenomenon, in which a 7-brane stack "eats" an O3 and blows up to conserve the net D3-charge.

This value of the tadpole fits nicely with the fourfold description. In this picture, the D3 tadpole is given by

$$
Q_{3}=-\frac{\chi(Z)}{24}
$$

The Euler characteristic $\chi(Z)$ of the fourfold say for the asymmetric resolution can be computed for example as follows. Removing the divisors $D_{i \alpha}$ from the base together with the $D_{4}$ fibers on top gives a space which is a direct product with a $T^{2}$ factor. This has Euler characteristic 0 . The Euler characteristic of the full space is therefore the sum of the Euler characteristics of the $D_{i \alpha}$ times the Euler characteristic of the $D_{4}$ fiber, which is 6 (it can be thought of as a collection of 5 spheres connected along 4 double points according to the extended $D_{4}$ Dynkin diagram). So $\chi(Z)=672$ and $Q_{3}=-28$. A similar match can be made for the symmetric resolution after properly taking into account the contribution to $\chi(Z)$ from terminal $\mathbb{Z}_{2}$ singularities [32].

The D3-tadpole thus produced can be canceled by adding 28 independent mobile D3branes, or by turning on RR and NSNS 3-form fluxes. This is further discussed in section 5.

In the symmetric resolution, we could also have chosen our O3-planes to be exotic. This does not change the geometry; it merely corresponds to turning on (torsion) twisted cohomology classes for the field strengths $H_{3}$ and/or $F_{3}$ in $H^{3}\left(\mathbb{R P}^{5}, \widetilde{\mathbb{Z}}\right)$, where $\mathbb{R P}^{5}=$ $S^{5} / \mathbb{Z}_{2}$ surrounds the $\mathrm{O} 3$ in $Y / \mathbb{Z}_{2}$ [104, 60, 11]. In the M-theory dual this corresponds to turning on torsion $G$-flux around the terminal $\mathbb{Z}_{2}$-singularities 93 .

The D3-charge of any exotic O3 has the opposite sign of a normal O3. The total tadpole in this case is thus

$$
Q_{3}=+16-12=+4 \text {. }
$$

To cancel this, one needs 4 anti-D3 branes, which breaks supersymmetry. Incidentally, the O3s are required to be exotic for a consistent CFT description at the orbifold point, as we will discuss further in section 4 . But, as stressed at the beginning of this section, it should not surprise us to find different consistent models at large radius. 


\section{Fourfold Geometry}

In this section we describe the geometry of the resolved Calabi-Yau fourfold. We describe the symmetric and asymmetric resolutions of the $T^{8} / \mathbb{Z}_{2}^{3}$ orbifold, and the birational transformation relating the two resolutions.

To simplify the presentation, we start with a lower dimensional orbifold, $T^{6} / \mathbb{Z}_{2} \times \mathbb{Z}_{2}$, which is dual to IIB on an orientifold of $T^{4} / \mathbb{Z}_{2}$. We discuss the resolution of the orbifold and the properties of the exceptional divisors introduced in the blow-up process.

We then move to our main example, the $T^{8} / \mathbb{Z}_{2}^{3}$ orbifold. We present two distinct resolutions and discuss their elliptic fibration structure. Starting from local models, we discuss the birational factorizations of the transformations relating the elliptically fibered Calabi-Yau fourfolds and their bases. This somewhat technical analysis is necessary to prove that the exceptional divisors $\mathcal{E}_{\bullet}$. in the (singular) symmetric resolution have the right topological properties to contribute to the nonperturbative superpotential: they have holomorphic Euler characteristic 1 and the higher cohomology groups $H^{0, i}\left(\mathcal{E}_{\bullet \bullet}\right), i=1,2,3$ vanish. We also show that their third cohomology is trivial, which is important for arguing that the instanton prefactor is nonvanishing.

\subsection{Lower dimensional orientifold}

It is instructive to consider first the lower dimensional analog, namely F-theory on $T^{6} / \mathbb{Z}_{2} \times \mathbb{Z}_{2}$. The action of the orbifold group $\Gamma=\mathbb{Z}_{2} \times \mathbb{Z}_{2}$ is presented in (2.1). We can view $T^{6} / \mathbb{Z}_{2} \times \mathbb{Z}_{2}$ as an elliptic fibration over $T^{4} / \mathbb{Z}_{2} \times \mathbb{Z}_{2}$ : let $z_{1}$ and $z_{2}$ be the coordinates on the base and $z_{3}$ be the coordinate on the elliptic fiber. Then, the elliptic fiber degenerates to type $I_{0}^{*}$ fibers $[$ [72] along the fixed locus of $\alpha$ and $\beta$ in the base. In F-theory, such a singularity corresponds to an $S O(8)$ gauge group [26, 15].

The base is $\mathbb{P}^{1} \times \mathbb{P}^{1}$ and there are $2 \times 4$ lines of $I_{0}^{*}$ fibers intersecting at 16 points 6 . In order to obtain a smooth Calabi-Yau threefold, we need first to blow-up the base at these 16 points and then resolve the singularities of the elliptic fibration. Let us first discuss the blowing-up of the base $B=\mathbb{P}^{1} \times \mathbb{P}^{1}$.

5 That is, along the fixed point set, the fiber degenerates to $T^{2} / \mathbb{Z}_{2}$, which is a rational curve with four singular points.

6 The corresponding Weierstrass model describing the transverse collision of two $I_{0}^{*}$ fibers is not minimal. 


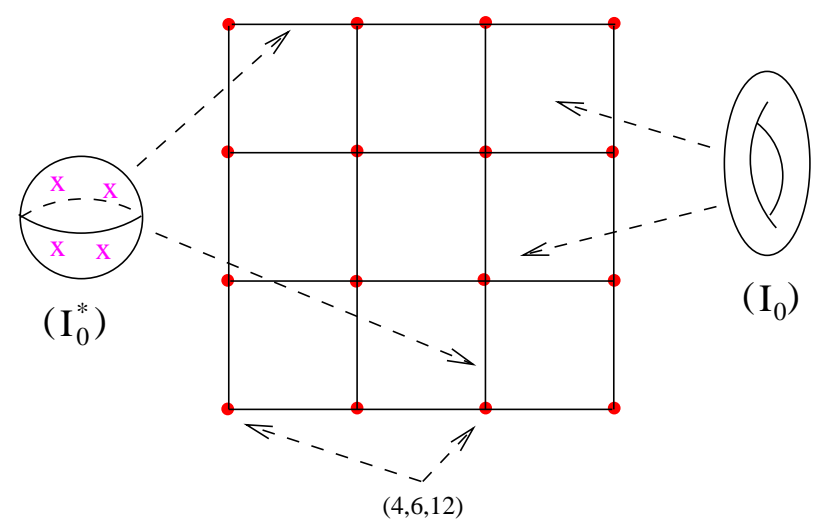

Fig. 4: Base of the elliptic threefold. There are 8 lines of $I_{0}^{*}$ singularities intersecting at 16 points, where the Kodaira vanishing orders are $(4,6,12)$.

We can work in local coordinates in the fibration $\left(\mathbb{C}^{2} \times T^{2}\right) / \mathbb{Z}_{2} \times \mathbb{Z}_{2}$ around the point $P=(0,0) \subset \mathbb{C}^{2}$, which lies at the intersection of the fixed lines $z_{1}=0$ and $z_{2}=0$. To describe the blow-up, introduce two coordinate patches $\left(t_{1}, z_{2}\right)$ and $\left(z_{1}, t_{2}\right)$ as follows:

$$
\left(z_{1}=t_{1} z_{2}, z_{2}=z_{2}\right) \text { and }\left(z_{1}=z_{1}, z_{2}=t_{2} z_{1}\right)
$$

The coordinates $t_{1}$ and $t_{2}=1 / t_{1}$ are homogeneous coordinates on the exceptional $\mathbb{P}^{1}$. The $\mathbb{Z}_{2}$ actions lift to the blown-up threefold; there are two fixed points on the exceptional $\mathbb{P}^{1}$ given by $t_{1}=0$ and $t_{2}=0$, where it intersects the unresolved singular divisors. The elliptic fiber over the exceptional $\mathbb{P}^{1}$ is smooth, except at the points $t_{1}=0$ and $t_{2}=0$, where there are $I_{0}^{*}$ singularities. We note that the elliptic fibration over the blown-up base admits a section. The blow-up process is ilustrated in the figure below.

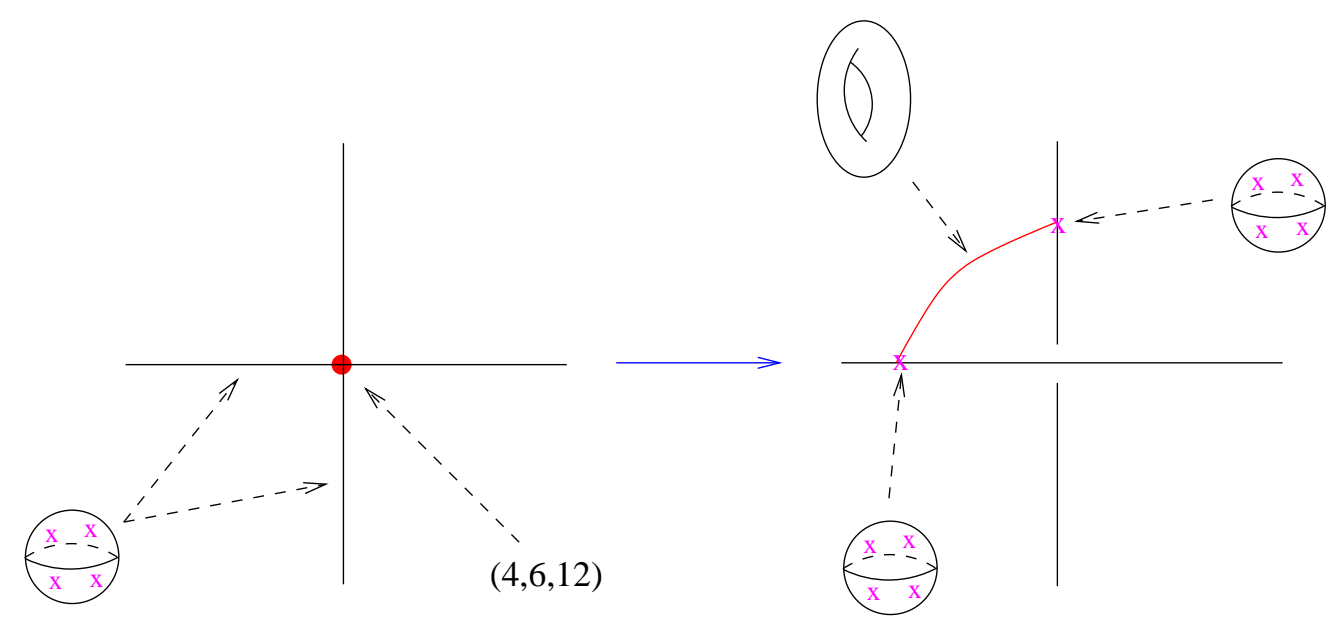

Fig. 5: Base blow-up at an $I_{0}^{*}-I_{0}^{*}$ collision point. 
Blowing-up the base introduce 16 new Kähler parameters. The next step is to resolve the elliptic fibration and this will introduce $8 \times 4=32$ additional Kähler parameters since after the blow-ups in the base there are 8 isolated curves on top of which the elliptic fiber is type $I_{0}^{*}$. Taking into account the original 2 Kähler parameters of the base and the section, we recover the 51 Kähler parameters of the resolution. We have obtained a smooth threefold that is elliptically fibered and is one of the Borcea-Voisin models [21,100].

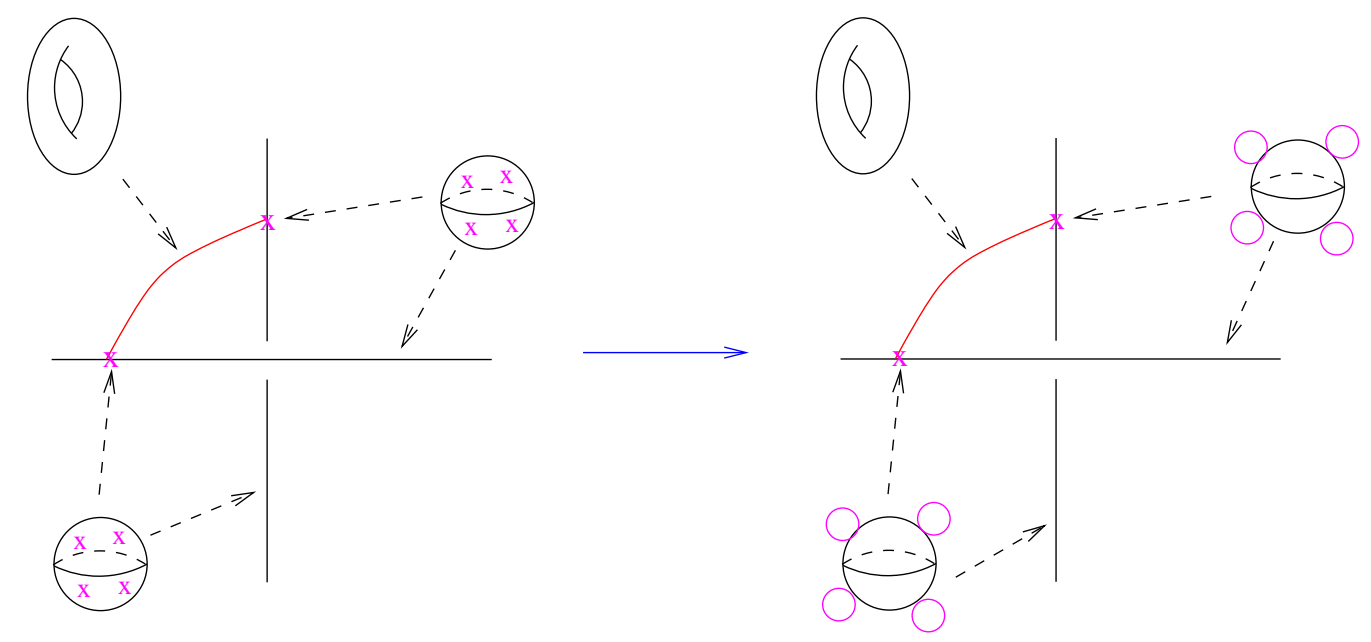

Fig. 6: Fiber blow-ups.

The process of resolving the elliptic fiber will turn the elliptic fibration over the exceptional divisors in the base into rational elliptic surfaces $S_{i}, i=1, \ldots, 16$, that is del Pezzo surfaces $d P_{9}$. These have $h^{1,0}(S)=h^{2,0}(S)=0$.

In type IIB theory language, the description of the orientifold of $T^{4} / \mathbb{Z}_{2}$ is as follows [52]. The fixed curves are wrapped by D7-branes sitting on top of orientifold O7 planes, and on the worldvolume of each 7-brane there is an $S O(8)$ gauge theory. After blowing-up the base, the gauge theory is $S O(8)^{8}$ with no matter.

\section{2. $T^{6} / \mathbb{Z}_{2} \times \mathbb{Z}_{2}$ orientifold}

Proceeding analogously to the previous section, consider now the orbifold $Z=$ $T^{8} /\left(\mathbb{Z}_{2}\right)^{3}$. The orbifold group acts as

$$
\begin{array}{ccccc} 
& z_{1} & z_{2} & z_{3} & z_{4} \\
\alpha & + & - & - & + \\
\beta & - & + & - & + \\
\Omega & - & - & - & -
\end{array}
$$


This is another example of the Borcea-Voisin construction [21,100]. To get our Calabi-Yau fourfold start with the Calabi-Yau orbifold $Y=T^{6} / \mathbb{Z}_{2} \times \mathbb{Z}_{2}$ with the orbifold action given by (3.1) and construct $Z=Y \times T^{2} /(\sigma,-\mathbb{1})$, where $\sigma$ is an involution of $Y$ that changes the sign of the holomorphic three-form.

The local singularities are of the form $\mathbb{C}^{4} /\left(\mathbb{Z}_{2}\right)^{3}$. The figure below presents the toric resolutions of the singularities. We see again that the local singularities do admit crepant resolutions and it is possible to glue them together and get a smooth crepant resolution $\widetilde{Z} \rightarrow Z$ with $h^{1,1}(\widetilde{Z})=100, h^{2,1}(\widetilde{Z})=0, h^{3,1}(\widetilde{Z})=4, h^{2,2}(\widetilde{Z})=460$ and $\chi(\widetilde{Z})=672$.
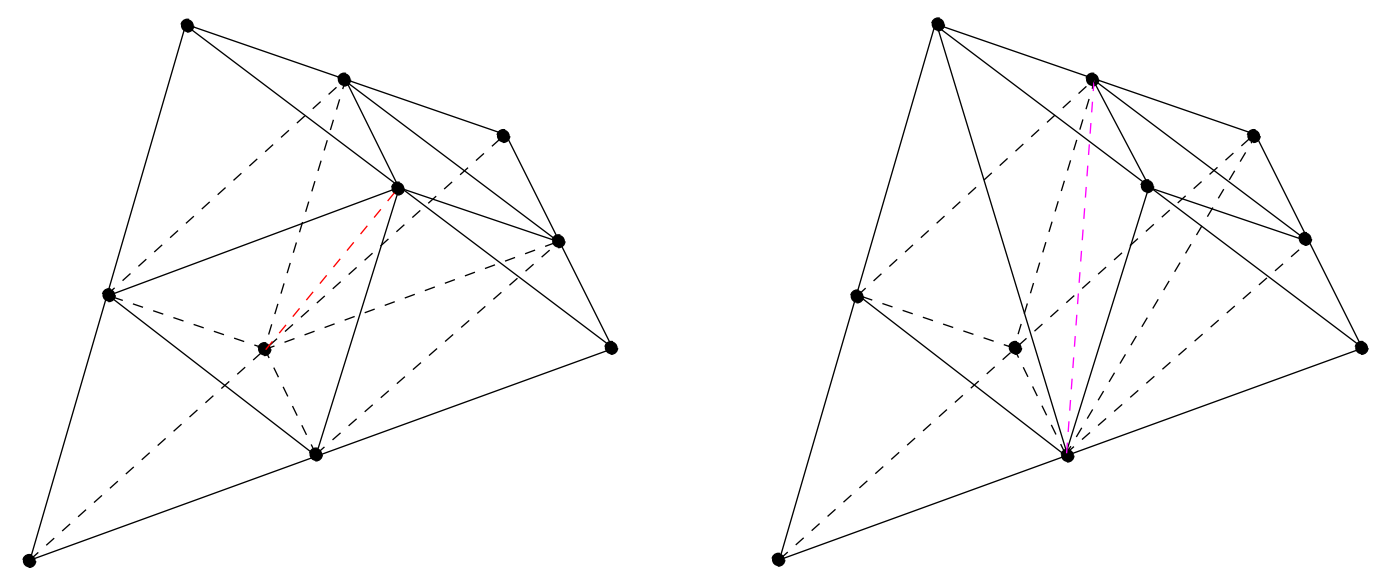

Fig. 7: Symmetric and asymmetric resolutions of $\mathbb{C}^{4} /\left(\mathbb{Z}_{2}\right)^{3}$.

We can also think of $Z=T^{8} /\left(\mathbb{Z}_{2}\right)^{3}$ as an elliptic fibration over $T^{6} /\left(\mathbb{Z}_{2}\right)^{3}$, with $I_{0}^{*}$ fibers along the fixed point set of $\alpha \circ \Omega, \beta \circ \Omega$ and $\alpha \circ \beta \circ \Omega$.

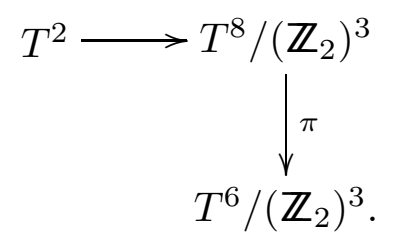

The base is $\mathbb{P}^{1} \times \mathbb{P}^{1} \times \mathbb{P}^{1}$ and the structure of the fixed point set is presented in fig. 8 below. There are 12 planes of $I_{0}^{*}$ singularities intersecting along 48 lines where the Kodaira vanishing orders are $(4,6,12)$ and the Weierstrass model is not minimal. There are 64 points where three such lines meet. This is in fact an orientifold of $T^{6} / \mathbb{Z}_{2} \times \mathbb{Z}_{2}$. 


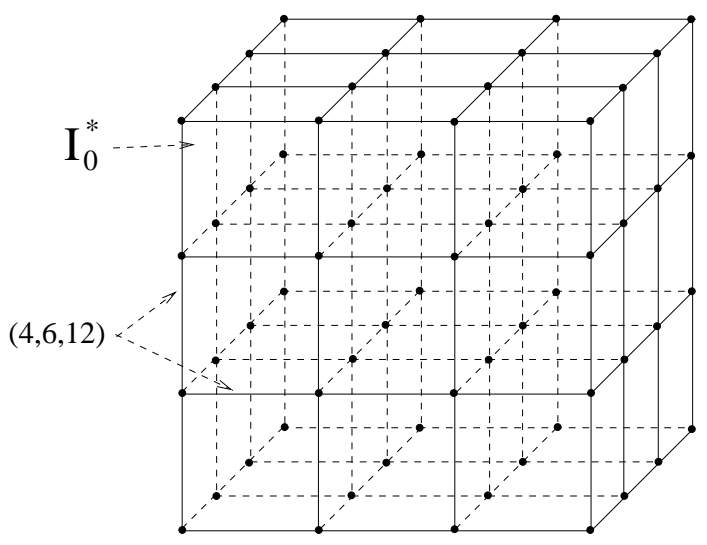

Fig. 8: Base of the elliptic fourfold. There are 12 planes of $I_{0}^{*}$ singularities intersecting along 48 lines where the Kodaira vanishing orders are $(4,6,12)$. There are 64 points where three such lines meet.

We would like to resolve the singularities of the fourfold while preserving the elliptic fibration structure, such that we can consider a compactification of F-theory on $\widetilde{Z}$. We first analyze the local geometry of three intersecting planes of $I_{0}^{*}$ singularities. In order to understand the behavior of the elliptic fibration under base blow-ups, we consider the local geometry of three intersecting planes of $I_{0}^{*}$ singularities modeled by the following Weierstrass equation

$$
y^{2}=x^{3}+x s_{1}^{2} s_{2}^{2} s_{3}^{2}+s_{1}^{3} s_{2}^{3} s_{3}^{3},
$$

where $s_{1}, s_{2}, s_{3}$ are affine coordinates along the three coordinate lines. The situation is presented in fig. 9 below.

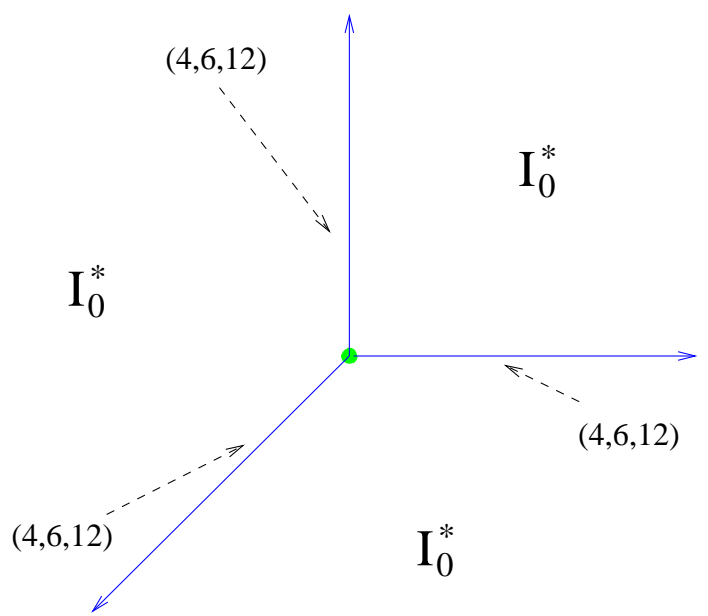

Fig. 9: Local geometry of three intersecting planes of $I_{0}^{*}$ singularities. Along the coordinate axes, the Kodaira vanishing orders are $(4,6,12)$. 


\subsection{The base threefolds}

In order to obtain a smooth Calabi-Yau fourfold, we need to blow-up the 48 lines in the base on top of which the Weierstrass model is not minimal. In the local model, the base is $\mathbb{C}^{3}$ blown-up along the three coordinate lines. These blow-ups are toric and the different triangulations correspond to different topologies of the noncompact base and are obtained by performing different sequences of birational transformations. The two different types of triangulations are presented in the figure below. Note that the asymmetric triangulation corresponds to a smooth geometry of the base, which we denote $B_{+}$, while the symmetric one correspond to a singular one, and we denote this base by $B$. To see this, note that there is a $\mathbb{Z}_{2}$ singularity in the coordinate patch associated with the cone $E_{12} E_{23} E_{31}$.

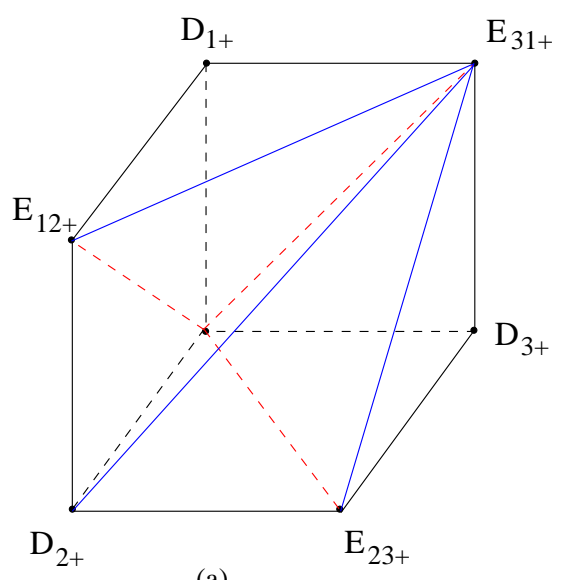

(a)

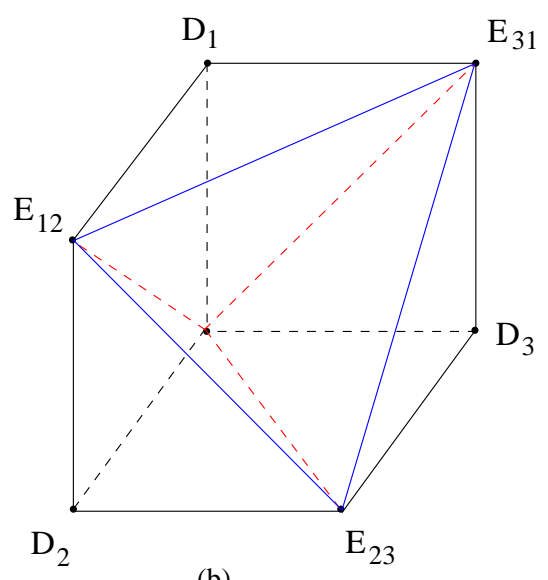

(b)

Fig. 10: Toric description of $\mathbb{C}^{3}$ blown-up long the 3 coordinate axes. (a) Asymmetric and (b) symmetric triangulations.

It is again useful to draw the dual diagrams illustrating the compact and noncompact cycles of the local base geometries. These are presented in the figure below, which is essentially a $\mathbb{Z}_{2}$ quotient of fig. 3 . The $\mathbb{Z}_{2}$ is the orientifold action and $Y_{+} \longrightarrow B_{+}$and $Y \longrightarrow B$ are $2: 1$ branched coverings.

The birational transformation between $B_{+}$and $B$ is no longer a flop. To see this, note that the curve $\Gamma_{+}$has normal bundle $N_{\Gamma_{+} / B_{+}}=\mathcal{O}_{\Gamma_{+}}(-1) \oplus \mathcal{O}_{\Gamma_{+}}(-2)$ and

$$
K_{B_{+}} \cdot \Gamma_{+}=1
$$

On the other hand,

$$
K_{B} \cdot \Gamma=-\frac{1}{2} .
$$




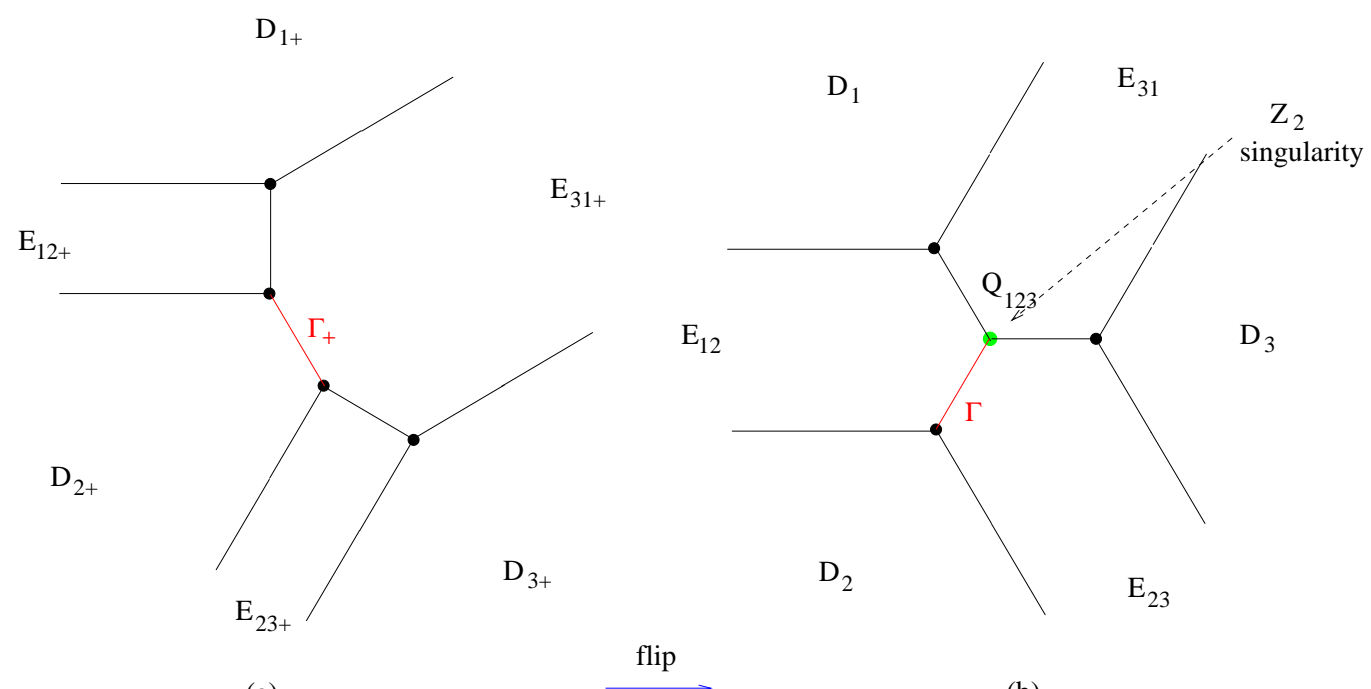

(a)

(b)

Fig. 11: (a) Asymmetric and (b) symmetric blow-ups: divisors and curves.

This is a flip transformation; there exist birational morphisms $B \rightarrow \bar{B}$ and $B_{+} \rightarrow \bar{B}$ contracting $\Gamma$ and $\Gamma_{+}$respectively .

We now recall some basic facts about the flip as a $\mathbb{Z}_{2}$ quotient of the flop. We follow the construction in [73] and we first describe the flop. In the following we will restrict to neighborhoods of the curves of interest. We will abuse notation and denote these neighborhoods by the same symbols used for the corresponding varieties discussed until now, which contain these neighborhoods as subsets. Consider $\bar{Y}$ defined by the equation $(x y-u v=0) \subset \mathbb{C}^{4}$. The origin is an isolated singularity. We can resolve the singularity by blowing up the origin in $\mathbb{C}^{4}$ : the exceptional locus in the resolved threefold $\widetilde{Y}$ is isomorphic to a projective quadric in $\mathbb{P}^{3}$, defined by the projective equation $x y-u v=0$.

By blowing up the the plane $x=v=0$ we obtain a small resolution $Y$ of $\bar{Y}$; by blowing up the plane $x=u=0$ we have another small resolution $Y_{+}$isomorphic to $Y$ outside the locus of the exceptional curves. The birational transformation

$$
Y \leftarrow \cdots \rightarrow Y_{+}
$$

is a flop. The equation of $Y_{+}$and $Y$ are respectively:

$$
\begin{aligned}
& \lambda v-\mu x=0, \quad \lambda y-\mu u=0 \\
& \lambda u-\mu x=0, \quad \lambda y-\mu v=0 .
\end{aligned}
$$


Consider now the $\mathbb{Z}_{2}$ action on $\mathbb{C}^{4}$ defined by $(x, y, u, v) \rightarrow(-x, y,-u, v)$. This involution induces an action on $\bar{Y}, Y, Y_{+}$and $\widetilde{Y}$. The fixed loci of the actions are respectively: - On $\bar{Y}$ : The plane $x=u=0$.

- On $\tilde{Y}$ : The strict transform of the plane $x=u=0$ and on the exceptional projective quadric the lines $x=u=0$ (at the intersection with the fixed plane) and $y=v=0$.

- On $Y$ : The strict transform $D$ of the plane $x=u=0$, and a point $Q$ on the exceptional $\mathbb{P}^{1}$ of the small resolution $Y \rightarrow \bar{Y}$. This point is the the image of the fixed line $y=v=0$ on the exceptional quadric.

- On $Y_{+}$: The strict transform $D_{+}$of the plane $x=u=0 ; D_{+}$is a plane blown up at a point, which contains the exceptional rational curve. The other fixed line on the exceptional quadric has been mapped surjectively onto the line at the intersection of the quadric and the fixed plane: the image curve is the exceptional $\mathbb{P}^{1}$ of the small resolution $Y_{+} \rightarrow \bar{Y}$.

Let $\bar{B}, B, B_{+}$and $\widetilde{B}$ the quotient threefolds by these actions:

- $B$ is singular at the fixed point $Q$ (which is contained on the flipped curve); $Q$ is the image of the fixed line $y=v=0$ on the exceptional quadric.

- $B_{+}$is smooth.

This analysis can be trivially extended to the noncompact geometries that are of interest to us and is in agreement with the toric description in fig. 11. We now proceed with the study of the elliptically fibered fourfolds over $B_{+}$and $B$.

\subsection{The elliptic fourfolds}

We start with the elliptic fibration over $B_{+}$

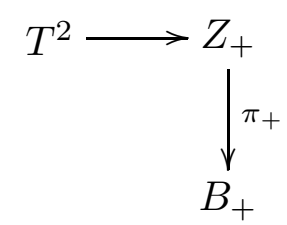

and perform successively the blow-ups along the three coordinate lines according to the sequence presented below. The figure also indicates how the Kodaira type of the elliptic fiber on top of the divisors in the base changes at every step of the the blow-up sequence. We find that the blow-up geometry contains three exceptional divisors on top of which the elliptic fiber is smooth. The proper transforms of the surfaces of $I_{0}^{*}$ singularities do not intersect any longer. 

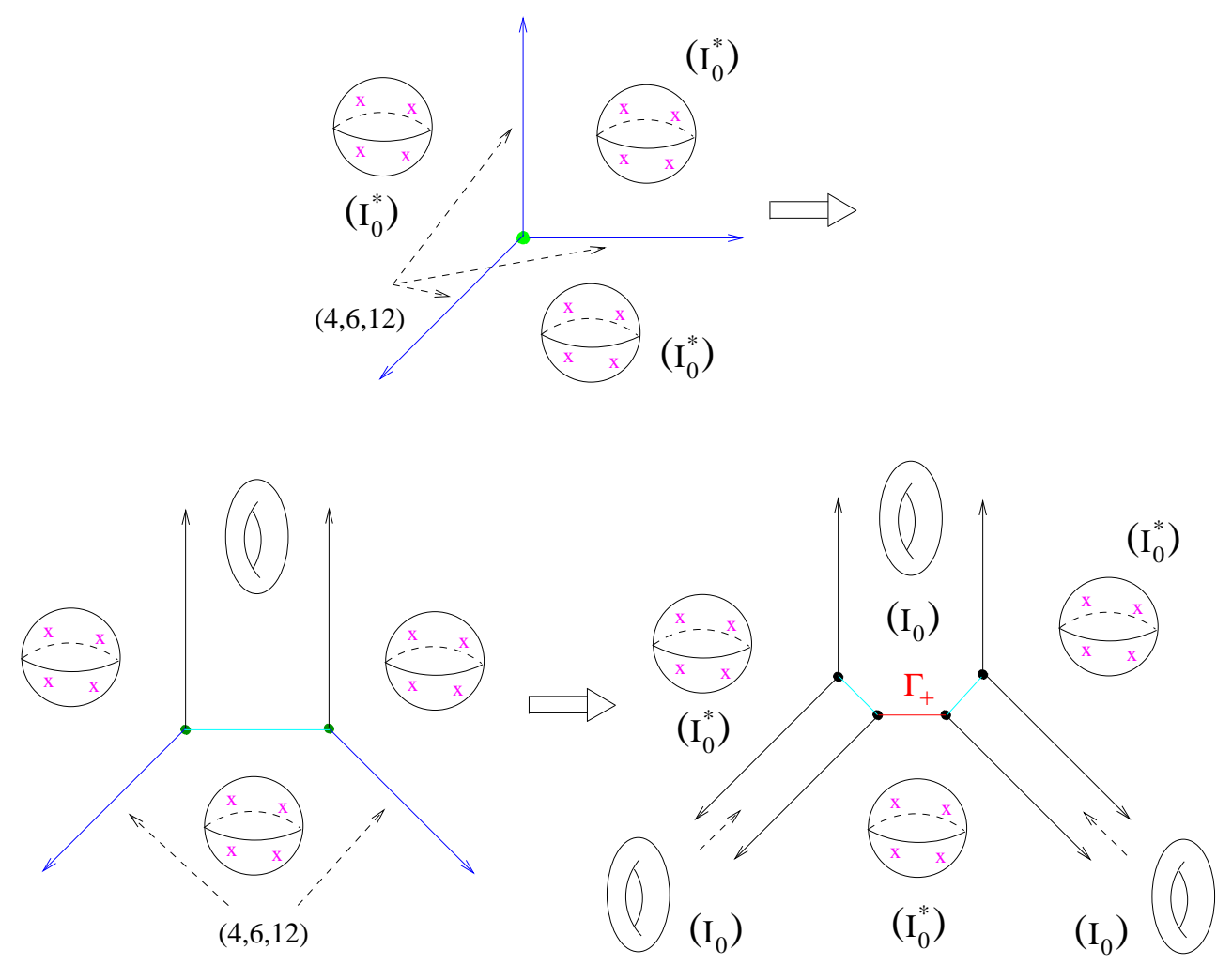

Fig. 12: Local sequence of blow-ups in the base, asymmetric phase. The fiber is smooth on top of the exceptional divisors and the surfaces of $I_{0}^{*}$ singularities do not intersect any longer.

This local picture is suggestive of the strategy we need to follow when blowing- up the base of the compact fourfold. That is, we first perform the blow-ups along the 16 curves in a given homology class and then blow-up the remaining two sets of 16 curves. Then, there will be 16 exceptional divisors that are del Pezzo surfaces $d P_{9}$ and 32 exceptional divisors that are Hirzebruch surfaces $\mathbb{F}_{0}$. Moreover, the proper transforms of the $I_{0}^{*}$ surfaces are Hirzebruch surfaces $\mathbb{F}_{0}$ and $\mathbb{F}_{0}$ blown-up at 16 points.

The next step is to resolve the elliptic fibration. The analysis is similar to the one performed in the lower dimensional example. We find that the fourfold exceptional divisors $\mathcal{E}_{\bullet \bullet+}$ are either threefolds isomorphic to $\mathbb{P}^{1} \times d P_{9}$ or blow-ups of $\mathbb{P}^{1} \times d P_{9}$ along 8 (reducible) rational curves. They are therefore rational and have holomorphic Euler characteristic 1. In fact, in the case of the smooth elliptic fourfold we can arrive at this conclusion by performing a Riemann-Roch computation as in [54].

We would like to understand the elliptic fibration over the singular base $B$ along the same lines. It turns out that it is a bit subtle to understand how the fibration behaves under the sequence of birational transformations that lead to $B$. In order to construct an 
elliptic fourfold, let us return to the local picture used in the previous subsection. Consider the trivial elliptic fourfolds $\bar{Y} \times T^{2}, Y \times T^{2}, Y_{+} \times T^{2}$ and $\widetilde{Y} \times T^{2}$; let us consider a $\mathbb{Z}_{2}$ action, defined on the first factor as the action defined in the previous subsection and on the second factor as the standard involution on the torus.

Now, let $\bar{W}, W, W_{+}$and $\widetilde{W}$ be the fourfolds obtained by quotienting this action: they are naturally elliptically fibered over $\bar{B}, B, B_{+}$and $\widetilde{B}$. The singular fibers map over the fixed locus of the quotient action as follows.

In $Y_{+} \times T^{2} \rightarrow Y_{+}$the fixed locus is $D_{+} \subset Y_{+}$; in the quotient $W_{+} \rightarrow B_{+}$the fibers are double rational curves with 4 singular points, over the points in $D_{+}$. The minimal resolution of $W_{+}$is a smooth fourfold $Z_{+}$, the singular fibers over $D_{2}$ in $Z_{+}$are of Kodaira type $I_{0}^{*}$.

In $Y \times T^{2} \rightarrow Y$ the fixed locus is the strict transform $D$ of the plane $x=u=0$ and the fixed point $P$ : in the quotient $W \rightarrow B$ the fibers are double rational curves with 4 singular points over each point in $D$ and the point $Q$. The (minimal) smooth resolution of $W$ is a singular fourfold $Z: Z$ is smooth over $D$, the singular fibers are of Kodaira type $I_{0}^{*}$, while the singular isolated fiber over $Q$ has 4 singular points, these 4 singularities are terminal (already in $W$ ) [85]. These are the only singularities of $Z$.

Again, this local analysis extends trivially for the noncompact geometries we are interested in. In the smooth case, it agrees with the previous description of the elliptic fibration. In the singular case, we learn that the elliptic fibration is as in the figure below.

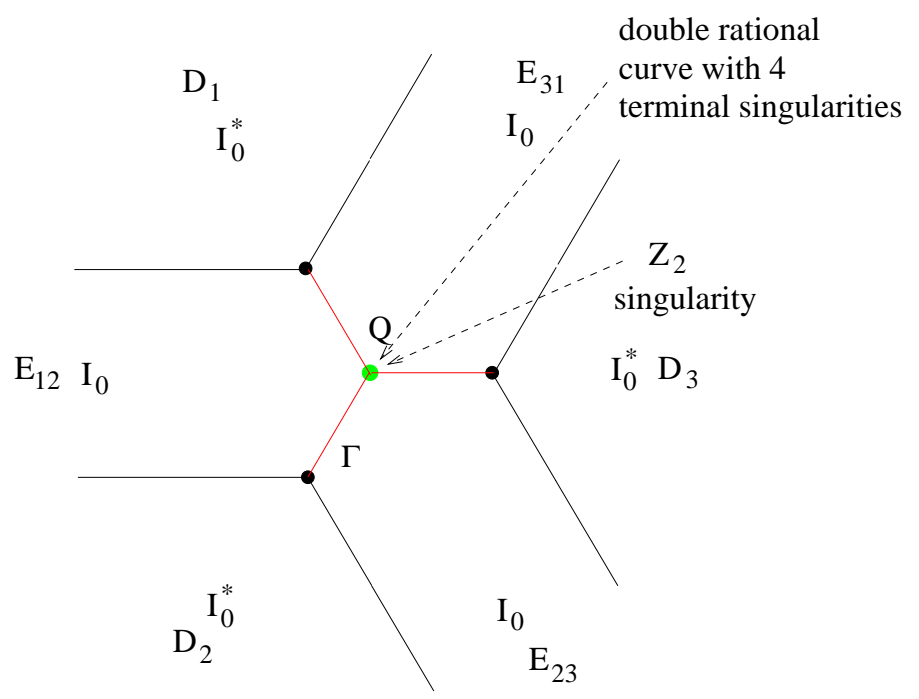

Fig. 13: Elliptic fibration over the singular base. The fiber is generically smooth on top of the exceptional divisors, but degenerates to a double rational curve with 4 terminal singularities over the $\mathbb{Z}_{2}$ singularity in the base $B$. The surfaces of $I_{0}^{*}$ singularities do not intersect any longer. 
In the following sections we describe in detail a birational factorization of the flop between the elliptic fourfolds $\pi: Z \rightarrow B$ and $\pi_{+}: Z_{+} \rightarrow B_{+}$as a sequence of blow-ups and contractions. As in the previous sections, it is sufficient to consider the local situation; we start by describing the factorization of the threefold flip.

The objects of particular interest are the threefold divisors in $Z$ and $Z_{+}$of holomorphic Euler characteristic 1: we saw that $\mathcal{E}_{31+}=\pi_{+}^{-1}\left(E_{31+}\right) \subset Z_{+}$is a smooth threefold (in the smooth fourfold $Z_{+}$) with holomorphic Euler characteristic 1. From the explicit description of the fourfold flop it will follow that the strict trasform of $\mathcal{E}_{31+} \subset Z_{+}$is $\mathcal{E}_{31}=\pi^{-1}\left(E_{31}\right) \subset Z$ and that $\mathcal{E}_{31}$ is also a divisor (in the singular fourfold $Z$ ) with holomorphic Euler characteristic 1.

\subsection{A birational factorization of the threefold flip}

We consider the asymmetric resolution $B_{+}$and perform two blow-ups followed by two birational contractions. The composition of these morphisms gives a birational factorization of the flip. The geometry can be easily seen from the pictures below. The existence of the blow-ups is clear: to see the contractions we can either consider a toric picture, or invoke the minimal model contraction algorithm with its log variation. We describe the contraction algorithm because it can also be used to study the transformation between our fourfolds.
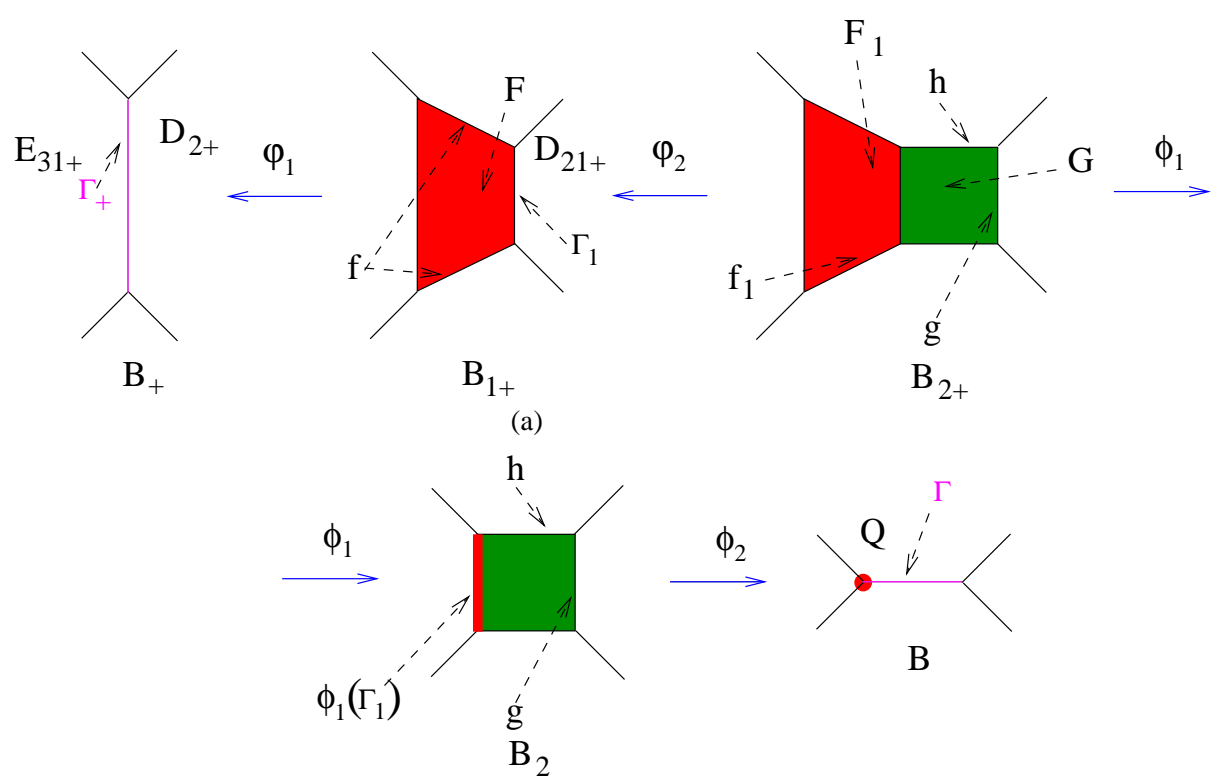

(b)

Fig. 14: $B_{+}$blown-up and contracted to $B$. (a) Threefolds blow ups. (b) Threefold contractions. 
- (The first blow up.) In $B_{+}$we first blow up $\Gamma_{+}$and denote by $\varphi_{1}: B_{1+} \rightarrow B_{+}$the corresponding contraction morphism; since $\Gamma_{+}$is a smooth rational curve with normal bundle $(-2,-1)$, the exceptional divisor $F$ is isomorphic to the Hirzebruch surface $\mathbb{F}_{1}$; let $f$ denote the fiber of the ruling. Let $D_{21+}$ be the strict transform of $D_{2+}$ in $B_{1+}$. Let $\Gamma_{1}$ be the unique irreducible curve with negative self intersection on $F$, then: $\left(\Gamma_{1}\right)^{2}{ }_{\mid F}=-1$ and $\Gamma_{1}=F \cap D_{21+}$. Note that $\Gamma_{1}$ is a smooth rational curve with normal bundle $(-1,-1)$. - (The second blow up.) In $B_{1+}$, let us blow up $\Gamma_{1}$ and denote by $\varphi_{2}: B_{2+} \rightarrow B_{1+}$ the corresponding contraction morphism; since $\Gamma_{1}$ is a smooth rational curve with normal bundle $(-1,-1)$, the exceptional divisor $G$ of $\varphi_{2}$ is isomorphic to the Hirzebruch surface $\mathbb{F}_{0}$. Let $F_{1}$ denote the strict transform of $F$ in $B_{2+}$, and $f_{1} \simeq f$ the strict transform of the ruling $f ; f_{1}$ is a smooth rational curve with normal bundle $(0,-2)$. In fact, if we denote by $H$ the strict transform of $E_{12+}$ in $B_{2+}, f_{1}=F_{1} \cap H$ and $\left(f_{1}\right)^{2}{ }_{\mid H}=-2$. By the adjunction formula $K_{B_{2+}} \cdot f_{1}=0$; note also that $H \cdot f_{1}=-2$.

- (The first contraction.) By the (log-)contraction theorem there exists a birational morphism $\phi_{1}: B_{2+} \rightarrow B_{2}$ contracting all the curves in the ruling $f_{1}$. The contraction $\phi_{1}$ is a log-terminal contraction; $B_{2}$ has $\mathbb{Q}$-factorial canonical singularities along $\phi_{1}\left(\Gamma_{1}\right)$, which is a smooth rational curve. ( $f_{1}$ is a curve with normal bundle $(0,-2)$ : which means it is a -2 curve in a generic surface $S$ that meets $F_{1}$ transversely along a fixed curve in the ruling $f_{1}$. Contracting the ruling $f_{1}$ will lead to an $A_{1}$ singularity in the image of the surfaces $S$ : in the threefold the singularity is $A_{1} \times \mathbb{P}^{1}$, where $\mathbb{P}^{1} \simeq \Gamma_{1}$.) This contraction is allowed since $f_{1}$ is a negative extremal ray in the Mori cone $\overline{N E}\left(B_{2+}\right)$. To see this, note that $f_{1}=\varphi_{2}^{*} f-h$, where $h$ is a ruling of $G$, and $\left(K_{B_{2+}}+1 / 2 F_{1}\right) \cdot f_{1}=-1$. For further reference, note also that $K_{B_{2+}}=\phi_{1}^{*}\left(K_{B_{2}}\right)$.

- (The second contraction.) Now we consider $\phi_{1}(G) \simeq \mathbb{F}_{0}$ and its ruling $g$ which is homologous to $\phi_{1}\left(\Gamma_{1}\right)$ in $\phi_{1}(G)$ (in fact $[g]=\left[\phi_{1}\left(\Gamma_{1}\right)\right]$ ). Again, by the contraction theorem there exists a birational morphism $\phi_{2}: B_{2} \rightarrow B$ contracting all the curves in the ruling $g$. In fact, $g$ is an extremal ray in the Mori cone $\overline{N E}\left(B_{2}\right)$ and $K_{B_{2}} \cdot g=-1$ (to see this, recall that $K_{B_{2+}}=\phi^{*}\left(K_{B_{2}}\right)$, the normal bundle of $g$ in $B_{2+}$ is $(0,-1)$ and use adjunction on the smooth threefold $\left.B_{2+}\right)$. In particular, $\phi_{2}\left(\phi_{1}\left(\Gamma_{1}\right)\right)=Q$ is a singular point: it can be verified that $B$ is smooth outside $Q$ (it follows from the Castelnuovo-Enriques contraction criterion, see [57]) and that the singularity at $Q$ is terminal.

To see that the singularity is terminal is enough to compare the pullback of the canonical divisor to the smooth resolution $\phi_{2} \cdot \phi_{1}: B_{2+} \rightarrow B$ with that of $B_{2+}: K_{B_{2+}}=$ $\left(\phi_{2} \cdot \phi_{1}\right)^{*}\left(K_{B}\right)+G$. For $h$, the other ruling of $G$, we have that $\phi_{2} \cdot \phi_{1}(h)=\Gamma$ is a smooth rational curve, and with the same methods it can also be verified that $K_{B} \cdot \Gamma=-1 / 2$, as claimed in the previous subsection. 


\subsection{The fourfold flop}

We first study the induced elliptic fibration $\pi_{2+}: Z_{2+} \rightarrow B_{2+} ; Z_{2+}$ is smooth and isomorphic to $Z_{+}$outside the exceptional locus. The fiber over a point in $G$ is a smooth elliptic curve, while the fiber over a point in $F_{1}$ is of type $I_{0}^{*}$. By construction, the fibers in $Z_{2+}$ over the points in $\Gamma_{+}, \Gamma_{1}$ and $f_{1}$ are all isomorphic to each other, and so are all the fibers over the points in $g$.

Note also that $K_{Z_{2+}}=\pi_{2+}^{*}\left(K_{B_{2+}}+\Delta_{2+}\right)$, where $\Delta_{2+}$ is supported on the ramification locus of the fibration, with suitable coefficients, determined by the Kodaira type fibers; for example, the coefficient of $F_{1}$ in $\Delta_{2+}$ is $1 / 2$. The birational morphism $Z_{2+} \rightarrow Z_{+}$is induced by the resolution of the pullback of $Z_{+}$. We want to show that there is a birational morphism $Z_{2+} \rightarrow Z$ induced by the contraction morphisms $\phi_{2} \cdot \phi_{1}$.

- (The first fourfold contraction, following $\phi_{1}$ ). The goal of these contractions if to construct a birationally equivalent elliptic fibration over $B_{2}$; the threefold over $F_{1}$ will be contracted to a singular surface over $\phi_{1}\left(\Gamma_{1}\right)$, in particular the surfaces in the fourfold over the fiber $f_{1}$ are contracted to a double rational curve with 4 marked points.

To see this, note first that $\pi_{2+}^{-1}\left(f_{1}\right)$ is a reducible surface, as illustrated in the picture below: the red vertical surface is isomorphic to $\mathbb{F}_{0}$ (this surface appears in the fourfold with multiplicity two and it is the strict transform of $\left.T^{2} / \mathbb{Z}_{2} \times \mathbb{P}^{1}\right)$; the four blue surfaces arise as resolution of the four fixed points in $T^{2}$, and each of these surfaces is isomorphic to $\mathbb{F}_{1}$. The negative sections of the $\mathbb{F}_{1}$ surfaces are exactly at the intersections of the blue surfaces with the (reduced) red surface.

Consider now a surface $S_{2+} \hookrightarrow B_{2+}$ intersecting $F_{1}$ and $G$ along their rulings and let $\mathcal{E}_{2 s}$ be the elliptic threefold defined by the following commutative diagram

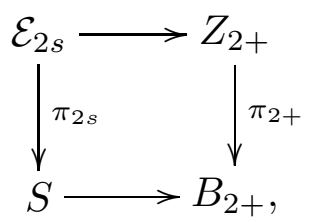

where the horizontal arrows are inclusions. We have $K_{\mathcal{E}_{2 s}}=\pi_{2 s}^{*}\left(K_{S}+\Delta_{S}\right)$ where $\Delta_{S}=$ $\Delta_{2+} \cap S$. Let $\gamma$ be a curve in ruling of $\mathbb{F}_{0}$ such that $\pi_{2 s}(\gamma)=f_{1}$; then by the projection formula we have $K_{\mathcal{E}_{2 s}} \cdot \gamma=\left(K_{S}+\Delta_{S}\right) \cdot \pi_{2 s *}(\gamma)=\Delta_{S} \cdot f_{1}=-1$ since the fibers over $f_{1}$ are type $I_{0}^{*}$ and $f_{1}$ does not intersect the proper transform of $D_{21+}$ under $\varphi_{2}$. By adjunction on $\mathcal{E}_{2 s}$ we then find that $N_{\gamma / \mathcal{E}_{2 s}}=\mathcal{O}_{\gamma}(0) \oplus \mathcal{O}_{\gamma}(-1)$, proving that the blue surfaces are isomorphic to $\mathbb{F}_{1}$. Now, in order to find the birational contractions we use 


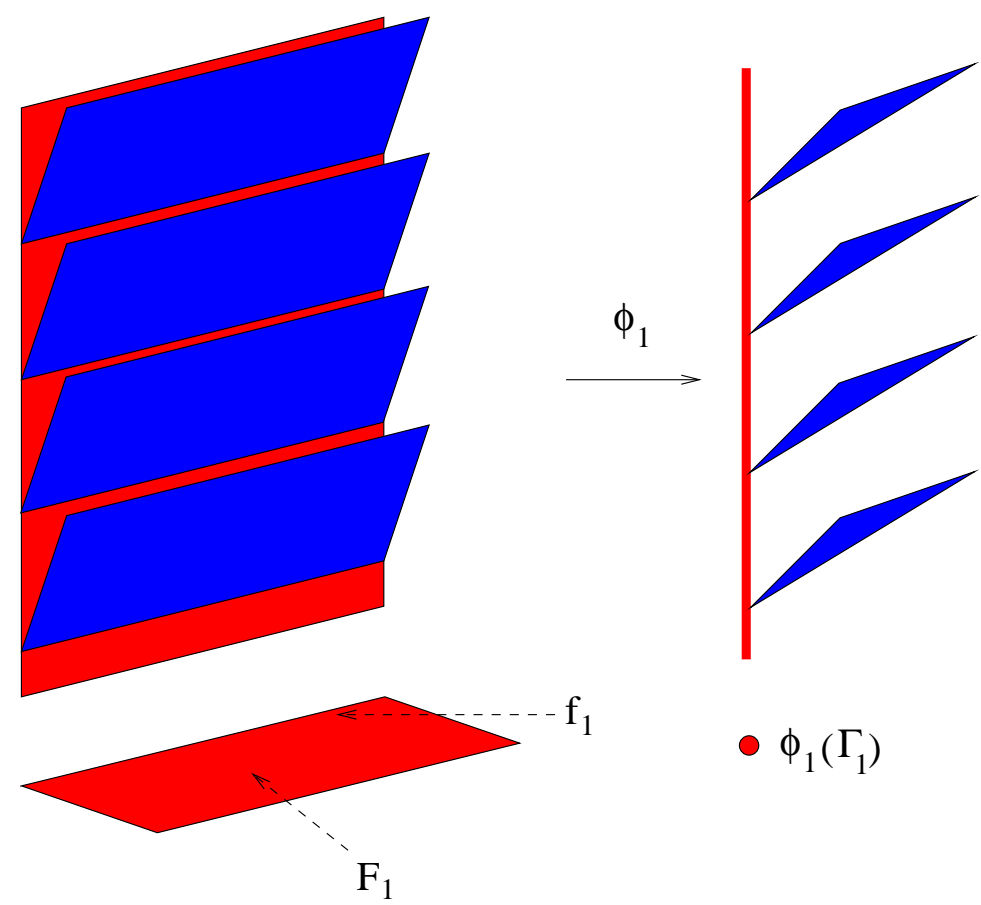

Fig. 15: The surface in $Z_{2+}$ over $f_{1} \in F_{1}$ and the first contraction, following $\phi_{1}$. The right hand side represents the fiber over $\phi_{1}\left(\Gamma_{1}\right)$.

again the minimal model contraction algorithm: by the projection formula: $K_{Z_{2+}} \cdot \gamma=$ $\pi_{2+}^{*}\left(K_{B_{2+}}+\Delta_{2+}\right) \cdot \gamma=\left(K_{B_{2+}}+\Delta_{2+}\right) \cdot f_{1}=\left(K_{B_{2+}}+1 / 2 F_{1}\right) \cdot f_{1}=-1$. Therefore, $\gamma$ is a negative extremal ray in the Mori cone $\overline{N E}\left(Z_{2+}\right)$, and by the contraction algorithm there exists a birational morphism which contracts all the curves in the homology class $\gamma$, to a smooth fourfold. The image of the red double threefold over $F_{1}$ is now a double surface isomorphic to $\mathbb{F}_{0}$. The blue surfaces become isomorphic to $\mathbb{P}^{2}$, represented by the blue triangles in fig. 15 .

The contraction algorithm ensures then the existence of another birational morphism contracting all these blue $\mathbb{P}^{2}$ 's, in the fourfolds (it can be checked that $N_{\mathbb{P}^{2} / \mathcal{E}_{2 s}}=$ $\mathcal{O}_{\mathbb{P}^{2}}(-2)$ ), to four smooth rational curves in $\mathbb{F}_{0}$ (in the same ruling). The resulting fourfold $Z_{2}$ is now singular along these four curves and the singularities are terminal; we also have an elliptic fibration $\pi_{2}: Z_{2} \rightarrow B_{2}$. The fiber over $\phi_{1}\left(\Gamma_{1}\right)$ is the product of $\mathbb{P}^{1}$ and a double rational curve with 4 marked points.

- (The second fourfold contraction, following $\phi_{2}$.) Using again the birational contraction algorithm we can contract all the (product) surfaces over the fibers $g \in G$ : the resulting fourfold $Z$ is now elliptically fibered over $B$. The elliptic fibration admits a section away from the singular point in the base, where it becomes a double section. $Z$ is smooth outside 


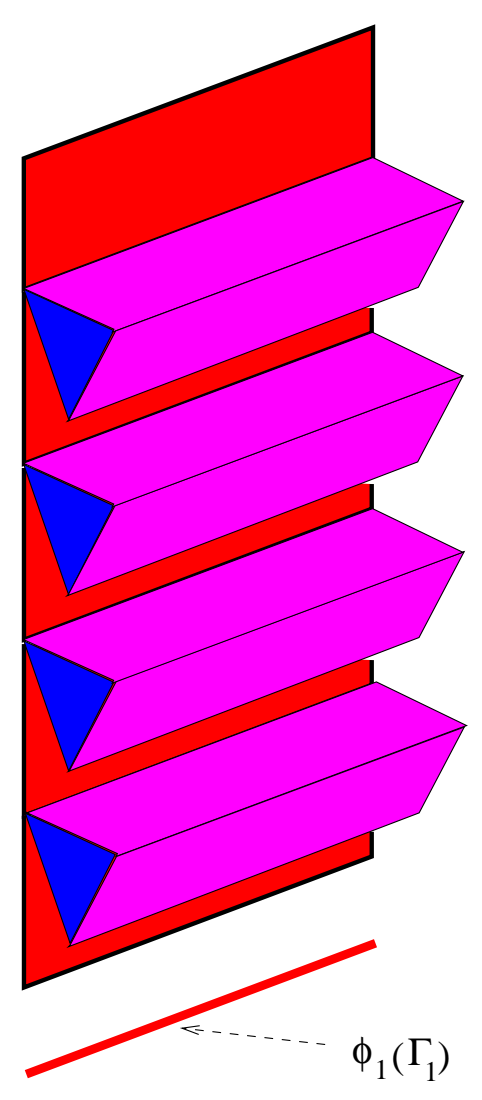

Fig. 16: The surface in $Z_{2}$ over $\phi_{1}\left(\Gamma_{1}\right)$ after the first contraction, following $\phi_{1}$.

the fiber over point $Q=\phi_{2} \cdot \phi_{1}\left(\Gamma_{1}\right)$ : over $Q$ there is a double rational curve with four terminal quotient singularities, as described in the previous section with the local quotient analysis.

\subsection{Two divisors with holomorphic Euler characteristic $1: \mathcal{E}_{31+} \subset Z_{+}$and $\mathcal{E}_{31} \subset Z$}

The fourfold birational transformation described in the previous section exchanges the two divisors $\mathcal{E}_{31+} \subset Z_{+}$and $\mathcal{E}_{31} \subset Z$; the first threefold is the smooth resolution of the second, which has terminal singularities. We can compute directly $\chi_{h}\left(\mathcal{E}_{31+}\right)=1$ and then deduce $\chi_{h}\left(\mathcal{E}_{31}\right)=1$.

In particular the flop among the fourfolds restricts to a birational morphism $\rho: \mathcal{E}_{31+} \rightarrow$ $\mathcal{E}_{31}$, which consists of the birational contraction of the reducible divisor $\pi_{+}^{*}\left(\Gamma_{+}\right)$to a double rational curve with four marked points, which are terminal singularities. This completes the proof that the (vertical) exceptional divisors in the singular fourfold also have holomorphic Euler characteristic 1. Moreover, since the exceptional divisors in the asymmetric resolution have $h^{0, i}\left(\mathcal{E}_{\bullet \bullet+}\right)=0, i=1,2,3$, it follows immediately that the same 
holds true for the exceptional divisors in the symmetric resolution and therefore they do contribute to the superpotential.

\subsection{Third cohomology of the exceptional divisors}

In this subsection we show that the third cohomology of the exceptional divisors is trivial, both for the smooth and singular Calabi-Yau fourfolds. Therefore, there is no theta function entering into the instanton prefactors. We recall that in the smooth resolution the exceptional divisors are either $\mathbb{P}^{1} \times d P_{9}$ or blow-ups of $\mathbb{P}^{1} \times d P_{9}$ along 8 (reducible) rational curves. It is clear that these are rational and that the third cohomology is trivial, $H^{3}\left(\mathcal{E}_{\bullet \bullet+}, \mathbb{Z}\right)=0$.

We would now like to prove that the same is also true for the exceptional divisors in the singular fourfold. The sequence of birational transformations taking one of the exceptional divisors in the smooth fourfold to the corresponding exceptional divisor in the singular fourfold is presented in the figure below.
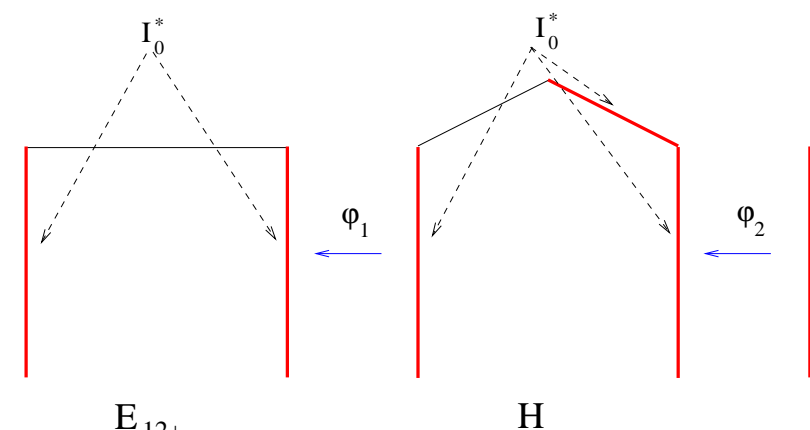

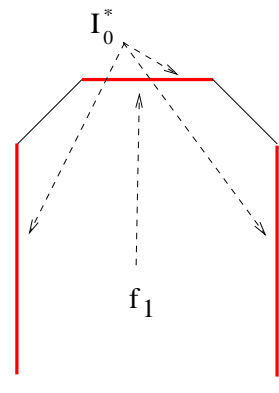

$\mathrm{H}_{1}$

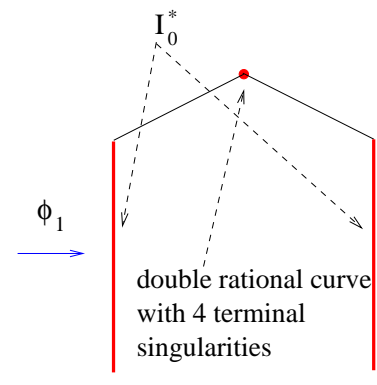

$\mathrm{E}_{12}$

Fig. 17: Sequence of birational transformations of the exceptional divisors.

A topological Euler characteristic computation shows that $H^{3}\left(\mathcal{H}_{1}, \mathbb{Z}\right)=0$, where $\mathcal{H}_{1}=\pi_{2+}^{*}\left(H_{1}\right)$. The group $H^{3}\left(\mathcal{E}_{12}, \mathbb{Z}\right)$ is nontrivial only if the relative homology group $H^{3}\left(\mathcal{H}_{1}, \pi_{2+}^{*}\left(f_{1}\right) ; \mathbb{Z}\right)$ is nontrivial. We have the following long exact sequence in homology

$\cdots \rightarrow H^{2}\left(\mathcal{H}_{1}, \mathbb{Z}\right) \stackrel{i^{*}}{\rightarrow} H^{2}\left(\pi_{2+}^{*}\left(f_{1}\right)\right) \rightarrow H^{3}\left(\mathcal{H}_{1}, \pi_{2+}^{*}\left(f_{1}\right) ; \mathbb{Z}\right) \rightarrow H^{3}\left(\mathcal{H}_{1}, \mathbb{Z}\right) \stackrel{i^{*}}{\rightarrow} H^{3}\left(\pi_{2+}^{*}\left(f_{1}\right)\right) \rightarrow \cdots$,

where $i: \pi_{2+}^{*}\left(f_{1}\right) \hookrightarrow \mathcal{H}_{1}$ is the inclusion. But $i^{*}: H^{2}\left(\mathcal{H}_{1}, \mathbb{Z}\right) \rightarrow H^{2}\left(\pi_{2+}^{*}\left(f_{1}\right), \mathbb{Z}\right)$ is a surjection, and therefore $H^{3}\left(\mathcal{H}_{1}, \pi_{2+}^{*}\left(f_{1}\right) ; \mathbb{Z}\right)$ is trivial. This proves that $H^{3}\left(\mathcal{E}_{12}, \mathbb{Z}\right)=0$ and this will be true for all the exceptional divisors in the Calabi-Yau fourfold $Z$ by symmetry. 


\section{Relation to Toroidal Orientifold Models}

At this point the world-sheet definition of our model should be clear - we take the supersymmetric sigma model with Calabi-Yau target space $Y$ and orientifold as discussed in $\S 2$, and then add background Neveu-Schwarz and Ramond-Ramond flux chosen to stabilize the complex structure and dilaton, as we will discuss in $\S 5$. While backgrounds with RR flux are not easy to handle in the NSR formalism, there is abundant evidence that the difficulties are just technical, and there is steady progress in developing formalisms which solve them [14].

While the model is well defined, it would be even better to obtain it starting from a solvable world-sheet definition, such as an orientifold of $T^{6}$. Unfortunately, we cannot expect to obtain our model in this way, for the simple reason that it contains wrapped branes which become massless in the orientifold limit. These are D3-branes wrapped on the exceptional two-cycles obtained by resolving the $\mathbb{C}^{3} / \mathbb{Z}_{2} \times \mathbb{Z}_{2}$ singularities; they give rise to BPS strings charged under RR two-forms, which become tensionless in this limit. Such states lead to divergences in the limit and the breakdown of world-sheet conformal field theory. Since we will be stabilizing the Kähler moduli away from this point, this is not a problem for our purposes, but it does preclude using the toroidal orientifold as the definition.

On the other hand, one can construct a very similar toroidal orientifold model which we will call $T^{\prime}$, suggested by Gopakumar and Mukhi [52] and first studied by Aldazabal et al [3]. In this section, we outline its construction, filling in some gaps in previous work, but leaving most of the details to future work. Besides explaining how the spectrum we found in F-theory arises, the main points we wish to see here are how the problematic states are given mass, and whether any variation of the construction might provide a solvable model.

The $T^{6} / \mathbb{Z}_{2}^{3}$ orientifolds were the first with $\mathcal{N}=1$ supersymmetry in $D=4$ to be constructed [16,12], and have been much studied since (see [5] for a review). Other important and relevant references on orientifold and orbifold compactification include $48,42,52,18,31,86,, 13,33,33,60,69,70,71,20]$.

We start with IIB string theory on $T^{6} \cong\left(T^{2}\right)^{3}$, with coordinates $z_{i}$ (with $i=1,2,3$ ) and complex structure moduli $\tau_{i}$ (so $z_{i} \cong z_{i}+1 \cong z_{i}+\tau_{i}$ ). Following [48,42], we then define an orientifold by choosing a set of Dirichlet branes, and then choosing an action of the orientifold group $\Gamma$ on both the compactification space and on the Chan-Paton factors of the branes. We will denote the orientation preserving subgroup of $\Gamma$ as $\Gamma_{0}$; the group $\Gamma$ is then an extension of $\Gamma_{0}$ by $\mathbb{Z}_{2}$. Let $\Gamma_{1}$ be the set of orientation reversing elements. 
Let $\Omega$ be the world-sheet orientation reversal, and $R_{i}$ be the reflection $z_{i} \rightarrow-z_{i}$, i.e.

$$
R_{1}:\left(z_{1}, z_{2}, z_{3}\right) \rightarrow\left(-z_{1}, z_{2}, z_{3}\right)
$$

resp. $R_{2}, R_{3}$.

The model $T^{\prime}$ is an orientifold of $\left(T^{2}\right)^{3}$ by $\Gamma \cong\left(\mathbb{Z}_{2}\right)^{3}$ with the generators

$$
g_{1}=\Omega R_{1} ; g_{2}=\Omega R_{2} ; g_{3}=\Omega R_{3} .
$$

The fixed sets of these group elements are $12 \mathrm{O} 7$ planes. The four planes fixed by $g_{i}$ are $z_{i}=0, z_{i}=1 / 2, z_{i}=\tau_{i} / 2$ and $z_{i}=\left(\tau_{i}+1\right) / 2$; let us denote the corresponding O7 planes as $\mathrm{O}_{i a}, \mathrm{O}_{i b}, \mathrm{O}_{i c}$, and $\mathrm{O}_{i d}$ respectively. Continuing, the group elements $g_{1} g_{2}, g_{2} g_{3}$ and $g_{3} g_{1}$ fix 48 lines of $\mathbb{C}^{2} / \mathbb{Z}_{2}$ orbifold points, while the group element $g_{1} g_{2} g_{3}$ fixes $64 \mathrm{O} 3$ planes. There are twelve groups of D7 branes, which will be taken coincident with the O7-planes, and denoted analogously as $\mathrm{D} 7_{i A}$ with $i=1,2,3$ and $A=a, b, c, d$. Finally, we will need D3 branes.

In further specifying the $\Gamma$ action, one must make several discrete choices. In the closed string sector, there are two such choices. First, one must choose an element $\epsilon$ in $H^{2}\left(\Gamma_{0}, U(1)\right)$, here $\mathbb{Z}_{2}$, usually called (Neveu-Schwarz) discrete torsion. The model $T^{\prime}$ and our model $T$ have $\epsilon$ trivial, i.e. no discrete torsion (in the conventions of [99]; some early references on this orientifold used the opposite convention). Second, one can modify the usual geometric actions by using symmetries which appear after twisting, say a symmetry which acts as -1 on all sectors twisted by a given $\mathbb{Z}_{2}$ and +1 otherwise, as discussed (for example) in [86] and used in the $T^{4} / \mathbb{Z}_{2} \times \mathbb{Z}_{2}$ orientifold of [18,31]. However, this is not required to define the models $T^{\prime}$ and $T$.

The closed string spectrum is obtained following standard techniques. In the untwisted sector, there is the $\mathcal{N}=1$ graviton multiplet; 7 chiral multiplets corresponding to the dilaton, 3 complex structure moduli and the 3 Kähler moduli $d z_{i} \wedge d \bar{z}_{i}$. The Kähler moduli are paired with RR scalars which transform as four-forms in $T^{6}$.

Twist sectors in a $D=6$ IIB orbifold without discrete torsion lead to $N=2$ hypermultiplets (and correspond geometrically to Kähler moduli). The $\Omega$ projection then keeps a single NS scalar and a single RR scalar in each of the 48 twist sectors. The hypermultiplet contained two RR scalars, one transforms as a scalar under the discrete symmetries of $T^{6}$; the other as a two-form; for example as $d z_{3} \wedge d \bar{z}_{3}$ in the $g_{1} g_{2}$ twist sector. The projections 
$g_{i}=\Omega R_{i}$ keep the two-form (which corresponds geometrically to a four-form integrated over an exceptional two-cycle).

The final spectrum is equivalent to that obtained by compactification on the smooth Calabi-Yau threefold with $h^{1,1}=51$ and $h^{2,1}=3$ discussed in $\S 2$. This would change if we modify the projections in the twisted sector, so we conclude that our models use the standard projection.

We next need to specify the $\Gamma$ action on the Chan-Paton factors for the Dirichlet branes. This is done by postulating matrices $\gamma(g)$ which form a projective representation of the orientifold group, in the following sense:

$$
\gamma(g) \gamma(h)=\epsilon(g, h) \gamma(g h) \quad g, h \in \Gamma_{0}
$$

for the orbifold elements, where $\epsilon(g, h)$ is a two-cocycle in $H^{2}\left(\mathbb{Z}_{2} \times \mathbb{Z}_{2}, U(1)\right) \cong \mathbb{Z}_{2}$ of discrete torsion. The orientifold elements satisfy

$$
\gamma(g) \gamma(u)=\epsilon^{\prime}(g, u) \gamma(g u) \quad g \in \Gamma_{0} ; u \in \Gamma_{1}
$$

and

$$
\gamma(u) \gamma(v)=\epsilon^{\prime \prime}(g, u) \gamma(v u) \quad u, v \in \Gamma_{1}
$$

where the factors $\epsilon^{\prime}$ and $\epsilon^{\prime \prime}$ are like cocycles, but must carry extra signs to cancel phases produced by the action $R(u)$ of orientation reversal on the world-sheet, as discussed in 48 and subsequent work.

The case which will concern us applies to open strings between pairs of branes with relative codimension 4 (in our models, all pairs $\mathrm{D} 7_{i}-\mathrm{D} 7_{j}$ with $i \neq j$ and all $\mathrm{D} 7_{i}$-D3 combinations). In this case, one can show that generally $R\left(\Omega R_{i}\right)^{2}=-1$, so that one must have $\gamma\left(\Omega R_{i}\right) \gamma\left(\Omega R_{i}\right)^{T}=-1$ for these open string sectors to be non-empty, which is required for consistency. For example, this is responsible for the $U S p$ gauge groups of D5 branes in the type I string in flat space; the combination $\gamma(\Omega)=1$ acting on D9 branes (leading to $S O(32)$ gauge symmetry) with $\gamma(\Omega)=i \sigma_{2} \otimes 1$ acting on D5 branes satisfies $\gamma \gamma^{T}=-1$. However, there is a loophole in this argument, as we discuss below.

One then projects the open strings of the underlying toroidal compactification as

$$
\begin{aligned}
\gamma(g) \phi \gamma(g)^{-1}=R(g) \phi ; & g \in \Gamma_{0} ; \\
\gamma(u) \phi^{t r} \gamma(u)^{-1}=-(R(g) \phi) ; & u \in \Gamma_{1} ;
\end{aligned}
$$

where $R(g)$ is the world-sheet action of the group element $g$. 
Finally, one must check the resulting theory for consistency; in particular tadpoles for unphysical gauge potentials sourced by space-time filling branes must cancel. In principle all such tadpoles can be seen as one-point functions on the disk and $\mathbb{R P}^{2}$ diagrams, but this check is usually done at one loop, as these diagrams (the torus, Klein bottle, Möbius strip and cylinder) have canonical normalizations (being traces over one string Hilbert spaces). Perhaps the clearest discussions of this related to the models at hand (actually, their double T-duals) appear in [5] and [71], which we refer to for details.

Given that our models do not have discrete torsion, we can start by considering linear (non-projective) representations of $\Gamma$. Since $\Gamma$ is abelian, the irreps are one-dimensional, each characterized by the action of the generators on the Chan-Paton factors,

$$
\gamma\left(g_{i}\right)=r_{i} ; \quad r_{i}= \pm 1
$$

Let us denote such an irrep as $R\left(r_{1}, r_{2}, r_{3}\right)$; we can think of each D-brane as transforming in one of these irreps.

We will then take 8 D7-branes in each group, transforming as

$$
\begin{array}{ll}
D 7_{1 A} & R(-1,+1,-1) \\
D 7_{2 A} & R(-1,-1,+1) \\
D 7_{3 A} & R(+1,-1,-1) .
\end{array}
$$

This leads to the gauge group $S O(8)^{12}$, and would seem to be in direct contradiction to the condition $\gamma\left(\Omega R_{i}\right) \gamma\left(\Omega R_{i}\right)^{T}=-1$ we discussed above.

The loophole which allows this was pointed out in 31,18 - while the rule $\Omega^{2}=-1$ applies to massless open strings, in some theories the massless sector is projected out. This is the case here; consider open strings between $\mathrm{D} 7_{1 A}$ and $\mathrm{D} 7_{2 B}$. The choice (4.3) implies that

$$
\gamma\left(g_{1} g_{2}\right) \phi_{12} \gamma\left(g_{1} g_{2}\right)^{-1}=-\phi_{12}
$$

and thus the massless strings in this sector are projected out. On the other hand, strings at half integral mass levels, for example $\psi_{-1 / 2}|0\rangle$, survive this projection. These get an extra sign from $g_{3}$ and thus we have

$$
R\left(g_{3}\right)^{2}=+1
$$

on this sector. Thus, one instead needs $\gamma\left(\Omega R_{i}\right) \gamma\left(\Omega R_{i}\right)^{T}=1$ as is satisfied by the representations $(4.2)$. 
The same considerations hold for all of the $7_{i}-7_{j}$ sectors with $i \neq j$, so there is no massless bifundamental matter in this theory. The massless $7_{i}-7_{i}$ adjoint matter is removed as well. For example, consider $X^{2}$ on $D 7_{1 A}$; the $g_{2}$ projection has the opposite sign from the $g_{1}$ projection, so together they project out the zero modes.

Another physical choice which is tied to this is the choice between "normal" or "exotic" $\mathrm{O}$ planes. The RR charge of an $\mathrm{O}$ plane can be inferred from the Möbius diagram with boundary on a D-brane $D_{i}$. This is proportional to

$$
\operatorname{Tr}_{i} \gamma(u) \gamma(u)^{T}
$$

for $u=\Omega R$, where $R$ is a group element fixing the plane $D_{i}$. If $\gamma(u)^{2}=1$, this correlates the sign of the $\mathrm{O}$ charge to the symmetry of the matrix $\gamma(u)$. A symmetric $\gamma(u)$ has negative charge and is a "normal" O plane, while antisymmetric $\gamma(u)$ has positive charge and is an "exotic" O plane. The corresponding projection leads to $S O$ and $S p$ gauge groups respectively for the corresponding D-branes. Of course, the gauge groups could be broken further by the other projections.

The choice (4.3) then corresponds to the choice of "normal" O7-planes in the model $T^{\prime}$. This also follows from tadpole cancellation; the $\mathrm{O}_{i A}$ plane tadpoles are each cancelled by the corresponding stack of $8 \mathrm{D} 7_{i A}$ branes.

The D7 branes also contribute to RR twisted tadpoles, one associated with each of the 48 fixed lines. Consider the $g_{1} g_{2}$ twisted tadpoles; these receive contributions from branes extended along $z_{3}, \mathrm{D} 7_{1 A}$ and $\mathrm{D} 7_{2 A}$, proportional to $\gamma\left(g_{1} g_{2}\right)$. One can check that in (4.3), $\operatorname{Tr} \gamma\left(g_{1} g_{2}\right)=\operatorname{Tr} \gamma\left(g_{1}\right) \gamma\left(g_{2}\right)=0$, and that the other tadpoles cancel as well. It is this condition which forces the D7's to be taken in groups of 8 and thus the $S O(8)^{12}$ gauge group.

This leaves the D3 tadpole. The $64 \mathrm{O} 3$ planes contribute through the $\mathbb{R P}^{2}$ worldsheet diagram constructed using the $g_{1} g_{2} g_{3}$ identification. One can also check that the D7 branes do not contribute to this tadpole. This suggests that the total result for the tadpole is the same as what would be obtained in the $T^{6} / \mathbb{Z}_{2}$ orientifold dual to type I theory, and will cancel if $\operatorname{Tr}_{D 3} \gamma(1)=32$, i.e. if we add $32 \mathrm{D} 3$ branes in some $\Gamma$ representation.

However, to get a model with an F-theory dual, we must take the D3 branes in a regular representation. Then, massless open $3-7$ strings will survive the projection, and $\Omega^{2}=-1$ in the $3-7$ sector. This will require us to take $\gamma\left(\Omega R_{1} R_{2} R_{3}\right)$ antisymmetric, leading to $U S p$ gauge groups on the D3 branes, and again corresponding to exotic O3 planes. These 
contribute to the tadpole with the opposite sign, and thus the tadpole cannot be cancelled with D3-branes. One can obtain a consistent model by using anti-D3-branes, now with $\operatorname{Tr}_{\text {anti D3 }} \gamma(1)=32$.

Such a result is known from the world-sheet analysis of these orientifolds [4, 71] and actually can be seen just by considering factorization of the Klein bottle amplitude. In fact, the consistency condition found there is weaker. Let $\epsilon_{i}$ be the types of the $\mathrm{O} 7_{i}$ planes ( $\epsilon_{i}=-1$ is normal and $\epsilon_{i}=+1$ is exotic) and $\epsilon_{0}$ be the type of the O3 plane. Then, taking $\epsilon_{3}=-1$ to define the conventions of charge, one must have

$$
\epsilon=\epsilon_{0} \epsilon_{1} \epsilon_{2}
$$

Thus, with our present choices $\epsilon=+1$ and $\epsilon_{1}=\epsilon_{2}=-1$, we are forced to take exotic O3 planes with $\epsilon_{0}=+1$. Their tadpole can be cancelled with 32 anti D3-branes, to obtain model $T^{\prime}$.

Another way to satisfy the constraint (4.4) is to take $\epsilon_{0}=-1$ (normal O3 planes) with $\epsilon=-1$ (non-trivial discrete torsion). However, one cannot make the choices (4.3) in this case, as taking $S O$ groups for both the D3's and D7's conflict with the $\Omega^{2}=-1$ constraint. It is argued in [12,71] that the only model solving these constraints is the one found in [12,16] with $U S p(16)^{4}$ gauge group.

Finally, another possibility which may be consistent (we have not checked this in all detail) would be to choose the D3 branes in the fourth representation $R(+1,+1,+1)$, which would kill the $3-7$ matter and lead to gauge group $S O(8)^{16}$. This model, $T^{\prime \prime}$, is in many ways the most symmetric, though it does not have an F-theory dual.

Let us conclude by describing the world-volume theory of the anti-D3 branes in theory $T^{\prime}$, as it has some interesting features, especially in how its moduli space reproduces the resolved Calabi-Yau geometry discussed in $\S 2$. The $\Gamma$ representation for these branes is 4 copies of a projective version of the regular representation, which acts by group multiplication on an 8 dimensional Hilbert space $\mathcal{H} \cong \mathbb{C} \Gamma$ with basis $\Gamma$,

$$
\gamma(g)|h\rangle=\rho(g, h)|g h\rangle
$$

with a cocycle $\rho(g, h)$ defined by $\rho\left(\Omega^{p} g_{0}, \Omega^{q} h_{0}\right)=(-1)^{p q}$. Equivalently, it is 4 copies of the regular representation of $\Gamma_{0}$ tensored with $\gamma(\Omega)=i \sigma_{2} \equiv \sigma$, so that the resulting gauge group is $U S p(8)^{4}$ (where 8 is the dimension of the fundamental representation). We will subsequently denote the different gauge factors as $U S p\left(N_{i}\right)\left(\right.$ all $\left.N_{i}=8\right)$. 
As anti-D3-branes in Calabi-Yau compactification break supersymmetry, we must consider the fermions separately. From the world-sheet point of view, an anti-D3 can be defined by reversing the GSO projection; this changes the sign of the $\Omega$ projection (4.1) on the fermions and replaces the adjoint representation with the representation with the opposite symmetry, for $U S p$ the antisymmetric representation.

The rest of the anti-D3 and anti-D3-D7 open string spectrum can be derived along the lines of [3]. We can start with the usual probe theory of the $\mathbb{C}^{3} / \mathbb{Z}_{2} \times \mathbb{Z}_{2}$ singularity [56], an $\mathcal{N}=1 U(N)^{4}$ theory with 12 bifundamental chiral multiplets, one $\left(\bar{N}_{i}, N_{j}\right)$ for each $1 \leq i \neq j \leq 4$, denote these $Z_{i j}$. To obtain theory $T^{\prime}$, we apply the $\Gamma_{1}$ projection, which sets

$$
Z_{i j}=\sigma Z_{j i}^{t} \sigma
$$

(with $\sigma \equiv i \sigma_{2}$ ) for both fermions and bosons, to obtain six complex bosons and Weyl fermions in the $\left(N_{i}, N_{j}\right)$ for $1 \leq i<j \leq 4$.

Substituting these projected fields into the potential and Yukawa couplings of the underlying $\mathcal{N}=1$ theory, one obtains a Lagrangian with a non-trivial potential. Perhaps the most interesting case of this is the fate of the D-terms of the $\mathcal{N}=1$ theory, which were

$$
V_{D}=\sum_{i=1}^{4} \operatorname{tr}\left(\zeta_{i} \cdot 1-\sum_{j \neq i} Z_{i j} Z_{i j}^{\dagger}\right)^{2}
$$

where $\zeta_{i}$ are four Fayet-Iliopoulos parameters controlled by the Kähler moduli as in [42], and satisfying $\sum_{i=1}^{4} \zeta_{i}=0$. These terms also survive the projection, leading to a potential quadratic in the combinations $H_{i j}=Z_{i j} Z_{i j}^{\dagger}$. Most importantly, it depends on the $\zeta_{i}$, as must be the case for the resulting moduli space to depend on the Kähler moduli. This would have been impossible for an $\mathcal{N}=1$ supersymmetric probe theory with $U S p$ gauge groups, as Fayet-Iliopoulos terms are not possible for semisimple groups.

To find the moduli space of the resulting theory, we can again start from the analysis of the underlying $\mathbb{C}^{3} / \mathbb{Z}_{2} \times \mathbb{Z}_{2}$ probe theory, and then study the effect of the orientifold quotient. In [56], it is shown that the $U(1)^{4}$ theory of a single probe indeed reproduces the two resolutions of $\mathbb{C}^{3} / \mathbb{Z}_{2} \times \mathbb{Z}_{2}$ discussed in $\S 2$, depending on the signs of the FayetIliopoulos parameters. Since the potential was preserved by the constraints, a set of 8 such configurations satisfying (4.6) will be a solution of the $T^{\prime}$ anti-D3 theory. Furthermore, the orientifold action $z_{i} \rightarrow-z_{i}$ on each anti-D3 is realized as the gauge transformation $Z_{i j} \rightarrow \sigma_{2} Z_{i j} \sigma_{2}^{-1}$ in its $U S p(2)^{4}$ subgroup. 
To complete the theory, the anti-D3-D7 bosons transform as scalars under rotations in the dimensions transverse to the D7, while the fermions transform non-trivially. Taking this into account, one should find complex bosons $\phi_{i}$ in the $\left(8_{i}, N_{i}\right)$, one for each of the three of the $U S p\left(N_{i}\right)$ factors $i=1,2,3$ which carry the same representations appearing in (4.3), one fermions in the $\left(8_{i}, N_{i}\right)$ for $i=1,2,3$, and three fermions in the $\left(8_{i}, N_{4}\right)$. This spectrum allows for mass terms for all 3-7 strings proportional to the 3-7 distance parameters $\left|Z_{i j}\right|$, the bosons as $Z_{i j} Z_{i j}^{\dagger}\left|\phi_{i A}\right|^{2}$ and the fermions as $\psi_{A i} Z_{i 4} \psi_{A 4}$.

From this discussion, another way to search for variations on the orientifold which would have negative D3 tadpole and thus admit supersymmetric flux vacua would be to look for a candidate supersymmetric probe theory whose moduli space is the orientifold. While this can be done in the strict orientifold limit $\left(\zeta_{i}=0\right)$ and even with one non-zero FI parameter [84], after some attempts, we suspect there is no weakly coupled supersymmetric probe theory which realizes this moduli space with all three of the resolution parameters.

Indeed, a similar result was already found in six dimensions by [2], who found difficulties in constructing a supersymmetric probe theory for the orientifold of [18, 31]. That orientifold is closely related to what one obtains by moving away from one D7 plane here, say $z_{3} \sim 0$, so perhaps an anti D3-brane probe is also the appropriate probe there. More generally, it would be interesting to have a simple argument for why certain orientifold geometries can be obtained from antibrane probes and not brane probes.

The upshot of this discussion is that, while we can define consistent $T^{6} / \mathbb{Z}_{2} \times \mathbb{Z}_{2}$ orientifolds containing the $S O(8)^{12}$ gauge group predicted by the F-theory construction, we have not found a construction of the model $T$ with D3 tadpole -28 discussed in $\S 2$; instead we have a model $T^{\prime}$ with D3 tadpole +4 cancelled by anti D3-branes with gauge group $U S p(8)^{4}$, and another model $T^{\prime \prime}$ with D3 tadpole -4 cancelled by "fractional" D3branes with gauge group $S O(8)^{4}$ and no matter (we have not fully checked its consistency however).

One might attempt to start with the second of these models, and replace the D3-branes with flux, to obtain a fully stabilized model from a perturbative world-sheet starting point. This may be possible, but one needs to stabilize the 51 complex structure moduli of the orbifold with discrete torsion (and the dilaton) and obtain small $\left|W_{0}\right|$ using only 4 units of flux. This seems very optimistic, though not obviously impossible. Other tricks might help, for example increasing the flux tadpole by adding $D 9-\bar{D} 9$ pairs along the lines of [20,78. 
As we said in the beginning, the failure to find the model $T$ as a toroidal orientifold is not entirely surprising (in retrospect) as it has massless nonperturbative states at the orientifold point. Conversely, the orientifold CFT's incorporate discrete choices which give mass to all of these states, either exotic $\mathrm{O}$ planes or lack of vector structure [92 forcing $B \neq 0$, or discrete torsion. While we see no argument which in principle forbids the existence of a toroidal or CFT orientifold with flux stabilizing all moduli, for the models at hand these choices decrease the D3 tadpole to where such vacua are few or non-existent.

\section{Complex Structure Stabilization}

The fourfold $Z$ we are studying has $h^{3,1}(Z)=4$ - in IIB language, the Calabi-Yau manifold $Y$ contributes three complex structure moduli, and the fourth modulus is identified with the axio-dilaton $\phi$. Achieving moduli stabilization in a controlled manner in the spirit of [65] requires a small number $e^{K}|W|^{2}$, where $W \sim \int_{Y}(F-\phi H) \wedge \Omega$ is the Gukov-Vafa-Witten superpotential [59]. Therefore, we proceed in this section to outline the construction of explicit flux vacua in this model, that provide the small number. It should be quite clear that a similar strategy could be applied in more general models, the only difficulty being that as the number of complex moduli increases, explicit computations become difficult. In fact, other examples of explicit flux vacua with small $e^{K}|W|^{2}$ $\left(\sim 10^{-3}\right.$ and less) were explicitly constructed in [51, 37], and the statistical results suggest that much smaller values are attainable [35], with the fraction of vacua having $e^{K}|W|^{2} \leq \epsilon$ scaling like $\epsilon$.

Before blowing up the singularities of the base, its periods are (up to simple issues of normalization) just a subset of those of the $T^{6} / \mathbb{Z}_{2}$ orientifold. Resolving the space does not change the periods of the holomorphic three-form. Therefore, we can use any flux vacua found in the $T^{6} / \mathbb{Z}_{2}$ orientifold (which has been studied in some detail in e.g. [64, 38]), subject to the constraints that:

(1) The total D3 charge in the fluxes, on the covering space of the orientifold, should satisfy:

$$
N_{\text {flux }}=\frac{1}{(2 \pi)^{4}\left(\alpha^{\prime}\right)^{2}} \int_{Y} H \wedge F \leq 56 .
$$

Any difference can be compensated by adding D3 branes. We shall actually choose to saturate the tadpole condition entirely with flux in our most explicit example.

One can understand the appearance of the 56 as follows. We have taken the integral over all of $Y$; the integral over the orientifold would reduce this by a factor of 2 , yielding 
the correct tadpole charge of 28 , as derived in $\S 2.4$. However, for further ease, we can relate the condition (5.1) to one formulated on the $T^{6}$ covering space of the orbifold $Y=T^{6} / \mathbb{Z}_{2} \times \mathbb{Z}_{2}$ as well. If we choose a standard basis $C_{i}$ for $H^{3}\left(T^{6}, \mathbb{Z}\right)$ with e.g. $C_{1}=d x^{1} \wedge d x^{2} \wedge d x^{3}$ and so forth, then a symplectic integral basis for $H^{3}(Y, \mathbb{Z})$ is given by considering $B_{i}=2 C_{i}$ (for those forms which are projected in only, of course). Then $\int_{Y} B_{i} \wedge B_{j}=\frac{1}{4} \int_{T^{6}} 4 C_{i} \wedge C_{j}=\int_{T^{6}} C_{i} \wedge C_{j}$. So in fact, we can easily translate any flux vacua satisfying

$$
\frac{1}{(2 \pi)^{4}\left(\alpha^{\prime}\right)^{2}} \int_{T^{6}} H \wedge F \leq 56
$$

on $T^{6}$, with fluxes in the $C_{i}$ basis, to flux vacua on the orbifold which also satisfy the necessary tadpole condition.

An important caveat is that if we wish to avoid the need to introduce exotic O3 planes, which contribute differently to the tadpole than standard O3 planes, we should use only even fluxes in the construction [47]. We will adhere to the use of even fluxes in our examples; it is quite possible that more general configurations with odd fluxes and exotic planes would also yield interesting models.

(2) We can only turn on the fluxes in the covering space, which are consistent with the orbifold action. This is an 8-parameter family of choices for both $H$ and $F$, where one is allowed to turn on fluxes in cohomology classes with precisely one leg along each $T^{2}$ in the $T^{6}$. In fact, for our explicit example, we will restrict to the even smaller subclass where

$$
\begin{aligned}
F= & a^{0} d x^{1} \wedge d x^{2} \wedge d x^{3}+a\left(d x^{2} \wedge d x^{3} \wedge d y^{1}+d x^{3} \wedge d x^{1} \wedge d y^{2}+d x^{1} \wedge d x^{2} \wedge d y^{3}\right) \\
& -b\left(d y^{2} \wedge d y^{3} \wedge d x^{1}+d y^{3} \wedge d y^{1} \wedge d x^{2}+d y^{1} \wedge d y^{2} \wedge d x^{3}\right)+b_{0} d y^{1} \wedge d y^{2} \wedge d y^{3}
\end{aligned}
$$

and with similar integer choices $c^{0}, c, d, d_{0}$ defining $H$. As explained above, we will choose only even integers here to avoid the subtleties explained in [47].

The flux vacua of class (5.3) were studied in [64] and in fact completely classified in [38] (while powerful analytical results which are applicable both to this model and to a wider class were also derived in 81, where the connection between flux vacua and the attractor mechanism was exploited). It is a simple matter to do a computer search at fixed $N_{\text {flux }}$ to find vacua of this form with small $g_{s}$ and moderately small $e^{K}|W|^{2}$. Here we present two examples:

7 At least for A. Giryavets, whom we thank for significant help! 
Example A): At the relevant value $N_{\text {flux }}=56$, the explicit flux choice

$$
\left(a^{0}, a, b, b_{0}\right)=(0,10,-10,28), \quad\left(c^{0}, c, d, d_{0}\right)=(2,2,-2,4)
$$

yields an interesting vacuum for our purposes. It saturates the entire tadpole constraint with flux, so no mobile space-filling D3s need be introduced. The resulting complex structure is of the form $\left(T^{2}\right)^{3}$ with all two-tori sharing the same modular parameter; explicitly

$$
\tau=.46+.84 i, \quad \phi=7+3.64 i
$$

Using the $S L(2, \mathbb{Z})$ S-duality symmetry one can easily shift $\phi$ into the fundamental domain; the resulting string coupling is $g_{s} \sim .27$. The value of $e^{K}|W|^{2}$ in this vacuum is 0.100 , in units where $(2 \pi)^{2} \alpha^{\prime}=1$.

Example B): Also at $N_{\text {flux }}=56$, the choice

$$
\left(a^{0}, a, b, b_{0}\right)=(0,8,8,28), \quad\left(c^{0}, c, d, d_{0}\right)=(2,0,0,2)
$$

is interesting. The resulting vacuum has $g_{s}=.21$ and $e^{K}|W|^{2} \sim .350$.

Both examples have only moderately small tuning parameter $e^{K}|W|^{2}$. We shall see in $\S 6.5$ that with these values of $g_{s}$ and the gravitino mass, one can nevertheless argue that the leading potential (after including Euclidean D3 instantons and gaugino condensates), which exhibits stabilization of all moduli, receives only rather small corrections from the known higher order effects.

We note that following the estimates of [6. [35], this model actually has $\mathcal{O}\left(10^{13}\right)$ flux vacua, and most likely yields a minimal $e^{K / 2}|W| \sim 10^{-5}$. By generalizing our ansatz somewhat, we could therefore improve the values of $g_{s}$ and $e^{K}|W|^{2}$ even in this example with few complex moduli. Of course in models with larger numbers of complex structure moduli, the number of flux vacua is vastly larger, and the tuning parameter should correspondingly attain smaller values.

\section{Kähler Moduli Stabilization}

Kähler moduli can be stabilized by nonperturbative contributions to the superpotential, as outlined in [65]. There are two sources of such contributions: gaugino condensation and IIB Euclidean D3-brane instantons wrapping suitable divisors. In the M-theory 
picture, both effects are associated to the existence of divisors of holomorphic Euler characteristic 1 in the fourfold [101, 67, 8

In the following we will show in detail that these effects do indeed fix the Kähler moduli in a controlled regime in our model. Essentially the idea is that the 48 exceptional divisors are rigid, so they give $48 \mathrm{D} 3$ instanton contributions to $W$, and the $S O(8)^{12}$ gauge theory has no matter, so this produces 12 gaugino condensation contributions. Together this gives enough independent terms in the superpotential to fix all Kähler moduli. If $W_{\text {flux }}$ is sufficiently small (in fact as we will see just below order $\mathcal{O}(1)$ is already sufficient in our model), the radii will be stabilized at values such that $\alpha^{\prime}$ corrections are small. Moreover, in this regime the effect of Kähler moduli variations on the complex structure moduli is exponentially suppressed, justifying integrating out the former first and treating Kähler moduli stabilization separately.

\subsection{Divisors}

As discussed in sections 2 and 3, there are 51 Kähler moduli, 3 of which descend from the radii of $B=Y / \mathbb{Z}_{2}=\left(\mathbb{P}^{1}\right)^{3}$ we have before blowing up, and 48 from blowing up the fixed lines. The moduli space is holomorphically parametrized by complexified divisor volumes

$$
\tau_{n}=\frac{1}{\ell_{s}^{4}} \int_{D_{n}} d V+i C_{4} .
$$

Here $\ell_{s} \equiv 2 \pi \sqrt{\alpha^{\prime}}$, and we work in ten dimensional Einstein frame. Our normalization conventions are described in detail in appendix A. Unless otherwise specified, we will work directly in the quotient $B=Y / \mathbb{Z}_{2}$. A suitable basis of divisors is given by $\left\{R_{i}, E_{i \alpha, j \beta}\right\}$, where $i, j=1,2,3$ and $\alpha, \beta=1, \ldots, 4$. The $R_{i}$ are "sliding" divisors, descending from the $\left(\mathbb{P}^{1}\right)^{2} \subset\left(\mathbb{P}^{1}\right)^{3}$, and essentially given by $z_{i}=$ const.. The $E_{i \alpha, j \beta}(i<j)$ are the exceptional divisors obtained by blowing up the fixed line at the intersection of $z^{i}=z_{\alpha}^{\mathrm{fix}}$ and $z^{j}=z_{\beta}^{\mathrm{fix}}$, where the $z_{\alpha}^{\text {fix }}$ label the four fixed points of $T^{2} / \mathbb{Z}_{2}$.

The D7-brane stacks wrap the divisors obtained from the fixed planes $z^{i}=z_{\alpha}^{\mathrm{fix}}$ after blowing up. In line with the notations of section 2 and 3 , we denote these by $D_{i \alpha}$. The difference the sliding divisors $R$ and these 7-brane divisors $D$ is given by the sum

\footnotetext{
8 In certain circumstances, divisors of $\chi_{h}>1$ can contribute [53,66, 98. The precise criteria for this to occur are still being determined, and we will see in $\S 6$ that any such contributions can be self-consistently neglected in our example.
} 
of the exceptional divisors $E$ obtained from the fixed lines inside the fixed plane under consideration (see section 2 and below for more discussion). For example

$$
D_{1 \alpha}=R_{1}-\sum_{\beta} E_{1 \alpha, 2 \beta}-\sum_{\gamma} E_{3 \gamma, 1 \alpha}
$$

This relation is independent of the chosen resolution.

The contribution to the superpotential from a D3 instanton wrapping an exceptional divisor $E_{i \alpha, j \beta}$ is

$$
\Delta W \sim e^{-2 \pi \tau_{i \alpha, j \beta}}
$$

while gaugino condensation in the $S O(8)$ gauge theory associated to the divisor $D_{i \alpha}$ contributes

$$
\Delta W \sim e^{-2 \pi \tau_{i \alpha} / 6}
$$

which can be expressed in terms of the basis coordinates using (6.2). Here we used that $c_{2}(S O(8))=6$. We will discuss these contributions and various subtleties in much more detail further on.

\subsection{Kähler potential}

After integrating out the complex structure moduli, the classical Kähler potential is given by

$$
K=K_{0}-2 \log V
$$

where $K_{0}$ is a constant (the contribution to $K$ coming from the complex structure part) and $V$ is the volume of the threefold $B$ in Einstein frame and in units of $\ell_{s}=2 \pi \sqrt{\alpha^{\prime}}$.

Denoting our basis of divisors collectively by $\left\{S_{a}\right\}, a=1, \ldots, 51$, and expanding the Kähler form as $J=y^{a} S_{a}$, the volume is

$$
V=\frac{1}{6} S_{a b c} y^{a} y^{b} y^{c}
$$

where $S_{a b c} \equiv S_{a} \cdot S_{b} \cdot S_{c}$ is the triple intersection product of the divisors.

To compute these triple intersection numbers, we proceed as in the local model of section 2. First we deduce the triple intersection numbers between distinct divisors from the (overcomplete) set $\left\{S_{A}\right\}_{A=1}^{63}=\left\{R_{i}, E_{i \alpha, j \beta}, D_{i \alpha}\right\}$. This can be done using a local toric model. Then we compute the triple intersections with two and three equal divisors, i.e. $S_{A A B}$ and $S_{A A A}$, using the 12 linear relations (such as (6.2)) between the $S_{A}$. These are 
of the form $r^{A} S_{A}=0$, and thus give $63 \times 63 \times 12$ equations of the form $S_{A B C} r^{C}=0$. The number of independent equations (far) exceeds the number of unknowns, so solving them determines all $S_{A A B}$ and $S_{A A A}$, and gives a nontrivial consistency check of the input of step 1 . The system is sparse, so it can be solved very fast using a computer.

The reason we can use a local model for step 1 is that a triple intersection involving indices $i \alpha$ and $i \alpha^{\prime}$ with $\alpha^{\prime} \neq \alpha$ will automatically be zero, because the corresponding two divisors are manifestly disjoint (this is clear from fig. 8). Thus to compute intersections we can fix $(1 \alpha, 2 \beta, 3 \gamma)$ and model the local geometry near $z^{i}=\left(z_{\alpha}^{\mathrm{fix}}, z_{\beta}^{\mathrm{fix}}, z_{\gamma}^{\mathrm{fix}}\right)$ by $\mathbb{C}^{3}$ blown up along three lines meeting at the origin, as described in section 2 and 3 . To model also the sliding divisors, we compactify this geometry to $\left(\mathbb{P}^{1}\right)^{3}$ blown up along three lines, where the divisors at infinity model the sliding divisors $R_{i}$. Toric methods can now be used to derive the triple intersections between distinct divisors. This depends on the chosen resolution, asymmetric or symmetric. Both cases are presented in the figure below.

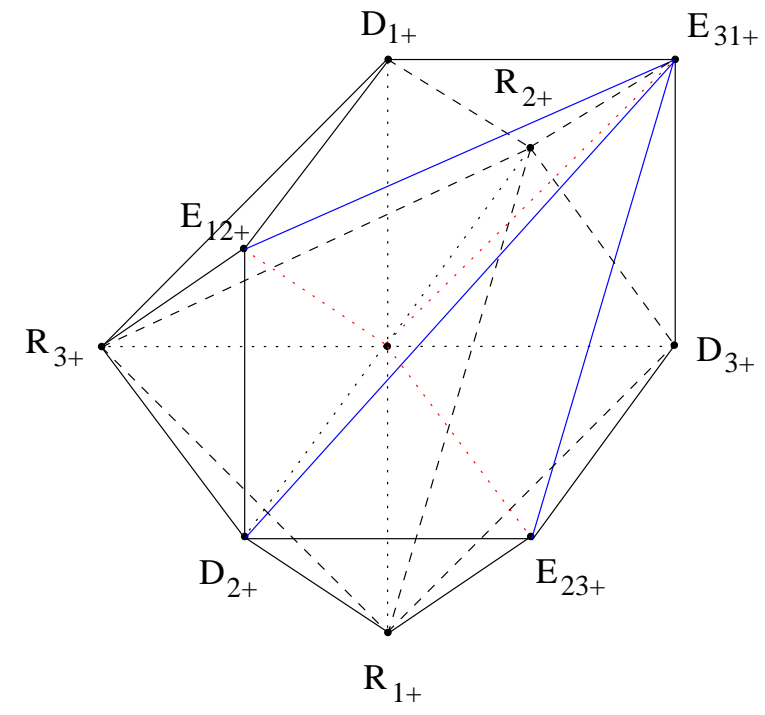

(a)

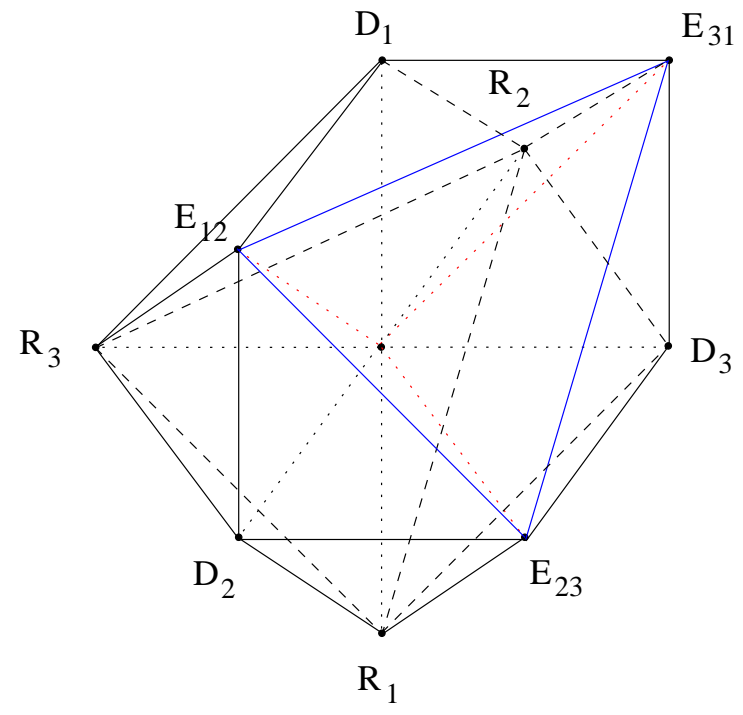

(b)

Fig. 18: $\left(\mathbb{P}^{1}\right)^{3}$ blown-up along three lines that meet in a point. (a) Asymmetric and (b) symmetric triangulation of the fan.

Recall that divisors are represented as points (or rather rays from the origin to these points), with lines between pairs of points representing intersections, and triangles representing triple intersections. All such triple intersection products equal 1, with the exception of $E_{12} \cdot E_{23} \cdot E_{31}=1 / 2$ in the symmetric resolution because of the $\mathbb{Z}_{2}$ singularity at the intersection point. This is in agreement with (2.9). 
This data suffices to compute all triple intersection products. Parametrizing the Kähler form as

$$
J=r_{i} R_{i}-t_{1 \alpha, 2 \beta} E_{1 \alpha, 2 \beta}-t_{2 \beta, 3 \gamma} E_{2 \beta, 3 \gamma}-t_{3 \gamma, 1 \alpha} E_{3 \gamma, 1 \alpha}
$$

gives the following expression for the volume $V=J^{3} / 6$ :

$$
\begin{aligned}
V_{\mathrm{symm}}= & r_{1} r_{2} r_{3}-\frac{1}{2}\left(r_{1} \sum_{\beta \gamma} t_{2 \beta, 3 \gamma}^{2}+\cdots\right)-\frac{1}{3}\left(\sum_{\alpha \beta} t_{1 \alpha, 2 \beta}^{3}+\cdots\right) \\
& +\frac{1}{4}\left(\sum_{\alpha \beta \gamma} t_{1 \alpha, 2 \beta} t_{2 \beta, 3 \gamma}^{2}+t_{1 \alpha, 2 \beta} t_{3 \gamma, 1 \alpha}^{2}+\cdots\right)-\frac{1}{2} \sum_{\alpha \beta \gamma} t_{1 \alpha, 2 \beta} t_{2 \beta, 3 \gamma} t_{3 \gamma, 1 \alpha}
\end{aligned}
$$

if everything is resolved symmetrically, and

$$
\begin{aligned}
V_{\text {asymm }}= & r_{1} r_{2} r_{3}-\frac{1}{2}\left(r_{1} \sum_{\beta \gamma} t_{2 \beta, 3 \gamma}^{2}+\cdots\right)-\frac{2}{3}\left(\sum_{\alpha \beta} t_{1 \alpha, 2 \beta}^{3}+\sum_{\beta \gamma} t_{2 \beta, 3 \gamma}^{3}\right) \\
& +\frac{1}{2}\left(\sum_{\alpha \beta \gamma} t_{3 \gamma, 1 \alpha} t_{1 \alpha, 2 \beta}^{2}+t_{3 \gamma, 1 \alpha} t_{2 \beta, 3 \gamma}^{2}\right)
\end{aligned}
$$

if everything is resolved asymetrically with distinguished direction $i=2$. Here "+ $\cdots "$ means adding $(1,2,3)$ cyclically permuted terms. Many other mixed symmetric/asymmetric resolutions are possible of course, but we will restrict to these two.

As explained in the previous subsection, the proper holomorphic coordinates on moduli space are the complexified divisor volumes, rather than complexified $y^{a}$. The divisor volumes are given in terms of the latter by

$$
V_{a}=\partial_{y^{a}} V=\frac{1}{2} S_{a b c} y^{b} y^{c}
$$

It is not possible in practice to invert these relations explicitly, but fortunately this is not necessary to find critical points.

We will discuss corrections to the Kähler potential below.

\subsection{Curve areas and Kähler cone}

Let $\left\{C_{r}\right\}$ be a basis of the Mori cone, i.e. the cone in $H_{2}(B, \mathbb{Z})$ of effective holomorphic curves. The geometric moduli space for a fixed resolution is given by the dual cone in $H^{2}(B, \mathbb{R})$, i.e. the space of Kähler forms $J$ for which the curve areas $A_{r} \equiv C_{r} \cdot J>0$. This is the Kähler cone. 
When finding critical points of $W$, it is important to verify that they actually lie in the Kähler cone. To do this, we need a set of generators $C_{r}$ for the Mori cone, or equivalently their areas $A_{r}$ as a function of the coordinates $r_{i}, t_{i \alpha, j \beta}$. We used the algorithm outlined in appendix B of [37], which is essentially the procedure followed for the local model in section 2, with the following results. For the symmetric resolution:

$$
\begin{aligned}
A_{i, j \beta} & =r_{i}-\sum_{\alpha} t_{i \alpha, j \beta} \\
A_{\alpha \beta \gamma}^{++-} & =\frac{1}{2}\left(t_{1 \alpha, 2 \beta}+t_{2 \beta, 3 \gamma}-t_{3 \gamma, 1 \alpha}\right),
\end{aligned}
$$

plus cyclic permutations +-+ and -++ of the latter. The curves of the second line are exceptional curves produced by the blowup and will be small near the orbifold point, while those of the upper line are related to the $\mathbb{P}^{1} \subset\left(\mathbb{P}^{1}\right)^{3}$ we have before blowing up and stay finite in the orbifold limit. Note that the $A_{\alpha \beta \gamma}$ curves are nothing but the exceptional curves $\widetilde{C}_{i}$ of the local model $X / \mathbb{Z}_{2}$ discussed in section 2 . For the asymmetric resolution we have

$$
\begin{aligned}
A_{i, j \beta} & =r_{i}-\sum_{\alpha} t_{i \alpha, j \beta} \quad \text { except } \quad(i, j)=(1,2) \text { and }(3,2) \\
A_{\alpha \beta \gamma} & =-t_{1 \alpha, 2 \beta}-t_{2 \beta, 3 \gamma}+t_{3 \gamma, 1 \alpha} \\
A_{1 \alpha, 2 \beta} & =t_{1 \alpha, 2 \beta} \quad \text { and } \quad A_{2 \beta, 3 \gamma}=t_{2 \beta, 3 \gamma} .
\end{aligned}
$$

\subsection{Superpotential}

The contributions to the superpotential from D3 instantons wrapping the exceptional divisors are of the form (6.3), and those from gaugino condensation of the form (6.4). We now discuss these in more detail.

We start with the D3 instantons. They wrap the exceptional divisors, which as discussed in section 2 and 3 have $h^{i, 0}=0$ for $i>0$. This is true both for the divisor in the threefold as well as for its lift to the M-theory fourfold $\widetilde{Z}$ (where the D3 becomes an M5). Therefore, these instantons do give contributions to the superpotential [101]. Moreover, each such divisor is unique in its homology class, so there can't be cancellations between different divisors in the same homology class.

The full M5-brane instanton partition function also involves a theta function, essentially from summing over worldvolume 3-form fluxes $h$ on the M5 [103,82, This could lead to a problematic suppression of the instanton amplitude by a factor $e^{-c / g_{s}}$, or even to com-

plete cancellation between contributions at different values of $h$. A similar phenomenon is 
observed for D3 instantons. 9 Fortunately, in our case, as argued in section 2.3 and proven in section 3.8, the third cohomology of the M5 divisors is trivial, so there is no sum over $h$, and this problem does not occur. From the IIB point of view (related by straightforward KK reduction on the elliptic fiber) the sum is absent because the $\mathbb{Z}_{2}$-twisted second cohomology group of the D3 divisor is trivial.

Additional zeros of the partition function can also arise when mobile D3-branes are present or when $h^{2,1}$ of the fourfold is nonzero [49], but since we saturate the tadpole by fluxes, there are no D3-branes, and as we saw in section 2 and $3, h^{2,1}(\widetilde{Z})=0$.

The M5 worldvolume theory can have global anomalies [105,40], related to the FreedWitten D-brane anomalies [46]. The anomaly analysis for D-branes in orientifolds is quite subtle and does not follow directly from the results of 46. A systematic analysis has not yet been carried out in the literature 10 From the M5-brane point of view it is clear though that there are no anomalies in the case at hand. The M5 analog [105] of the half integer shift in the quantization of the D-brane gauge field strength [46] is absent because $H^{3}(M 5, \mathbb{Z})=0$. The torsion anomaly [105,40], which is the analog of the $W_{3}=H$ anomaly for D-branes, vanishes as well. One way to see this is that when $H^{3}(M 5, \mathbb{Z})=0$, the function $\Omega: H^{3} \rightarrow \mathbb{Z}_{2}$ of $[105]$ is trivial, and therefore the anomaly constraint (5.10) of [105] becomes $\left.G\right|_{M 5}=0$ (in integral cohomology), whose de Rham part is just the usual tadpole cancellation condition for the M5 2-form field. This is clearly satisfied in our case as the only 4-fluxes we turn on are Poincaré dual to flat sliding Lagrangian cycles in the $\left(T^{2}\right)^{4} / \mathbb{Z}_{2}^{3}$ limit, and these manifestly have zero intersection with any of the divisors we are considering. An alternative and in some cases more accurate way of seeing the absence of this anomaly is by using the criterion in terms of the 8-cohomology class $\Theta_{X}$ of [40].

Next, consider the contributions from gaugino condensation. For brevity we will restrict to the symmetric resolution from now on. (It turns out that if one proceeds with the asymmetric resolution as we will do for the symmetric one in the following, no critical points of $W$ are found within the Kähler cone, or at least no simple ones, so we do not

9 We thank G. Moore for several discussions on these issues and on the topological constraints discussed below.

10 Note added: we were informed by D. Freed and G. Moore that they obtained the general anomaly cancellation conditions for orientifolds [45], and that for our model, it appears that indeed the anomaly can be cancelled by turning on a suitable $B$-field $\sum_{i \alpha} D_{i \alpha} / 2 \bmod 1$. This value of $B$ also cancels the unwanted "anomalous" tadpole contributions, closely related to the Freed-Witten anomaly, which in general appear on non-spin D-branes [80]. 
lose much by making this restriction.) As explained in section 2, the 7-brane stacks wrap 12 disjoint rigid divisors of topology $\mathbb{P}^{1} \times \mathbb{P}^{1}$, and each stack consists of an O7 with four D7's on top, giving rise to an $S O(8)^{12}$ gauge theory without any light charged matter. The latter is true at least if no mobile D3-branes are close to the 7-branes and if no gauge flux is turned on in the D7-branes. But since we saturate the entire D3 tadpole with 3-form flux, and because nothing forces us to turn on gauge flux in the branes, 11 these provisos are indeed met.

Thus, for the symmetric resolution, in our normalization conventions, the dimensionless superpotential $\widetilde{W}$ (see appendix A) we consider is:

$$
\widetilde{W}=\widetilde{W}_{0}+\sum_{i<j, \alpha, \beta} b_{i \alpha, j \beta} e^{-2 \pi \tau_{i \alpha, j \beta}}+\sum_{i, \alpha} c_{i \alpha} e^{-2 \pi \tau_{i \alpha} / 6}
$$

where $\widetilde{W}_{0}$ is the flux contribution discussed in section 5 . There can be higher order terms, for example from multi-wrapped instantons. But we will see below that at the critical point, these can be neglected.

After integrating out the dilaton and complex structure moduli, $\widetilde{W}_{0}, b_{i \alpha, j \beta}$ and $c_{i, a \alpha}$ are constants. Because of the rich dependence of $W$ on the Kähler moduli, one expects critical points for generic values of these constants.

To verify this explicitly, let us first try to simplify the general form of $W$. First, of course, we can pick a Kähler gauge in which $\widetilde{W}_{0}$ is real and negative. Because of the symmetry between the divisors, 12 we can also assume that the absolute values of all D3 instanton and all gaugino condensation coefficients are equal, i.e. $\left|b_{i \alpha, j \beta}\right|=b$ and $\left|c_{i \alpha}\right|=c$. There could be phase differences, as long as the phases form a representation of the symmetry group. However, because the Kähler potential does not depend on the imaginary parts of the 51 coordinates $\tau_{a}$ (i.e. the axions), we can redefine these imaginary parts by shifts $\operatorname{Im} \tau_{a} \rightarrow \operatorname{Im} \tau_{a}+c_{a}$ together with compensating shifts of the phases of the coefficients without altering the critical point equations,, 13 and so we can effectively put the

11 In general, the Freed-Witten anomaly [46] may force turning on gauge flux on branes, but since $\mathbb{P}^{1} \times \mathbb{P}^{1}$ is spin, there is no anomaly here.

12 The complex structures could break this symmetry, but in the explicit examples given in the previous section, this is not the case.

13 In the presence of nonperturbative corrections to the Kähler potential, it is no longer true that the critical point equations remain invariant under phase shifts. We will show though that these corrections are small at the critical point. 
phases of any subset of 51 coefficients equal to 1 (keeping in mind the modified relations between $\operatorname{Im} \tau_{a}$ and the physical axions defined by the periods of $C_{4}$ ). This together with the symmetry is sufficient to make all 60 phases of the coefficients in (6.13) effectively equal.

A more detailed argument for the last assertion goes as follows. We can certainly set the 48 phases of the D3-instanton contributions to zero by shifting 48 axions, since all 48 exceptional divisors are linearly independent. Furthermore we can use the remaining 3 axion degrees of freedom to put the phase of one gaugino condensate in each of the 3 distinct groups of four to 1 . Given the $S L(2, \mathbb{Z})$ symmetry within a given group of four, of which the phases must furnish a representation, the four resulting phases in a group can be either all +1 or two -1 and two +1 . Now note that the phases of the $S O(8)$ gaugino condensates can still be shifted by sixth roots of unity. This corresponds to shifting the 51 axions by various multiples of $2 \pi$, which will not change the phases of the D3-instantons, but gives more than enough freedom to change any gaugino condensate phase by an arbitrary sixth root of unity. Since -1 is a sixth root of unity, we can use this to flip the remaining -1 phases to +1 . Thus we conclude that, after suitable shifts of the axion coordinates, we can take all $b_{i \alpha, j \beta}=b>0$ and $c_{i \alpha}=c>0$.

We don't know exact expressions for $b$ or $c$, but fortunately the stabilization problem is not very sensitive to the precise values, as long as they are larger than $W_{0}$. One could assume the coefficients to be essentially of order 1 in the Kähler gauge where $K_{0} \equiv 0$ (with $K_{0}$ as in (6.5) being the complex structure and dilaton contribution to the Kähler potential). A potential problem in general is that there could be suppression factors of the form $e^{-c / g_{s}}$ for the D3 instantons, but as discussed earlier, these are absent in our model. In fact, if one takes into account curvature corrections in the Dirac-Born-Infeld action for the D3 instantons, which induce a negative $(-1)$-brane charge $-\chi(D) / 24$ [55,28] and therefore by supersymmetry a negative contribution to the effective volume, there will be an enhancement of the coefficient by a factor

$$
b_{g_{s}} \sim e^{\frac{2 \pi \chi(D)}{24 g_{s}}} .
$$

At weak string coupling this quickly becomes very large. In the following we will not take this enhancement into account and just put $b \equiv c \equiv 1$, keeping in mind that this is likely to be a significant underestimate. Since larger coefficients give larger volumes (as $D W=0$ roughly sets $b e^{-2 \pi V_{D}} \sim \widetilde{W}_{0}$ ), this implies that the volumes at the critical point we will compute are likely to be significantly below their exact values. 
To summarize, in the Kähler gauge $K_{0} \equiv 0$, we will take our superpotential to be

$$
\widetilde{W}=\widetilde{W_{0}}+\sum_{i<j, \alpha, \beta} e^{-2 \pi \tau_{i \alpha, j \beta}}+\sum_{i, \alpha} e^{-2 \pi \tau_{i \alpha} / 6}
$$

with $\widetilde{W}_{0}<0$ given by the values of $-e^{K / 2}|W|$ in the notation of section 5 , and appropriate redefinitions of the coordinates $\tau_{a}$ understood to incorporate the required axion shifts.

\subsection{Critical points}

To simplify things, we will look for candidate critical points on the locus of moduli space maximally respecting the symmetries between the divisors. For the symmetric resolution this amounts to setting

$$
t_{i \alpha, j \beta} \equiv t, \quad r_{i} \equiv r
$$

Then the compactification volume, relevant divisor volumes, and areas of the Mori generators are given by

$$
\begin{aligned}
V & =r^{3}-24 r t^{2}+48 t^{3} \\
V_{i \alpha, j \beta}=V_{E} & =r t-3 t^{2} \\
V_{i \alpha}=V_{D} & =r^{2}-8 r t+16 t^{2} \\
A_{i, j \beta}=A_{r} & =r-4 t \\
A_{\alpha \beta \gamma}=A_{t} & =\frac{t}{2}
\end{aligned}
$$

To further simplify the problem, we will also restrict our search of vacua to the (shifted) axion-free case $\operatorname{Im} \tau_{a}=0.14$ Under these assumptions, the superpotential (6.14) becomes

$$
\widetilde{W}=\widetilde{W}_{0}+48 e^{-2 \pi\left(r t-3 t^{2}\right)}+12 e^{-2 \pi\left(r^{2}-8 r t+16 t^{2}\right) / 6}
$$

Supersymmetric vacua are given by Kähler covariant critical points of $W$, i.e. solutions to $D_{i} W \equiv \partial_{\tau_{i}} W+\left(\partial_{\tau_{i}} K\right) W=0$, or equivalently ordinary critical points of $e^{K / 2}|W|$. Clearly, $e^{K / 2}|W|$ expanded around $\operatorname{Im} \tau=0$ is quadratic in $\delta \operatorname{Im} \tau$, so the axion-free case is automatically critical in the axion directions.

14 Relaxing this would presumably provide a large number of additional vacua. 


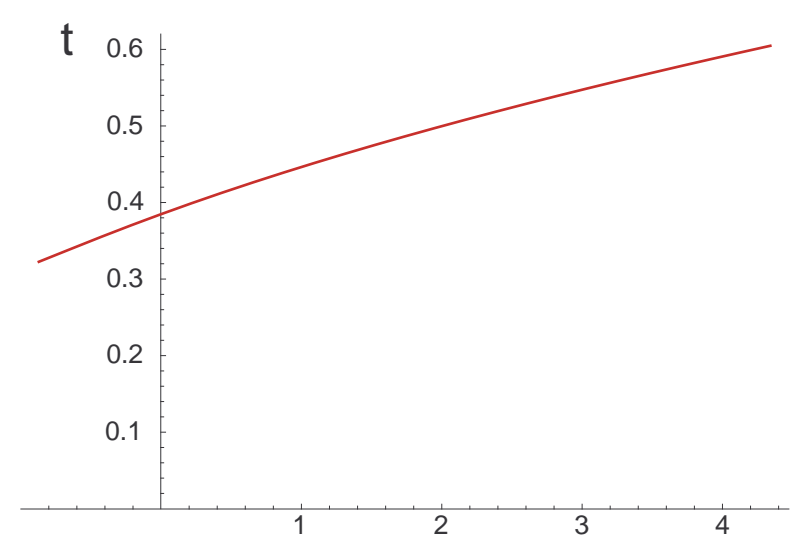

a
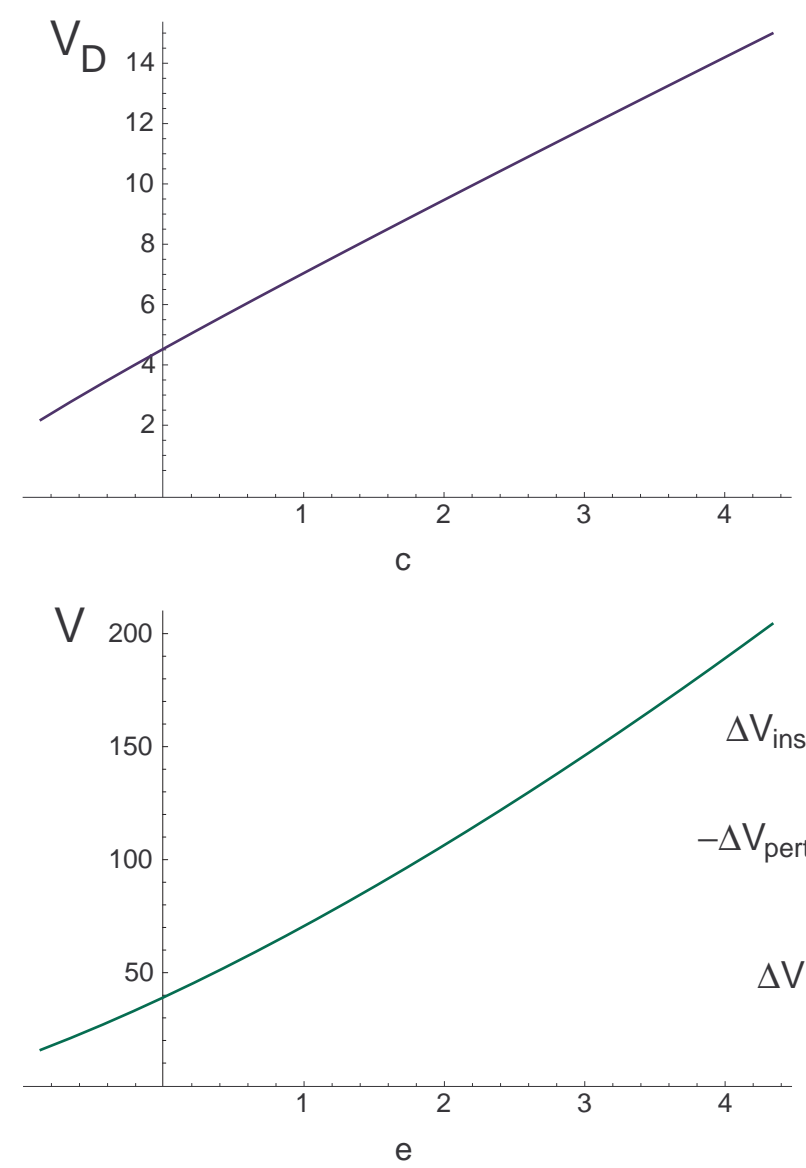

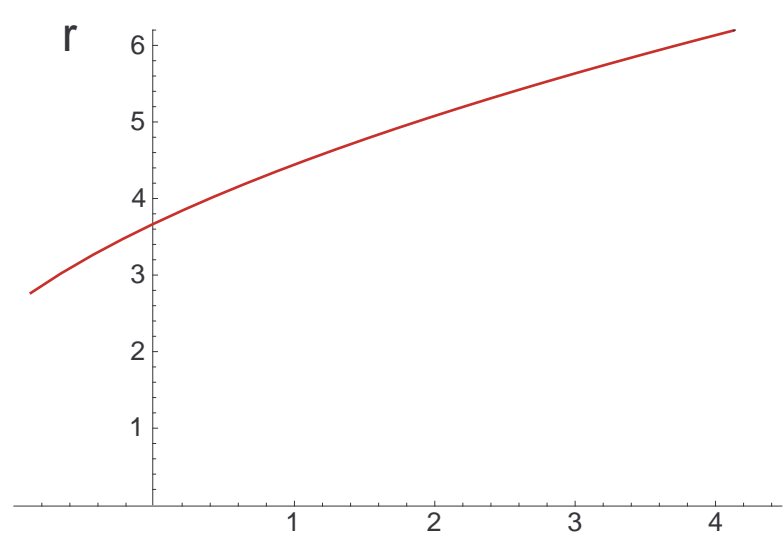

b
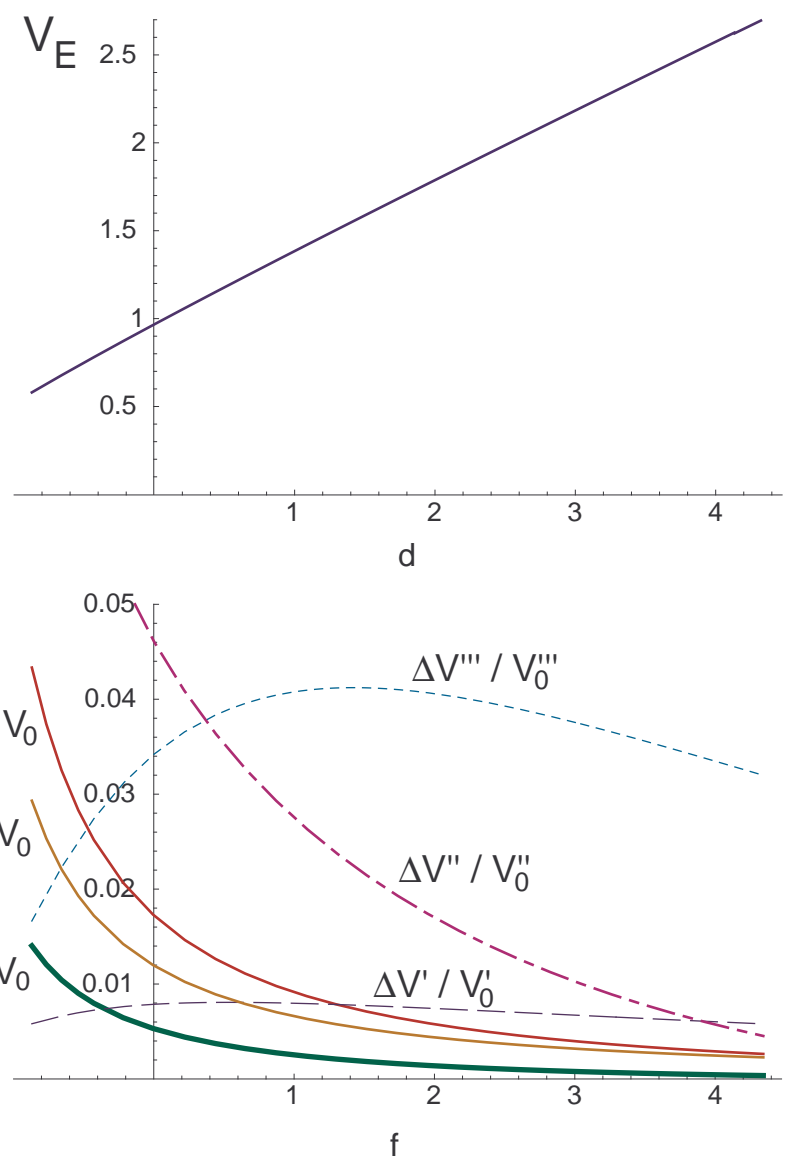

Fig. 19: Numerical results for critical point moduli as a function of the flux superpotential $\left|\widetilde{W}_{0}\right|$. The $x$-axis shows everywhere $-\log _{10}\left|\widetilde{W}_{0}\right|$ and the $y$-axis in (a): $t,(\mathrm{~b}): r,(\mathrm{c}): V_{D},(\mathrm{~d}): V_{E}$ and (e): $V$, as defined in (6.16). Finally (f) shows the estimated relative size of perturbative and instanton corrections to $V$ and its derivatives $\partial_{t}^{k} V, k=1,2,3$, for $g_{s} \sim 1 / 4$. 
The critical points of $e^{K / 2}|W|$ as a function of $r$ and $t$ are easily found numerically. The results for various values of $\widetilde{W}_{0}$ are shown in fig. 19 (a)-(e). Because the critical point equation roughly fixes $e^{-2 \pi V_{D} / 6} \sim e^{-2 \pi V_{E}} \sim \widetilde{W}_{0}$, the volume of a $2 n$-dimensional cycle grows as $\left(-\log \left|\widetilde{W}_{0}\right|\right)^{n / 2}$, which is clearly reflected by the figure. Note in particular the rather weak dependence on $\widetilde{W}_{0}$, allowing solutions with moderately large volumes up to $\widetilde{W}_{0} \sim 10$, which is the order of magnitude of the effective coefficients in (6.17). Similarly, as we verified numerically, there is only weak logarithmic dependence on the relative size of the coefficients of the exponentials.

For the explicit example A in section 5 , we have $\widetilde{W}_{0} \sim 0.3$, so

$$
r \approx 4, \quad t \approx 0.4
$$

and the various volumes in (6.16) are

$$
V \approx 55, \quad V_{E} \approx 1, \quad V_{D} \approx 6, \quad A_{r} \approx 2.5, \quad A_{t} \approx 0.2
$$

Recall that these are measured in units of $\ell_{s}=2 \pi \sqrt{\alpha^{\prime}}$. As discussed in the previous subsection, we actually expect the coefficients of the exponentials in $\widetilde{W}$ to be significantly larger than we assumed at weak string coupling. Since increasing these coefficients is equivalent to decreasing $\widetilde{W}_{0}$, this could easily give values of the volumes corresponding to an effective $\widetilde{W}_{0}$ a few orders of magnitude smaller than what we assumed.

Finally, we should still verify that the critical points we found are also critical for variations away from the ansatz (6.15), but because of the symmetry between the divisors, this turns out to be automatic. We checked this explicitly numerically by computing the full $D W$ at the candidate critical point. We also checked that there are no flat directions by computing the full $51 \times 51$ mass matrix. In fact, we found that the supergravity potential is at a local minimum at this critical point in all directions.

\subsection{Corrections}

There may be higher order nonperturbative corrections to the superpotential for example from multi-wrapped instantons, from more complicated divisors of holomorphic Euler characteristic 1, or even from divisors of holomorphic Euler characteristic $>1$ [53]. Because the divisors we took into account form a basis (in the sense that all other effective divisors are linear combinations of those with positive coefficients, and in particular have 
larger volumes), these will be suppressed by positive integral powers of the original exponentials. For the values of (6.19), we get $e^{-2 \pi V_{E}} \sim 5 \times 10^{-4}$, and $e^{-2 \pi V_{D} / 6} \approx 2 \times 10^{-3}$. Therefore these higher order contributions can reasonably be expected to be negligible.

Because of the smallness of these exponentials, it also follows that the dependence of the coefficients $b, c$ on the dilaton and complex structure moduli will not significantly affect the critical point of the flux superpotential, as long as the relative derivatives such as $b^{\prime} / b$ and $b^{\prime \prime} / b$ are not huge [65]. This justifies integrating out these moduli first.

The Kähler potential receives perturbative and nonperturbative corrections. The leading order perturbative correction appears at order $\alpha^{\prime 3}$ and was derived in the orientifold context in [10]. In our conventions, i.e. defining $V=\operatorname{vol}(B)=\operatorname{vol}(Y) / 2$ in units of $\ell_{s} \equiv 2 \pi \sqrt{\alpha^{\prime}}$ and in Einstein frame:

$$
K=-2 \ln \left(V+\frac{\xi}{g_{s}^{3 / 2}}\right), \quad \xi \equiv-\frac{\chi(Y) \zeta(3)}{8(2 \pi)^{3}} \approx-0.06 .
$$

Here we used $\chi(Y)=2\left(h^{1,1}-h^{2,1}\right)=96$. Since in our example $V \sim 55$ and $g_{s} \sim 1 / 4$, this correction is at the $1 \%$ level and therefore negligible (see fig. 19 (f) for more general values of $\widetilde{W}_{0}$ ). This remains true as long as $g_{s} \gg 1 / 100$.

Finally, there will also be instanton corrections to the Kähler potential. The most important corrections come from fundamental string worldsheet instantons, whose amplitude (in Einstein frame) is proportional to

$$
\Delta K \sim e^{-2 \pi \sqrt{g_{s}} A},
$$

with $A$ a holomorphic curve area on the covering Calabi-Yau space $Y$, expressed in terms of the areas of the Mori generators of the quotient $B$ as $A=m A_{r}+2 n A_{t}, m, n>0$. The factor of 2 is due to the fact that the area of the exceptional curves is twice as large in the covering space than in the quotient. That this is the appropriate area rather than the quotiented one was explained in section 2. (At any rate, this does not qualitatively alter the conclusions.)

Since $e^{-2 \pi A_{r}} \sim 10^{-7}$ and $e^{-4 \pi A_{t}} \sim 0.1$ for the values of (6.19), it is reasonable (at least for $g_{s}$ not too small) to assume that the main contribution will come from instantons with $m=0$, i.e. worldsheets wrapping the exceptional curves. To estimate their contribution, we use the multicover formula for the corresponding corrections to the $\mathcal{N}=2$ prepotential 
$\mathcal{F}$ (of which the leading part is essentially the string frame volume of $Y$, i.e. $\mathcal{F} \sim 2 g_{s}^{3 / 2} V$ ) 61]:

$$
\Delta \mathcal{F}=\frac{1}{(2 \pi)^{3}} \sum_{n=1}^{\infty} \frac{1}{n^{3}} e^{-2 \pi \sqrt{g_{s}}\left(2 A_{t}\right) n} \approx 10^{-3} .
$$

The factor $1 /(2 \pi)^{3}$ can be understood from the fact that the third derivative of the prepotential should give a $q$-expansion with coefficients equal to the Gromov-Witten invariants. The prefactor of the exponentials is 1 because the exceptional curves are isolated and unique. There are $64 \times 3=192$ such minimal exceptional curves, so for the example this gives a total contribution at the one percent level compared to $\mathcal{F}=2 g_{s}^{3 / 2} V \sim 14$. Similarly the relative corrections to the first three $t$-derivatives of $\mathcal{F}$ are at most at the few percent level in the entire range of interest.

Actually the above estimate is a bit imprecise, in that the $\mathcal{N}=2$ type II relation between $e^{-K}$ and $\mathcal{F}$ is somewhat more complicated in the presence of corrections, and that there are also contributions from curves wrapping the sums of two and three exceptional curves associated to the same triple intersection point (with Gromov-Witten invariants -1 resp. +1$)$. It is not hard though to make a more precise estimate taking this into account. The resulting relative correction is very close to the less refined estimate given above at the values of example (but unlike the less refined estimate is actually analytic in $t$ at $t=0$ ). We used this more precise estimate in our numerical computations for fig. 19(f).

These estimates indicate that even for our explicit examples with modest values of $\widetilde{W}_{0}, \alpha^{\prime}$ corrections are small.

One could also worry about tensionless strings appearing, for example from D3-branes wrapping the exceptional curves. However, since in the range of parameters of interest these curves all have string scale area, the effective tension of such strings will be comparable to that of a fundamental string.

Less is known about string loop corrections, but there is no reason to believe that these will destabilize the solution for the values of $g_{s}$ we are considering, and since anyway the number of flux vacua with $g_{s}$ less than $g_{*}$ scales only linearly with $g_{*}$ [35], there should be many more flux vacua with significantly smaller $g_{s}$ once we generalize the ansatz of section 5 to allow the $\tau_{i}$ to vary independently.

Thus we conclude that all evidence points towards the existence of a supersymmetric minimum near the value we obtained.

One could also argue more generally that a critical point of the supergravity potential should exist. It is straightforward to verify that this potential approaches zero from below 
in the limit of large radii and small $g_{s}$, where we trust the leading approximation. Therefore, assuming the supergravity potential stays bounded below in the interior of moduli space, a critical point should exist (although in general it may be nonsupersymmetric).

\section{Models with a single Kähler parameter}

There has been some confusion in the literature about the status of KKLT models with a single Kähler parameter [37,88]. Here we show that there exist models for which it is possible in principle to stabilize the single Kähler parameter.

We start with $\pi: Z \rightarrow B$ the elliptic fibration over the base $B=\mathbb{P}^{3}$. The corresponding Weierstrass model $W$ is given by the following hypersurface in $\mathbb{P}\left(\mathcal{O}_{B} \oplus \mathcal{O}_{B}\left(-2 K_{B}\right) \oplus\right.$ $\left.\mathcal{O}_{B}\left(-3 K_{B}\right)\right)$

$$
y^{2}=x^{3}+x f+g
$$

where $f \in H^{0}\left(B,-4 K_{B}\right)$ and $g \in H^{0}\left(B,-6 K_{B}\right)$. The pullback of the hyperplane divisor in the base has holomorphic Euler characteristic $\chi_{h}\left(\pi^{*} D\right)=-2$, and there is no nonperturbative superpotential generated by threebrane instantons. We can now enforce ADE type singularities of the Weierstrass model along a hyperplane divisor in the base, say $D_{1}$ given by $z_{1}=0$, where $\left(z_{1}: z_{2}: z_{3}: z_{4}\right)$ are homogeneous coordinates on $B$. In general, there exists a crepant resolution $\pi: \widetilde{Z} \rightarrow W$ of the Weierstrass model and the exceptional divisors contracted by $\pi$ have holomorphic Euler characteristic 1.

As an example, we consider a Weierstrass model where we enforce $I_{3}$ fibers (corresponding to an $A_{2}$ singularity) along the divisor $D_{1}$. This model has a toric crepant resolution; the vertices of the $\nabla$ polyhedron are given by

$$
\begin{aligned}
& (1,0,0,2,3),(1,0,0,1,2),(1,0,0,1,1),(0,1,0,2,3),(0,0,1,2,3),(-1,-1,-1,2,3) \\
& (0,0,0,-1,0),(0,0,0,0,-1)
\end{aligned}
$$

The first three divisors are vertical and of holomorphic Euler characteristic 1 . They are elliptically fibered over $D_{1} \simeq \mathbb{P}^{2}$. However, $D_{1}$ is not spin, but rather spin ${ }^{\mathrm{c}}$. Therefore, in order to cancel the Freed-Witten anomaly [46], we need to turn on a half-integrally quantized flux on the worldvolume of the D3 brane that wraps the divisor 15 . The requirement that this flux satisfies the DUY equations can then be satisfied only if the classical volume of the divisor vanishes.

15 We thank S. Sethi for a discussion on this point. 
However, there are other 1-parameter threefolds whose anticanonical divisor is very ample. Over these threefolds there exists a smooth Weierstrass model and we can therefore repeat the above analysis. The only requirement is that the hyperplane divisor in the base is spin. It is easy to see that the smooth quadric hypersurface $Q \subset \mathbb{P}^{4}$ has this property. Therefore, for this model it is possible in principle to generate a nonperturbative superpotential for the single Kähler modulus and stabilize it at moderately large values, provided that turning on fluxes fixes the complex structure moduli of the Weierstrass model at the singular locus. The F-theory compactification on this manifold was described in [50].

\section{Discussion}

We showed that with existing techniques, one can go a very long way towards demonstrating the existence of string vacua with all moduli fixed by a combination of fluxes and nonperturbative effects, by considering a particular model, explicitly computing the effective potential and finding its minima.

A next step in developing a proof would be to compute the D3-instanton prefactors in section 6. As discussed there these are expected to be $O(1)$, they are clearly $O(1)$ for the corrections with a gauge theory interpretation, and the construction will work for a wide range of $O(1)$ values. While it would be surprising if there were a problem at this stage, one would need explicit results to eventually prove that subleading corrections can be neglected.

Indeed, while one might think that proving the existence of vacua would require computing the exact scalar potential, all known corrections to the effects we used can be estimated by analogy to those found in simpler models, and we were able to argue that they would spoil the stability of our vacua only if they were far smaller or larger than expected. Thus, a proof might only require establishing rather weak bounds on the corrections.

Here we carried out the construction for a supersymmetric AdS ground state. It is also quite easy to construct $\mathrm{dS}$ vacua in our model at the four dimensional effective field theory level, by adding a number of anti-D3 branes and minimizing the total classical potential thus obtained. For suitable values of $W_{0}$, this will give a local minimum with positive energy. Unfortunately, in our model, with no large warp factors and in which $W_{0}$ is not so small, this would break supersymmetry near the string scale, so there would be no small parameter suppressing further corrections to the potential, and these vacua would not be 
trustworthy. However, in similar examples, one could in principle establish the existence of better controlled dS vacua at this level with existing techniques. Besides studying models with a conifold singularity and adding anti-D3 branes in the warped region [68,50,63, , one could also gain extra positive energy at the minimum by using critical points of the flux potential with a non-vanishing IASD component for the three-form flux [89]. Since such vacua are generic and their number scales like $F^{6}$ [36,41], the number of such dS vacua with small enough F-terms to admit parametric control is expected to be very large, although such models may be a small fraction of all candidate vacua.

The model discussed here has several advantages which made a concrete treatment possible, in particular it starts with 55 massless fields, many fewer than the $O(1000)$ typical of $\mathcal{N}=1$ models with many gauge groups, and these are further related by known discrete symmetries. Furthermore, since $b_{3}$ was small, and the special geometry for this $\mathrm{CY}$ is inherited from that of $T^{6}$, we could easily find explicit flux vacua. However none of this seems essential, nor did any other features clearly particular to this model enter explicitly into our discussion.

What must be clearly distinguished are the technical difficulties of the problem of exhibiting explicit vacua, from the actual constraints which determine their number. Even the best posed part of the problem, finding explicit supersymmetric flux vacua with small $W_{0}$, is very challenging. First, setting up the problem by solving the Picard-Fuchs equations and finding the explicit change of basis to a symplectic integral basis for $H^{3}$ in problems with hundreds of moduli is highly nontrivial. Then, since the number of flux vacua grows exponentially with $b_{3}$, listing them explicitly or even finding those with small $W_{0}$ is computationally difficult. While the study of Kähler stabilization is still in its early stages, it is very optimistic to think that once that is understood it will be easier; one expects this will be of comparable difficulty.

What could save the situation to some extent is the general idea that the problem of stabilizing moduli is largely a problem of satisfying many nearly independent conditions, which can be studied separately, as illustrated by the explicit constructions here and in [37]. For example, we have seen that if one turns on only three-form fluxes in the IIB compactification manifold (and e.g. no field strengths on various branes), the problem of stabilizing Kähler moduli can be dealt with almost independently of the flux stabilization of complex structure moduli; the two problems talk to each other, at leading order, only through the existence of the constant $W_{0}$ generated by the flux potential. Similarly, the problem of stabilizing Kähler moduli depends on the existence of a suitably large set 
of instanton corrections, but the existence of one instanton correction does not directly influence the existence of another.

This rough independence between the conditions is what makes a statistical approach reasonable, in which one estimates the fraction of vacua satisfying each condition, and multiplies these estimates to estimate the total fraction of stable vacua [43. Indeed, this approach appears far more widely applicable; for example in finding flux vacua, while the many fluxes and complex structure moduli do interact, to a good approximation the different components of the defining equations ( $D W=0$ for supersymmetric vacua) can be treated independently at each point in moduli space, to estimate the total number of flux vacua [6]. The general principle is simply that most of the many choices and structures in the problem are not directly relevant to the final outcome (stabilizing moduli, or finding other observables) and can with due care be approximated or left out to get these estimates.

In this spirit, the present results provide a start for evaluating the general claim that stabilized vacua are common, by making the recipe of [65] sufficiently concrete that it could be tried in a large list of models. The work so far [37,98] suggests that stabilizing Kähler moduli is generic, but this remains to be verified.

The model we studied actually has many stringy avatars, and was identified early in the duality revolution as one of the simplest fourfolds with many dual descriptions [52]. It could be worthwhile to flesh out the physics of our model from suitable heterotic or type I dual descriptions; perhaps by combining the insights from various dual pictures, one can obtain a better understanding of the physics of such stabilized models. These models also provide simple laboratories where the growing knowledge about flux-induced soft-breaking terms in D3-D7 systems [25, 58, 62, 74, 76] can be combined with full moduli stabilization to study how the spectrum of soft masses is modified. In addition, close relatives of this model (which exist at different discrete choices of the $B$-field in the orbifold singularities) have played an important role in constructions of semi-realistic particle physics models $19,20,27,30,44,75,78$, and such constructions may also be possible in simple extensions of our setup.

Another recent suggestion for stabilizing F-theory models with several Kähler moduli in a controllable regime, using the same ingredients as in [65] but in a different scaling regime, appeared in [8,9]; it would be interesting to see if similarly explicit constructions are possible in that context.

For the AdS vacua presented here, it would be very interesting to proceed along the lines of [94] and try to determine a dual CFT. Since we only use a few fluxes, the brane 
constructions invoked in [94] may be amenable to explicit study. However, it is important to keep in mind that for theories of this sort where the geometrical or gravitational picture is under reasonable control, one expects the CFT to be strongly coupled. We have no powerful techniques for studying isolated, strongly coupled conformal fixed points, and so far such dualities have mostly been useful in using the gravity picture to learn about the CFT, as in [94.

The existence of the landscape would seem to require there to be a huge set of such $3 D$ CFTs. Unlike the classes of vacua which are constructed by a Freund-Rubin ansatz using a single $p$-form flux threading a $p$-sphere, which are prominent in the simplest AdS/CFT dualities, the IIB flux vacua involve two different three-forms threading many cycles of the compact geometry. For this simple reason, the degeneracy of expected vacua is much larger than in the most familiar examples of the Freund-Rubin ansatz.

We also note that in contrast to the simple examples of Freund-Rubin vacua, in our constructions the compact dimensions can be parametrically small compared to the curvature scale of the noncompact dimensions (which is of course required for any model of phenomenological interest). In particular, there is no KK tower of modes with masses given by the inverse AdS radius - while in un-tuned constructions the lightest moduli will have masses in this range, they do not arise as the lowest modes in infinite towers. This means that the $3 D$ CFTs which will arise are rather different than e.g. the theory on $N$ M2 branes at large $N$. This is not a surprise; the properties of weakly coupled theories are always astonishing when viewed from the strongly coupled side of a dual pair. In particular, the properties of weakly coupled or even free field theory seem truly amazing as predictions about strongly coupled, highly curved gravitational backgrounds! Similarly, here the more controlled side of the duality involves the compactification geometry, and the properties of the strongly coupled dual field theories are likely to be interesting.

\section{Acknowledgements}

We are grateful to P. Aspinwall, D.-E. Diaconescu, A. Giryavets and especially G. Moore for many helpful discussions. We also benefited from discussions with N. Berkovits, O. DeWolfe, C. Florea, R. Kallosh, S. Katz, S. Sethi, E. Silverstein, W. Taylor, S. Trivedi and J. Walcher. The work of F.D., M.R.D. and B.F. is supported in part by DOE grant DE-FG02-96ER40949. S.K. was supported in part by a David and Lucile Packard Foundation Fellowship for Science and Engineering, the NSF under grant PHY-0244728, and the DOE under contract DE-AC02-76SF00515. 


\section{Appendix A. Normalizations}

In this appendix we give our normalization conventions, and give expressions for the superpotential and Kähler potential adapted to the standard $\mathcal{N}=1$ four dimensional supergravity framework, where the four dimensional Planck mass $m_{p}$ is the only dimensionful constant appearing explicitly.

We define the string length $\ell_{s}$ as

$$
\ell_{s} \equiv 2 \pi \sqrt{\alpha^{\prime}}
$$

Then we have in the conventions of [87]:

$$
\frac{1}{2 \kappa_{10}^{2}}=\frac{2 \pi}{\ell_{s}^{8}}, \quad \mu_{p}=\frac{2 \pi}{\ell_{s}^{p+1}}, \quad T_{p}=\frac{\mu_{p}}{g_{s}}
$$

and

$$
\widetilde{F}_{k} \equiv \frac{1}{\ell_{s}^{k-1}} F_{k} \in H^{k}(Y, \mathbb{Z})
$$

We want to write the four dimensional effective action in the standard $\mathcal{N}=1$ supergravity form appropriate for dimensionless scalars, with $W$ of mass dimension 3 and the only dimensionful parameter appearing the four dimensional Planck mass $m_{p}$ :

$$
S=\int \frac{m_{p}^{2}}{2} R-\frac{1}{m_{p}^{2}} e^{K}\left(|D W|^{2}-3|W|^{2}\right)+\cdots
$$

The relation between $m_{p}$ and $\ell_{s}$ is given by

$$
m_{p}^{2}=\frac{4 \pi}{\ell_{s}^{2}} \frac{V_{s}}{g_{s}^{2}}=\frac{4 \pi}{\ell_{s}^{2}} \frac{V_{e}}{g_{s}^{1 / 2}},
$$

where $V_{s}$ is the string frame compactification volume in units of $\ell_{s}$ and $V_{e}$ the ten dimensional Einstein frame volume in units of $\ell_{s}$. In the bulk of the paper we work in Einstein frame (more precisely we rescale the metric of the compactification manifold with $g_{s}^{-1 / 2}$ ). Note that this absorbs for example a factor $1 / g_{s}$ in the action of a D3-brane instanton wrapping a divisor $D$ :

$$
S_{\text {inst }}=\frac{2 \pi}{g_{s}} V_{s}(D)=2 \pi V_{e}(D)
$$

where $V(D)$ denotes the volume of $D$ in units of $\ell_{s}$. The following Kähler potential and flux superpotential give the correctly normalized four dimensional action:

$$
K=-\ln (-i(\tau-\bar{\tau}))-\ln i \int \Omega \wedge \bar{\Omega}-2 \ln V_{e}
$$


and

$$
W_{\text {flux }}=\frac{m_{p}^{3}}{\sqrt{4 \pi}} \widetilde{W}_{\text {flux }} ; \quad \widetilde{W}_{\text {flux }} \equiv \int\left(\widetilde{F}_{3}-\tau \widetilde{H}_{3}\right) \wedge \Omega .
$$

This is written in such way that $W$ has mass dimension 3 , and such that only $m_{p}$ appears as a dimensionful parameter, as it should in an effective four dimensional theory. To avoid dragging along the prefactor in $W_{\text {flux }}$, we use the dimensionless superpotential $\widetilde{W}_{\text {flux }}$ in the bulk of this paper.

To verify the correctness of the normalization, it is sufficient to check the tension of a BPS domain wall between two flux vacua, say with different RR flux $\widetilde{F}_{3}$. In the four dimensional effective theory this is

$$
T=2 e^{K / 2}|\Delta W|=\frac{2 m_{p}^{3}}{\sqrt{4 \pi} \sqrt{2 / g_{s}} V_{e}} \frac{\left|\int \Delta \widetilde{F}_{3} \wedge \Omega\right|}{\left(\int i \Omega \wedge \bar{\Omega}\right)^{1 / 2}}
$$

In string theory the BPS domain wall can be thought of as a D5-brane wrapped around a special Lagrangian submanifold $L$ Poincaré dual to $\Delta \widetilde{F}_{3}$ (so $\int \Delta \widetilde{F}_{3} \wedge \Omega=\int_{L} \Omega$ ). It can be shown [17] that for a special Lagrangian $L$,

$$
\frac{\left|\int_{L} \Omega\right|}{\left(\int i \Omega \wedge \bar{\Omega}\right)^{1 / 2}}=\frac{V_{s}(L)}{\sqrt{8 V_{s}}}
$$

where $V_{s}(L)$ is the string frame volume of $L$ in units of $\ell_{s}$. Plugging this in (A.2) and using (A.1) gives

$$
T=\frac{2 \pi V_{s}(L)}{g_{s} \ell_{s}^{3}}=T_{5} \operatorname{Vol}_{s}(L),
$$

which agrees with the BPS domain wall tension obtained in the D-brane picture, confirming the correctness of our normalizations including all prefactors. 


\section{References}

[1] B. S. Acharya, "A Moduli Fixing Mechanism in M-Theory," hep-th/0212294.

[2] O. Aharony, C. Sonnenschein, S. Yankielowicz and S. Theisen, "Field Theory Questions for String Theory Answers", Nucl. Phys. B493 (1997) 177, hep-th/9611222.

[3] G. Aldazabal, A. Font, L. E. Ibanez and G. Violero, " $D=4, \mathcal{N}=1$, Type IIB Orientifolds," Nucl. Phys. B536 (1998) 29, hep-th/9804026.

[4] C. Angelantonj, I. Antoniadis, G. D'Appollonio, E. Dudas and A. Sagnotti, "Type I Vacua with Brane Supersymmetry Breaking," Nucl. Phys. B572 (2000) 36, hepth/9911081.

[5] C. Angelantonj and A. Sagnotti, "Open Strings," Phys. Rept. 371 (2002) 1, [Erratumibid. 376 (2003) 339], hep-th/0204089.

[6] S. Ashok and M. R. Douglas, "Counting Flux Vacua," JHEP 0401 (2004) 060, hepth/0307049.

[7] P. S. Aspinwall, "Enhanced Gauge Symmetries and K3 Surfaces," Phys. Lett. B357 (1995) 329, hep-th/9507012.

[8] V. Balasubramanian and P. Berglund, "Stringy Corrections to Kähler Potentials, Susy Breaking, and the Cosmological Constant Problem," JHEP 0411 (2004) 085, hepth/0408054.

[9] V. Balasubramanian, P. Berglund, J. Conlon and F. Quevedo, "Systematics of Moduli Stabilisation in Calabi-Yau Flux Compactifications," hep-th/0502058.

[10] K. Becker, M. Becker, M. Haack and J. Louis, "Supersymmetry Breaking and $\alpha^{\prime}$ Corrections to Flux Induced Potentials," JHEP 0206, (2002) 060, hep-th/0204254.

[11] O. Bergman, E. G. Gimon and S. Sugimoto, "Orientifolds, RR torsion, and K-theory," JHEP 0105 (2001) 047, hep-th/0103183.

[12] M. Berkooz and R. G. Leigh, "A $D=4 \mathcal{N}=1$ Orbifold of Type I Strings", Nucl. Phys. B483 (1997) 187, hep-th/9605049.

[13] M. Berkooz, R. G. Leigh, J. Polchinski, J. H. Schwarz, N. Seiberg and E. Witten, "Anomalies, Dualities, and Topology of $D=6 \mathcal{N}=1$ Superstring Vacua," Nucl. Phys. B475 (1996) 115, hep-th/9605184.

[14] N. Berkovits, "Quantum Consistency of the Superstring in $A d S_{5} \times S^{5}$ Background," hep-th/0411170.

[15] M. Bershadsky, K. Intriligator, S. Kachru, D. R. Morrison, V. Sadov and C. Vafa, "Geometric Singularities and Enhanced Gauge Symmetries", Nucl. Phys. B481 (1996) 215 , hep-th/9605200.

[16] M. Bianchi, Ph.D. thesis, preprint ROM2F-92/13; A. Sagnotti, "Anomaly Cancellations and Open-String Theories", hep-th/9302099.

[17] M. Billó, S. Cacciatori, F. Denef, P. Fré, A. van Proeyen and D. Zanon, "The 0-Brane Action in a General $D=4$ Supergravity Background," Class. Quant. Grav. 16, (1999) 2335 , hep-th/9902100. 
[18] J. D. Blum and A. Zaffaroni, "An Orientifold from F-Theory", Phys. Lett. B387 (1996) 71 , hep-th/9607019.

[19] R. Blumenhagen, M. Cvetic, F. Marchesano and G. Shiu, "Chiral D-Brane Models with Frozen Open String Moduli," hep-th/0502095.

[20] R. Blumenhagen, D. Lüst and T. Taylor, "Moduli Stabilization in Chiral Type IIB Orientifold Models with Fluxes," Nucl. Phys. B663 (2003) 319, hep-th/0303016.

[21] C. Borcea, "K3 Surfaces with Involution and Mirror Pairs of Calabi-Yau Manifolds", "Mirror Symmetry II", (1997) 717.

[22] R. Bousso and J. Polchinski, "Quantization of Four-Form Fluxes and Dynamical Neutralization of the Cosmological Constant," JHEP 0006 (2000) 006, hep-th/0004134.

[23] I. Brunner and K. Hori, "Orientifolds and Mirror Symmetry," JHEP 0411 (2004) 005, hep-th/0303135.

[24] I. Brunner, K. Hori, K. Hosomichi and J. Walcher, "Orientifolds of Gepner Models," hep-th/0401137.

[25] P. Camara, L. Ibanez and A. Uranga, "Flux-Induced Susy-Breaking Soft Terms on D7-D3 Brane Systems," Nucl. Phys. B708 (2005) 268, hep-th/0408036;

P. Camara, L. Ibanez and A. Uranga, "Flux Induced Susy Breaking Soft Terms," Nucl. Phys. B689 (2004) 195, hep-th/0311241.

[26] P. Candelas and A. Font, "Duality Between the Webs of Heterotic and Type II Vacua", Nucl. Phys. B511 (1998) 295, hep-th/9603170.

[27] J. Cascales and A. Uranga, "Chiral 4D String Vacua with D-Branes and NS-NS and R-R Fluxes," JHEP 0305 (2003) 011, hep-th/0303024.

[28] Y. K. Cheung and Z. Yin, "Anomalies, Branes, and Currents," Nucl. Phys. B517 (1998) 69, hep-th/9710206.

[29] B. Craps and F. Roose, "(Non-)Anomalous D-Brane and O-Plane Couplings: The Normal Bundle," Phys. Lett. B450 (1999) 358, hep-th/9812149.

[30] M. Cvetic, T. Li and T. Liu, "Standard-like Models as Type IIB Flux Vacua," hepth/0501041.

[31] A. Dabholkar and J. Park, "A Note on Orientifolds and F-theory," Phys. Lett. B394 (1997) 302, hep-th/9607041.

[32] K. Dasgupta, D. P. Jatkar and S. Mukhi, "Gravitational Couplings and $\mathbb{Z}_{2}$ Orientifolds," Nucl. Phys. B523 (1998) 465, hep-th/9707224.

[33] K. Dasgupta, G. Rajesh and S. Sethi, "M Theory, Orientifolds and G-Flux," JHEP 9908 (1999) 023, hep-th/9908088.

[34] X. de la Ossa, B. Florea and H. Skarke, "D-Branes on Noncompact Calabi-Yau Manifolds: K-Theory and Monodromy," Nucl. Phys. B644 (2002) 170, hep-th/0104254.

[35] F. Denef and M. R. Douglas, "Distributions of Flux Vacua," JHEP 0405 (2004) 072, hep-th/0404116. 
[36] F. Denef and M. R. Douglas, "Distributions of Nonsupersymmetric Flux Vacua," hepth/0411183.

[37] F. Denef, M. R. Douglas and B. Florea, "Building a Better Racetrack," JHEP 0406 (2004) 034, hep-th/0404257.

[38] O. DeWolfe, A. Giryavets, S. Kachru and W. Taylor, "Enumerating Flux Vacua with Enhanced Symmetries," hep-th/0411061.

[39] D.-E. Diaconescu, M. R. Douglas and J. Gomis, "Fractional Branes and Wrapped Branes," JHEP 9802 (1998) 013, hep-th/9712230.

[40] D.-E. Diaconescu, D. Freed and G. W. Moore, "The M-Theory 3-Form and E 8 Gauge Theory", hep-th/0312069.

[41] M. Dine, D. O'Neil and Z. Sun, "Branches of the Landscape," hep-th/0501214.

[42] M. R. Douglas and G. W. Moore, "D-Branes, Quivers and ALE Instantons", hepth/9603167.

[43] M. R. Douglas, "The Statistics of String/M-Theory Vacua," JHEP 0305 (2003) 046, hep-th/0303194.

[44] A. Font and L. Ibanez, "SUSY-Breaking Soft Terms in a MSSM Magnetized D7-Brane Model," hep-th/0412150.

[45] D. Freed and G. Moore, unpublished.

[46] D. Freed and E. Witten, "Anomalies in String Theory with D-Branes", hepth/9907189.

[47] A. Frey and J. Polchinski, "N $\mathcal{N}=3$ Warped Compactifications," Phys. Rev. D65 (2002) 126009, hep-th/0201029.

[48] E. Gimon and J. Polchiski, "Consistency Conditions for Orientifolds and D-Manifolds", Phys. Rev. D54 (1996) 1667, hep-th/9601038.

[49] O. Ganor, "A Note on Zeroes of Superpotentials in F-Theory", Nucl. Phys. B499 (1997) 55. hep-th/9612077.

[50] S. Giddings, S. Kachru and J. Polchinski, "Hierarchies from Fluxes in String Compactifications," Phys. Rev. D66 (2002) 106006, hep-th/0105097.

[51] A. Giryavets, S. Kachru, P. Tripathy and S. Trivedi, "Flux Compactifications on Calabi-Yau Threefolds," JHEP 0404 (2004) 003, hep-th/0312104.

[52] R. Gopakumar and S. Mukhi, "Orbifold and Orientifold Compactifications of FTheory and M-theory to Six- Dimensions and Four-Dimensions", Nucl. Phys. B479 (1996) 260, hep-th/9607057.

[53] L. Görlich, S. Kachru, P. K. Tripathy and S. P. Trivedi, "Gaugino Condensation and Nonperturbative Superpotentials in Flux Compactifications," hep-th/0407130.

[54] A. Grassi, "Divisors on Elliptic Calabi-Yau 4-Folds and the Superpotential in FTheory - I", math.AG/9704008.

[55] M. B. Green, J. A. Harvey and G. W. Moore, "I-Brane Inflow and Anomalous Couplings on D-Branes," Class. Quant. Grav. 14, (1997) 47, hep-th/9605033. 
[56] B. R. Greene, "D-Brane Topology Changing Transitions," Nucl. Phys. B525 (1998) 284, hep-th/9711124.

[57] P. Griffiths and J. Harris, "Principles of Algebraic Geometry", J. Wiley \& Sons, 1978, New York.

[58] T. W. Grimm and J. Louis, "The Effective Action of $\mathcal{N}=1$ Calabi-Yau Orientifolds", hep-th/0403067.

[59] S. Gukov, C. Vafa and E. Witten, "CFT's from Calabi-Yau Fourfolds," Nucl. Phys. B584 (2000) 69, hep-th/9906070.

[60] A. Hanany and B. Kol, "On Orientifolds, Discrete Torsion, Branes and M-Theory," JHEP 0006 (2000) 013, arXiv:hep-th/0003025.

[61] S. Hosono, A. Klemm and S. Theisen, "Lectures on Mirror Symmetry," hepth/9403096.

[62] H. Jockers and J. Louis, "The Effective Action of D7-Branes in $\mathcal{N}=1$ Calabi-Yau Orientifolds," Nucl. Phys. B705 (2005) 167, hep-th/0409098;

H. Jockers and J. Louis, "D-Terms and F-Terms from D7-Brane Fluxes," hepth/0502059.

[63] S. Kachru, J. Pearson and H. Verlinde, "Brane/Flux Annihilation and the String Dual of a Nonsupersymmetric Field Theory," JHEP 0206 (2002) 021, hep-th/0112197.

[64] S. Kachru, M. Schulz and S. Trivedi, "Moduli Stabilization from Fluxes in a Simple IIB Orientifold," hep-th/0201028.

[65] S. Kachru, R. Kallosh, A. Linde and S. P. Trivedi, "de Sitter Vacua in String Theory", Phys. Rev. D68 (2003) 046005, hep-th/0301240.

[66] R. Kallosh and D. Sorokin, "Dirac Action on M5 and M2 Branes with Bulk Fluxes," hep-th/0501081.

[67] S. Katz and C. Vafa, "Geometric Engineering of $\mathcal{N}=1$ Quantum Field Theories," Nucl. Phys. B497, (1997) 196, hep-th/9611090.

[68] I. Klebanov and M. Strassler, "Supergravity and a Confining Gauge Theory: Duality Cascades and $\chi$ SB Resolution of Naked Singularities," JHEP 0008 (2000) 052, hepth/0007191.

[69] M. Klein and R. Rabadan, "Orientifolds with Discrete Torsion," JHEP 0007 (2000) 040, arXiv:hep-th/0002103.

[70] M. Klein and R. Rabadan, " $D=4, \mathcal{N}=1$ Orientifolds with Vector Structure," Nucl. Phys. B596 (2001) 197, hep-th/0007087.

[71] M. Klein and R. Rabadan, " $\mathbb{Z}_{N} \times \mathbb{Z}_{M}$ Orientifolds with and without Discrete Torsion," JHEP 0010 (2000) 049, hep-th/0008173.

[72] K. Kodaira, "On Compact Analytic Surfaces II", Annals of Math. 77 (1963) 3.

[73] J. Kollár and S. Mori, "Birational Geometry of Algebraic Varieties", Cambridge University Press, Cambridge, U.K., 134 (1998). 
[74] D. Lüst, S. Reffert and S. Stieberger, "Flux-Induced Soft Supersymmetry Breaking in Chiral Type IIB Orientifolds with D3 / D7-Branes", Nucl. Phys. B706 (2005) 3, hep-th/0406092.

[75] D. Lüst, S. Reffert and S. Stieberger, "MSSM with Soft Susy Breaking Terms from D7-Branes with Fluxes", hep-th/0410074.

[76] D. Lüst, P. Mayr, S. Reffert and S. Stieberger, "F-Theory Flux, Destabilization of Orientifolds and Soft Terms on D7-Branes", hep-th/0501139.

[77] A. Maloney, E. Silverstein and A. Strominger, "de Sitter Space in Noncritical String Theory," hep-th/0205316.

[78] F. Marchesano and G. Shiu, "Building MSSM Flux Vacua," JHEP 0411 (2004) 041, hep-th/0409132.

[79] P. Mayr, "On Supersymmetry Breaking in String Theory and its Realization in Brane Worlds," Nucl. Phys. B593 (2001) 99, hep-th/0003198.

[80] R. Minasian and G. W. Moore, "K-Theory and Ramond-Ramond Charge," JHEP 9711 (1997) 002, hep-th/9710230.

[81] G. W. Moore, "Les Houches Lectures on Strings and Arithmetic," hep-th/0401049.

[82] G. W. Moore, "Anomalies, Gauss Laws, and Page Charges in M-Theory," hepth/0409158.

[83] V. V. Nikulin, "Discrete Reflection Groups in Lobachevsky Spaces and Algebraic Surfaces", Proc. ICM, Berkeley, California (1986) 654.

[84] J. Park, R. Rabadan and A. M. Uranga, "Orientifolding the Conifold," Nucl. Phys. B570 (2000) 38, hep-th/9907086.

[85] M. Reid, "Canonical 3-Folds" Journées de Géométrie Algébrique d'Angers (1980) 273.

[86] J. Polchinski, "Tensors from K3 Orientifolds", Phys. Rev. D55 (1997) 6423, hepth/9606165.

[87] J. Polchinski, "String Theory" vol. I and II, Cambridge University Press (1998).

[88] D. Robbins and S. Sethi, "A Barren Landscape", hep-th/0405011.

[89] A. Saltman and E. Silverstein, "The Scaling of the No-Scale Potential and de Sitter Model Building," JHEP 0411 (2004) 066, hep-th/0402135.

[90] A. Sen, "F-theory and Orientifolds," Nucl. Phys. B475, (1996) 562, hep-th/9605150.

[91] A. Sen, "Orientifold Limit of F-Theory Vacua", Phys. Rev. D55 (1997) 7345, hepth/9702165.

[92] A. Sen and S. Sethi, "The Mirror Transform of Type I Vacua in Six Dimensions," Nucl.Phys. B499 (1997) 45-54; hep-th/9703157.

[93] S. Sethi, "A Relation Between $\mathcal{N}=8$ Gauge Theories in Three Dimensions," JHEP 9811 (1998) 003, hep-th/9809162.

[94] E. Silverstein, "AdS and dS Entropy from String Junctions or the Function of Junction Conjunctions," hep-th/0308175. 
[95] B. J. Stefanski, "Gravitational Couplings of D-Branes and O-Planes," Nucl. Phys. B 548(1999) 275, hep-th/9812088.

[96] L. Susskind, "The Anthropic Landscape of String Theory," hep-th/0302219.

[97] T. Taylor and C. Vafa, "RR Flux on Calabi-Yau and Partial Supersymmetry Breaking," Phys. Lett. B474 (2000) 130, hep-th/9912152.

[98] P. Tripathy and S. Trivedi, "D3 Brane Action and Fermion Zero Modes in Presence of Background Flux," hep-th/0503072.

[99] C. Vafa and E. Witten, "On Orbifolds with Discrete Torsion," J. Geom. Phys. 15, 189 (1995) hep-th/9409188.

[100] C. Voisin, "Mirrors and Involutions on K3 Surfaces", Journées de Géométrie Algébrique d'Orsay, Astérisque 218 (1993) 273.

[101] E. Witten, "Non-Perturbative Superpotentials in String Theory", Nucl. Phys. B474 (1996) 343, hep-th/9604030.

[102] E. Witten, "On Flux Quantization in M-Theory and the Effective Action," J. Geom. Phys. 22 (1997) 1, hep-th/9609122.

[103] E. Witten, "Fivebrane Effective Action in M-Theory," J. Geom. Phys. 22 (1997) 103, hep-th/9610234.

[104] E. Witten, "Baryons and Branes in Anti de Sitter Space," JHEP 9807 (1998) 006, hep-th/9805112.

[105] E. Witten, "Duality Relations Among Topological Effects in String Theory", JHEP 0005 (2000) 031, hep-th/9912086. 